\title{
WestVirginiaUniversity
}

THE RESEARCH REPOSITORY @ WVU

Graduate Theses, Dissertations, and Problem Reports

2013

\section{Chalcopyrite Semiconductors as Sources for Terahertz Spectroscopy}

Joseph D. Rowley

West Virginia University

Follow this and additional works at: https://researchrepository.wvu.edu/etd

\section{Recommended Citation}

Rowley, Joseph D., "Chalcopyrite Semiconductors as Sources for Terahertz Spectroscopy" (2013).

Graduate Theses, Dissertations, and Problem Reports. 3663.

https://researchrepository.wvu.edu/etd/3663

This Dissertation is protected by copyright and/or related rights. It has been brought to you by the The Research Repository @ WVU with permission from the rights-holder(s). You are free to use this Dissertation in any way that is permitted by the copyright and related rights legislation that applies to your use. For other uses you must obtain permission from the rights-holder(s) directly, unless additional rights are indicated by a Creative Commons license in the record and/ or on the work itself. This Dissertation has been accepted for inclusion in WVU Graduate Theses, Dissertations, and Problem Reports collection by an authorized administrator of The Research Repository @ WVU.

For more information, please contact researchrepository@mail.wvu.edu. 


\title{
Chalcopyrite Semiconductors as Sources for Terahertz Spectroscopy
}

\author{
Joseph D. Rowley \\ Dissertation submitted to the \\ Eberly College of Arts and Sciences \\ at West Virginia University \\ in partial fulfillment of requirements \\ for the degree of
}

Doctor of Philosophy

in

Physics

\begin{abstract}
Alan D. Bristow, Ph. D., Chair
Paul A. Cassak, Ph. D.

Edward Flagg, Ph. D.

David Lederman, Ph. D.

Nianqiang $\mathrm{Wu}, \mathrm{Ph}$. D.

Department of Physics

Morgantown, West Virginia

2013
\end{abstract}

Keywords: nonlinear optics; ultrafast optics; optical rectification; broadband; terahertz; zinc germanium phosphide; chalcopyrite semiconductor; phase matching; birefringence; cadmium germanium phosphide 


\title{
Abstract \\ Chalcopyrite Semiconductors as Sources for Terahertz Spectroscopy
}

\author{
Joseph D. Rowley
}

This dissertation summarizes a first effort to address the suitability of chalcopyrite structured semiconductors for efficient and broadband terahertz ( $\mathrm{THz}$ ) pulse emission by optical rectification. The experiments demonstrate that $\mathrm{ZnGeP}_{2}$, a chalcopyrite semiconductor showing improved growth quality in recent decades [1,2], is a promising source for $\mathrm{THz}$ spectroscopy while also addressing general fundamental questions concerning the nonlinear generation process in chalcopyrite semiconductors. Beyond $\mathrm{ZnGeP}_{2}$, the potential of other chalcopyrite structured semiconductors for $\mathrm{THz}$ emission is examined in the context of calculated phase-matching characteristics, onset pump wavelength of multiphoton absorption, and tabulated second-order nonlinear coefficients. Based on these, $\mathrm{CdGeP}_{2}$ is determined to show particular promise among semiconductors as a $\mathrm{THz}$ source when pumped at or near the fiber laser line of $1.55 \mu \mathrm{m}$. The orientation dependence of $\mathrm{THz}$ optical rectification efficiency is examined in tetragonal birefringent $\mathrm{ZnGeP}_{2}$, compared to that of cubic GaP, and modeled based on the second-order nonlinear tensor, while accounting for birefringence. These results demonstrate the significant effects of birefringence on $\mathrm{THz}$ emission efficiency while defining the most efficient orientations for generation and can be generalized to other chalcopyrite semiconductors; the general approach is applicable to other uniaxial birefringent crystals. Experimental mapping of generated $\mathrm{THz}$ pulses is presented over a broad pump tuning range $(1120-2480 \mathrm{~nm})$ in $3 \mathrm{~mm}$ thick $\mathrm{ZnGeP}_{2}$ crystals. This mapping, for two distinct and efficient orientations, demonstrates the moderate angle tunability of phase mismatch achievable in chalcopyrite crystals; the data also continuously demonstrate the effects of phase mismatch on the temporal and spectral THz pulse waveform. Finally, pump-intensity dependence of emission is presented for three crystal lengths, showing the effects of near-infrared nonlinear optical absorption and $\mathrm{THz}$ free-carrier absorption. Measurements, modeling, and calculation of characteristic lengths provide insight into the upper limit of pulse intensity and crystal length for generation of intense terahertz pulses without detriment to the bandwidth. Direct comparison of generation efficiency from $\mathrm{GaP}$ and $\mathrm{ZnGeP}_{2}$ is also measured for reference. 


\section{Acknowledgements}

I agree with the sentiment of Isaac Asimov who said, "The saddest aspect of life right now is that science gathers knowledge faster than society gathers wisdom." It's only fitting then that some wisdom be added to the small contribution to scientific knowledge summarized in this dissertation: it should be acknowledged that no accomplishment is truly a personal accomplishment and credit should be given to those who deserve it most. Here l'll attempt to summarize a list of those individuals and organizations who have contributed time, energy, encouragement, mentoring, and support. The true list is considerably larger.

First and foremost, I want to thank my adviser Alan Bristow for his encouragement and mentoring. I particularly appreciate the confidence Alan projected in my abilities as a scientist, which translated into the confidence to learn many useful and marketable skills/methods. I should also acknowledge Feruz Ganikhanov and George Spirou for the skills/methods I was able to learn and improve while assisting in the development of a nonlinear-optical microscopy lab. Thank you also to my committee members: David Lederman, Edward Flagg, Paul Cassak, and Nianqiang $\mathrm{Wu}$ for their time and constructive criticism during my oral qualifying exam, oral defense, and concerning this dissertation.

$\mathrm{ZnGeP}_{2}$ samples were graciously provided by Peter Shunemann and Kevin Zawilski at BAE Systems. Without their contribution this work would not have been undertaken. The work was partly sponsored by the West Virginia Higher Education Policy Commission (HEPC.dsr.12.29) and the National Science Foundation (CBET-1233795). Thank you also to the WVNano Initiative for support.

What ultimately made my time at WVU gratifying were the friendships gained and enjoyed, particularly the classmates starting with me in 2007 who provided useful discussion on course material and other less academic topics: Trent Johnson, Kineshma Munbodh, Phil Tabor, William Booth, Jerry Carr, and Adam Brant. I should also thank my friends and labmates: Scott Cushing, Brian Wilmer, Tess Senty, and Derek Bas for useful discussions and many laughs. Lingqing Zhang and Larry Halliburton were kind enough to provide a listening ear and useful advice more often than one might naturally be inclined.

While I won't name them all, I should acknowledge the professors in physics and other subjects who went beyond the call-of-duty to provide a challenging educational experience. I likely wouldn't have taken an interest in physics had it not been for a conceptual physics course at Utah Valley University taught by Phil Matheson who encouraged many of us to consider physics as a degree option. I should also thank Brent Bargeron, who acted as chair of UVU physics, for his friendship and for always showing faith in my abilities.

Finally I would like to thank my parents for teaching me a strong work ethic. Without such a disposition I'm certain I would not have made it through my undergraduate or graduate studies in physics.

-Joseph D. Rowley 


\section{Table of Contents}

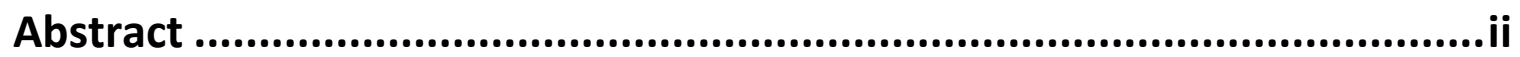

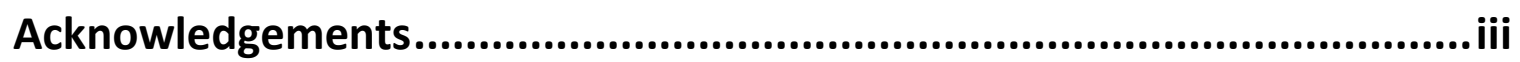

Table of Contents ............................................................................ iv

Chapter 1 Introduction \& Motivation ....................................................1

1.1 Terahertz Pulses: General Properties \& Applications ........................................... 1

1.2 Semiconductors as Broadband Terahertz Sources ............................................. 2

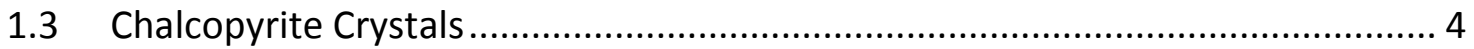

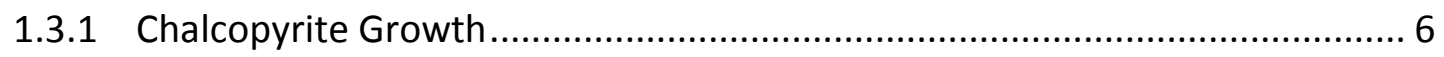

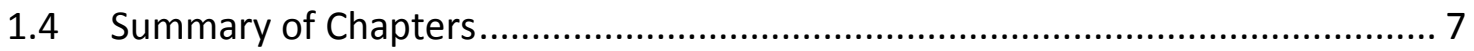

Chapter 2 Terahertz Emission \& Detection ............................................9

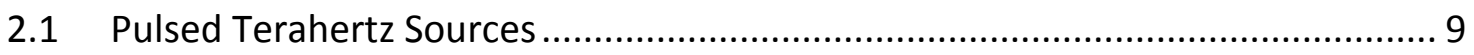

2.1.1 Parametric Generation \& THz Optical Rectification ...................................... 11

2.1.1.1 Terahertz Optical Rectification from Nonlinear Crystals ........................ 17

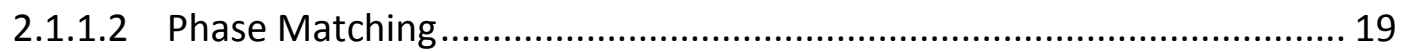

2.1.1.3 Multiphoton Absorption \& Free-Carrier Absorption .............................. 22

2.2 Terahertz Detection ................................................................................... 24

2.2.1 Electro-Optic Sampling ........................................................................... 26

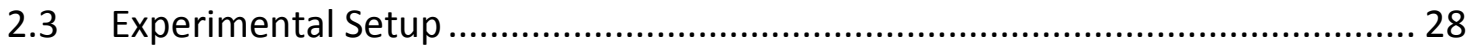

2.3.1 Intensity Autocorrelator ............................................................................ 33

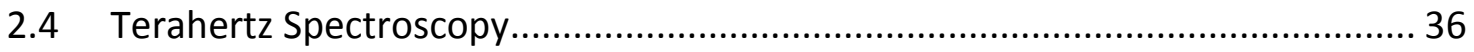

2.4.1 Terahertz Time-Domain Spectroscopy …………........................................ 37

2.4.2 Time-Resolved Terahertz Spectroscopy …….............................................. 40

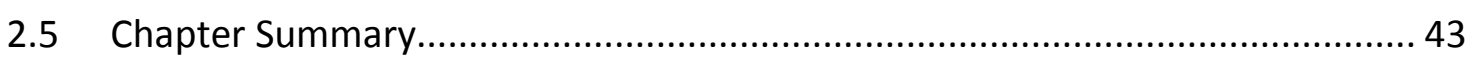

Chapter 3 Optical Rectification in Uniaxial Birefingent Crystals ............45

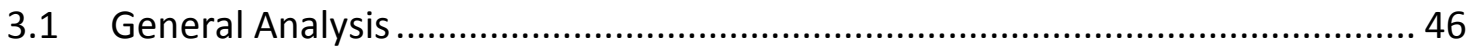

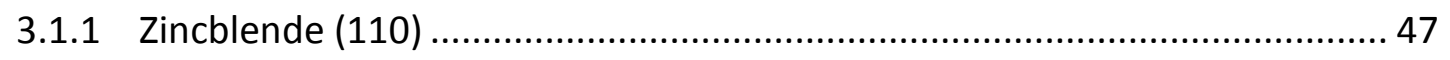




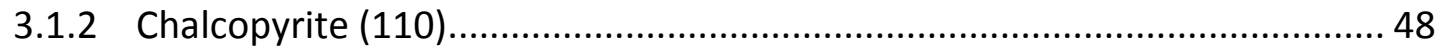

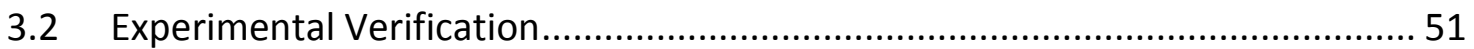

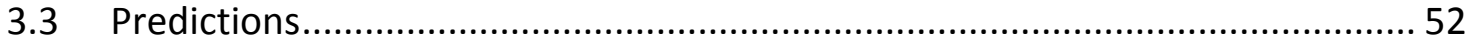

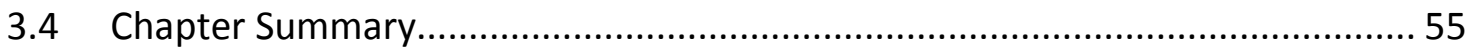

Chapter 4 Phase Matching in Chalcopyrite Structured Crystals.............57

4.1 Chalcopyrite Coherence Length Mapping......................................................... 57

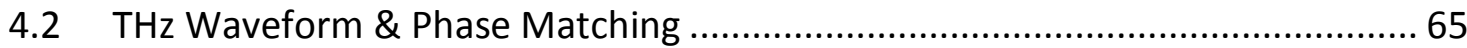

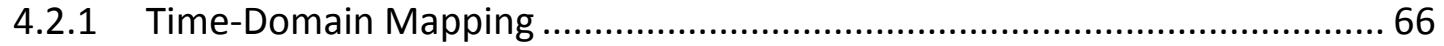

4.2.2 Frequency Domain Mapping \& Modeling ………........................................ 68

4.3 Chapter Summary ..................................................................................... 72

Chapter 5 Optical Rectification in $\mathrm{ZnGeP}_{2}$ : Length \& Intensity Scaling ...74

5.1 Terahertz Waveforms: Low \& High Pump Intensity........................................... 75

5.2 Peak THz Field Amplitudes \& Analysis ............................................................. 77

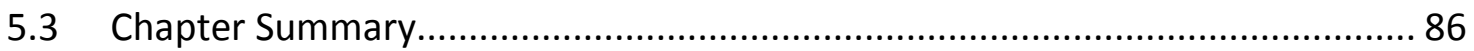

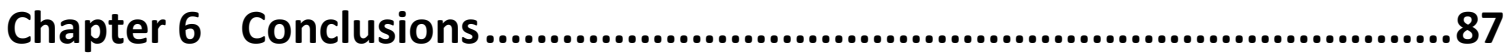

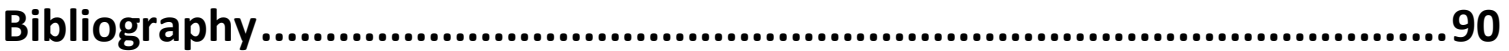




\section{Chapter 1 Introduction \& Motivation}

\subsection{Terahertz Pulses: General Properties \& Applications}

Terahertz $(\mathrm{THz})$ radiation falls between and overlaps with the microwave and farinfrared regions of the electromagnetic spectrum, with the majority of notable and recent developments occurring in the 0.1-10 $\mathrm{THz}$ range. The $\mathrm{THz}$ region is of particular interest for applications due to unique interactions with material systems in this range. For example, many materials opaque to visible radiation are transparent at $\mathrm{THz}$ frequencies and $\mathrm{THz}$ radiation is sensitive to material resonances not accessible at optical, infrared or microwave frequencies. THz spectroscopy has therefore emerged as a powerful spectroscopic tool for determination of static material dielectric properties and for time-resolved measurements of photoconductivity and carrier dynamics [3-6]. THz spectroscopy will be further discussed in Chapter 2.

Exploration and development associated with $\mathrm{THz}$ radiation has lagged in comparison to its infrared and microwave neighbors due to challenges associated with development of sources and detectors. This developmental "THz gap" has, however, diminished in recent decades as new technologies, applications, and fundamental knowledge have emerged at a rapid pace. These applications have filled niches in diverse areas ranging from fundamental science [5,7], medical imaging [8], and communications [9] to quality control in manufacturing, food processing, and information technology, to name a few $[10,11]$. Across these applications the optimal desired time- and frequency-domain attributes of $\mathrm{THz}$ radiation can vary significantly. For example, increasing the current high limit of available electric field amplitudes is of interest for exploration of $\mathrm{THz}$ nonlinear light-matter interactions $[12,13]$. However a combination of broad bandwidth and acceptably high spectral amplitudes (signal-to-noise reduction) are of primary interest for methods such as $\mathrm{THz}$ time-domain spectroscopy (THz TDS) [4] and time-resolved THz spectroscopy (TRTS) [6].

Although many of the promising attributes of $\mathrm{THz}$ radiation were realized in the $1960 \mathrm{~s}$ and earlier [14], the recent $\mathrm{THz}$ revolution, providing the foundation for development of the above techniques and technologies, arguably began in the seventies with the work of Lee et 
al. $[15,16]$ and the successful extension of photoconductive (or Auston) switching to the $\mathrm{THz}$ region in the early 80 s [17-19]. Late in the same decade a pivotal demonstration of free $\mathrm{THz}$ pulse propagation from source to detector opened the way for spectroscopic measurements [20,21]. Pioneering works in THz spectroscopy by Grischkowsky et al. [22], Nuss et al. [23], Arjavalingam et al. [24] and others followed. At about the same time optical rectification emerged as a promising generation scheme [25-27] and has been widely adopted as a table-top method of generating single-cycle THz pulses for THz TDS and TRTS. Other THz sources, derived from femtosecond (fs) pulses, include difference-frequency generation [28], photoconductive switching [29], and four-wave mixing in optically generated plasmas [30]. Listed in order of increasing achievable bandwidth, difference-frequency generation is applied for tunable narrow-band emission whereas optical rectification, photoconductive switching, and four-wave mixing all show broad-bandwidth generation. These methods are further discussed in Chapter 2.

\subsection{Semiconductors as Broadband Terahertz Sources}

Among optical methods, optical rectification of femtosecond laser pulses is an efficient source of broadband $\mathrm{THz}$ pulses. In this method $\mathrm{THz}$ emission results from a time-varying, second-order, nonlinear-optical polarization produced by impulsive difference-frequency mixing of spectral components within a single pump pulse; see Section 2.1.1. The highest conversion efficiencies have been achieved by optical rectification in $\mathrm{LiNbO}_{3}$ at an $0.8 \mu \mathrm{m}$ pump wavelength and using a tilted pulse front to achieve phase matching between the group velocity of the pump pulse and THz spectral-phase velocities [31]; however, in this approach a tradeoff between bandwidth and efficiency is at play: efficiency is diminished significantly for phase matching beyond $\sim 2.5 \mathrm{THz}$. Also, the large pulse front tilt can introduce aberration and reduces optimal efficiency. While achievable $\mathrm{THz}$ pulse energies are significantly greater in $\mathrm{LiNbO}_{3}$ [32], recently published calculations [31,33] suggest that GaP and GaAs should provide a viable alternative to $\mathrm{LiNbO}_{3}$ in the tilted-pulse-front geometry, phase matching above $2.5 \mathrm{THz}$, and pump-photon energies below the onset of two-photon absorption (2PA), which results in a 
rollover of the emitted $\mathrm{THz}$ field amplitude for sufficiently high pump intensities; see Section 2.1.1.3. The effects of $2 \mathrm{PA}$ and three-photon absorption (3PA), at high intensity, can also be mitigated through wide-area pumping, which has been demonstrated in ZnTe [34,35], a semiconductor collinearly phase matched at $\sim 0.8 \mu \mathrm{m}$.

$\mathrm{GaP}$ and GaAs are promising materials for broadband collinear optical rectification at infrared wavelengths longer than, or at the edge of, the tuning range of a Ti:sapphire laser ( 0.7-1.0 $\mu \mathrm{m})$, often emitted by compact fiber laser sources [36-39]. These zincblende semiconductors exhibit pump 2PA below $1.05 \mu \mathrm{m}$ and $1.75 \mu \mathrm{m}$ and optimum collinear phase matching (at $1 \mathrm{THz}$ ) at $1.0 \mu \mathrm{m}$ and $1.4 \mu \mathrm{m}$ respectively. Phase matching is achieved in GaP for moderately thick crystals at pump wavelengths greater than $1.05 \mu \mathrm{m}$, whereas single-crystal GaAs must be significantly limited in thickness to achieve broadband phase matching above $1.75 \mu \mathrm{m}$. Sources emitting near $1.75 \mu \mathrm{m}$ include fiber lasers and optical parametric oscillators.

Periodic variation of the second-order susceptibility tensor has been explored to achieve quasi-phase matching (QPM) in these optically isotropic semiconductors, allowing the use of thicker crystals for which phase matching is not naturally satisfied. QPM was achieved in GaP for $\mathrm{THz}$ emission with $1.55 \mu \mathrm{m}$ pumping using periodically inverted and contacted wafers [40] and electro-optic sampling of $\mathrm{THz}$ pulses with a $0.81 \mu \mathrm{m}$ probe pulse [41]. QPM is achieved in GaAs using diffusion-bound GaAs wafers, orientation-patterned GaAs, and optically-contacted GaAs wafers [42-44]. Due to the above characteristics, GaP and GaAs crystals show particular promise as $\mathrm{THz}$ sources when pumped with compact and low-cost fiber lasers, as has been demonstrated for GaP at $1.06 \mu \mathrm{m}$ pumping and GaAs at $2.0 \mu \mathrm{m}$ and $1.56 \mu \mathrm{m}[37-39,45]$. Compact and cost-effective $\mathrm{THz}$ sources are of clear importance for proliferation of $\mathrm{THz}$ technologies.

$\mathrm{ZnGeP}_{2}$, the focus of the bulk of work in this dissertation, shares attractive qualities for generation by $\mathrm{THz}$ optical rectification with III-V semiconductors, including significantly lower absorption below $\sim 3 \mathrm{THz}$ compared with $\mathrm{ZnTe}$ and $\mathrm{LiNbO}_{3}$, small mismatch between opticalgroup and THz-phase velocities (common in semiconductors), large nonlinear coefficients [36], and routine large-aperture growth [1,2]. Periodic poling of $\mathrm{ZnGeP}_{2}$ has not yet been explored in general. The onset of $2 \mathrm{PA}$ in $\mathrm{ZnGeP}_{2}$ occurs near $1.1 \mu \mathrm{m}$. As will be shown in later chapters, 
$\mathrm{ZnGeP}_{2}$ shows optimized phase matching (at $1 \mathrm{THz}$ ) near $1.2 \mu \mathrm{m}$. Thus, 2PA can be avoided in $\mathrm{ZnGeP}_{2}$ at phase-matched pump frequencies in thick crystals, as in GaP. On the other hand, $\mathrm{ZnGeP}_{2}$ has a nonlinear coefficient about twice that of $\mathrm{GaP}$ and a nonlinear refractive index about 4 times smaller than that of GaAs; see [36]. These attributes give $\mathrm{ZnGeP}_{2}$ a significant advantage over GaP in generation efficiency and GaAs for high intensity pumping and long crystals, where self-focusing and self-phase modulation (SPM) become significant. The birefringence of $\mathrm{ZnGeP}_{2}$ also allows some natural tunability of phase-matched pump/THz frequencies, as will be discussed and demonstrated experimentally. In the chapters that follow $\mathrm{ZnGeP}_{2}$, which was previously unstudied as a broadband $\mathrm{THz}$ source, is demonstrated as a viable alternative to $\mathrm{GaP}$ and GaAs for generation in the near-infrared [46]. Among other chalcopyrite semiconductors considered, $\mathrm{CdGeP}_{2}$ also shows promise as a $\mathrm{THz}$ source due to its potential for broadband phase matching and an optical-to- $\mathrm{THz}$ nonlinear coefficient about twice that of $\mathrm{ZnGeP}_{2}$; see Chapter 4.

\subsection{Chalcopyrite Crystals}

The chalcopyrite crystal structure (named after 'fool's gold' $\mathrm{CuFeS}_{2}$ ) with chemical formula $\mathrm{ABC}_{2}$, common for $\mathrm{I}-\mathrm{III}-\mathrm{VI}_{2}$ and II-IV-V $\mathrm{V}_{2}$ compounds, is tetragonal with $\overline{4} 2 \mathrm{~m}$ point

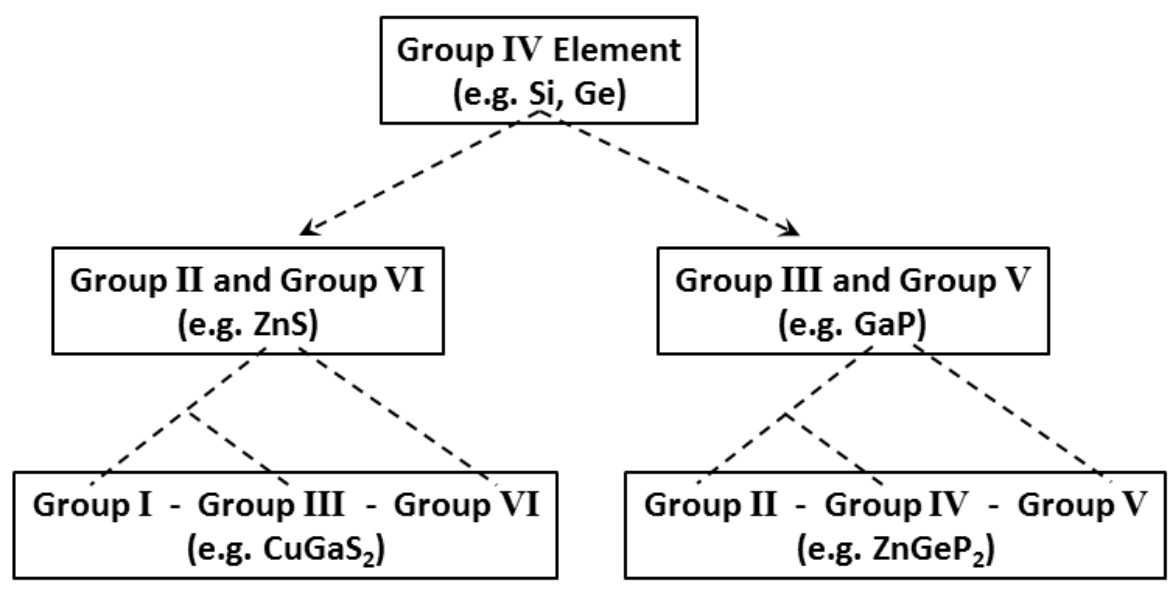

Figure 1.1: Example derivation of tetrahedrally bonded zincblende and chalcopyrite crystal structures from the elemental (diamond) structure by maintaining an electron-to-atom ratio of 4. 
group symmetry. The I-III- $\mathrm{VI}_{2}$ and II-IV-V $\mathrm{V}_{2}$ compounds are ternary analogs of the II-VI and III-V binary semiconductors respectively. Derivation of these structures from elemental tetrahedrally bonded group IV crystals is shown in Figure 1.1 [47]. In contrast to the binary zincblende structure, crystals of chalcopyrite structure are birefringent due to noncubic structure. Because these semiconductors exhibit relatively large second-order nonlinear optical coefficients, due to primarily covalent bonding, they tend to show good efficiency in nonlinearoptical frequency conversion.

As the simplest noncubic ternary analog of the extensively studied zincblende structure, chalcopyrite compounds have been examined out of purely scientific interest but have also found a number of useful applications in solar-cell technology [48], near-infrared generation [49], and show potential for high magnetic-impurity doping [50]. Due to their magnetic properties chalcopyrite semiconductors are of interest for spintronic devices [51,52]. Also, many chalcopyrite semiconductors are predicted to show the topological insulating phase in their native state [53]. Due to these properties and applications, an increase in interest, growth refinement, and availability is probable.

Figure 1.2 shows the conventional unit cells of the elemental diamond structure and its

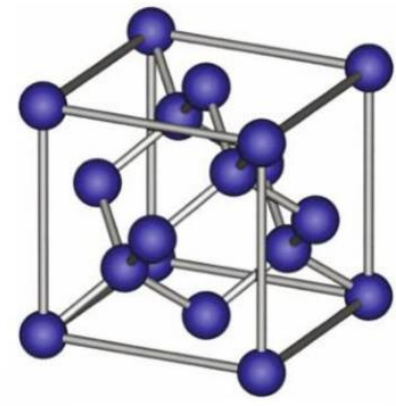

(a)

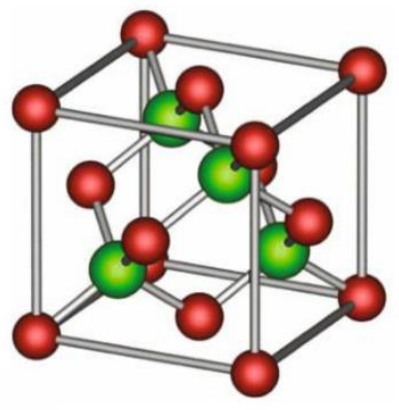

(b)

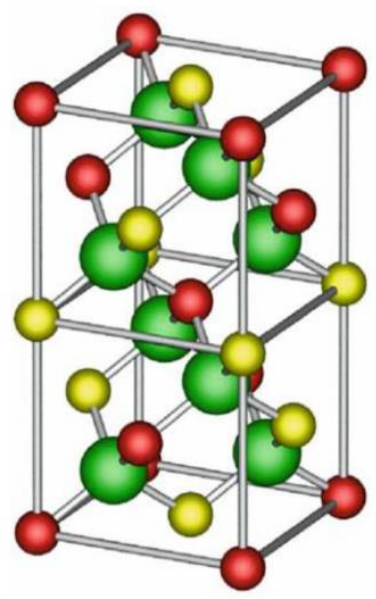

(c)

Figure 1.2: (a) Diamond, (b) zincblende (binary), and (c) chalcopyrite (ternary) crystal structures. In (b) the red spheres represent metallic atoms and the larger green spheres are nonmetal. In (c) red and yellow spheres represent metallic atoms and the larger green spheres are nonmetal. The chalcopyrite conventional unit cell (c) is approximately twice that of the diamond (a) and zincblende (b) structures. 
binary (zincblende) and ternary (chalcopyrite) analogues [54]. The basis of the diamond and zincblende crystal structures consist of atoms at $(0,0,0)$ and $(1 / 4,1 / 4,1 / 4)$ a. For the zincblende structure the atom at $(0,0,0)$ (red) is metallic and the atom at $(1 / 4,1 / 4,1 / 4)$ a (green) is nonmetallic; the metallic and nonmetallic atoms form two FCC sublattices. For the Chalcopyrite structure a nonmetallic anion (green) is bonded to two distinct metallic cations (red and yellow) with four total bonds. Each metallic cation is tetrahedrally coordinated to four nonmetallic atoms with primarily covalent $\mathrm{sp}^{3}$ bonding. For chalcopyrites, the tetragonal aspect ratio $\eta=c /(2 a)$ is in general not equal to 1 due to ordering. Also, because the bond lengths of the two distinct anion-cation bonds are generally unequal the structure is strained, such that the nonmetallic anion is slightly offset from the $(1 / 4,1 / 4,1 / 4)$ a position.

Of the chalcopyrite semiconductors, only a few are commercially available due to their applications in mid-infrared frequency conversion. These include $\mathrm{ZnGeP}_{2}, \mathrm{AgGaS}_{2}$ and $\mathrm{AgGaSe}_{2}$. Quality growth of $\mathrm{CdGeP}_{2}$ has progressed with that of $\mathrm{ZnGeP}_{2}$, but this material is not commercially available; its birefringence is too small for phase-matched frequency conversation in the mid-infrared.

ZnGeP 2 (lattice constants: $a=5.466 \AA$ and $c=10.722 \AA[55]$ ) is a positive uniaxial birefrinegent crystal with an effective direct band-gap of $2.34 \mathrm{eV}$ [56], a large effective nonlinear coefficient, and a high thermal conductivity. As a result of these characteristics, and extensive work in recent decades significantly reducing the infrared absorption of $\mathrm{ZnGeP}_{2}$ (transparency range: $0.74-12 \mu \mathrm{m}$ ), great improvement in mid-infrared frequency conversion has been achieved [57-61]. $\mathrm{ZnGeP}_{2}$ has been applied for narrowband conversion to the $\mathrm{THz}$ frequency range by difference-frequency generation $[62,63]$.

Early growth methods plus optical and electronic properties of chalcopyrite crystals are thoroughly reviewed in a book by Shay and Wernick [47]. A search of the literature suggests that more recent reviews have not been published.

\subsubsection{Chalcopyrite Growth}

Many factors contribute to a choice of crystal-growth method for a given material, these include the compound chemical reactivity and that of its elements, the elemental vapor 
pressures, growth-temperature dissociation pressure of the compound, mealting point of the compound, whether the compound melts or freezes congruently or incongruently, the degree to which a single phase is maintained given deviation from stoichiometry, and the presence/absence and nature of phase transitions [47]. As a result of these many considerations, the difficulty of growth tends to increase significantly for greater numbers of constituent atomic elements; thus, quality growth of ternary semiconductors has lagged behind that of their binary counterparts. Chalcopyrites are generally grown in closed system do to the affinity to oxygen of the constituent elements. Growth methods for I-III-VI $I_{2}$ and II-IV-V include chemical vapor deposition and direct melt techniques including directional solidification. $\mathrm{ZnGeP}_{2}$ specifically has been grown through the latter class of methods: the vertical Bridgman method [1,64,65], vertical gradient freeze [66], and horizontal gradient freeze [2] techniques.

Samples used in the experiments of later chapters were provided by Peter Schunemann and Kevin Zawilski at BAE Systems. The samples were grown with a two-step horizontal gradient freeze seeded-growth technique resulting in large, single-crystal, and crack-free boules [2]. A transparent furnace allows monitoring and manipulation of the growth process. Samples cut in the (110) and (012) planes and three thicknesses $(0.3 \mathrm{~mm}, 0.9 \mathrm{~mm}$, and $3 \mathrm{~mm})$ were obtained with cut planes verified by Laue crystallography. Measurements of the nearinfrared and $\mathrm{THz}$ linear absorption coefficients corroborate the high quality of the samples. Uniformity of absorption and birefringence in the provided samples was verified by our collaborators using a polarimeter at $2 \mu \mathrm{m}$ [67].

\subsection{Summary of Chapters}

Characterization and analysis of $\mathrm{THz}$ generation by optical rectification in $\mathrm{ZnGeP}_{2}$ and other chalcopyrites will occupy the majority of this dissertation. In Chapter 2 the particulars of pulsed $\mathrm{THz}$ generation and detection are addressed and the $\mathrm{THz}$ spectroscopy setup, constructed and utilized for collection and detection of emitted THz transients, is presented. Both optical rectification (generation) and electro-optic sampling (detection) are discussed in this context. Preliminary demonstrations of $\mathrm{THz}$ time-domain spectroscopy and time-resolved $\mathrm{THz}$ spectroscopy are also presented. 
In Chapter 3, collinear optical rectification in uniaxial birefringent crystals is explored experimentally using $\mathrm{ZnGeP}_{2}$ as a model system. Data describing generation efficiency as a function of crystal/pump orientation are modeled and discussed including predictions of the most efficient planes of generation in chalcopyrite semiconductors.

Chapter 4 deals exclusively with phase matching. The phase matching characteristics of several chalcopyrite crystals, for which experimental THz and near-infrared dispersion relations are available, are determined by mapping the coherence length: a characteristic length over which efficient generation is achieved for a given pump wavelength and THz frequency. The potential of these chalcopyrite semiconductors is discussed based on the calculated coherence length and relevant tabulated parameters. Also in Chapter 4, phase-matched pump-photon energies are experimentally verified for two efficient orientations of $\mathrm{ZnGeP}_{2}$ by observing the peak-to-peak field amplitude of $\mathrm{THz}$ generation as a function of tuned pump wavelength. The effects of phase matching beyond peak-to-peak efficiency are explored by mapping the field amplitude of $\mathrm{THz}$ transients as a function of pump photon energy and time. Fourier transformation of each transient is found to fit well with an applied model.

Finally, in Chapter 5 the scalability of collinear optical rectification in $\mathrm{ZnGeP}_{2}$ as a function of pump intensity and crystal length is explored. Through analysis of the emitted $\mathrm{THz}$ waveform, factors limiting intensity and length scalability are identified including 3PA resulting in enhanced free-carrier absorption and SPM. 


\section{Chapter 2 Terahertz Emission \& Detection}

Electromagnetic sources in the $\mathrm{THz}$ range include monochromatic sources such as backward wave oscillators [68] and quantum cascade lasers [69], large scale facility sources such as free electron lasers [70] and synchrotrons [71], and table top sources utilizing pulsed lasers. The latter class of pulsed sources is largely responsible for the proliferation of $\mathrm{THz}$ technologies within the last few decades and is the focus of this chapter and dissertation. In addition, methods of detecting pulsed $\mathrm{THz}$ radiation are discussed with emphasis on electrooptic sampling. These sources and detectors are discussed in the context of their application in THz spectroscopy systems. The THz spectroscopy setup applied in the experiments described in later chapters is summarized as well as the autocorrelation system constructed and automated for determining near-infrared pulse durations.

\subsection{Pulsed Terahertz Sources}

A number of pulsed tabletop $\mathrm{THz}$ sources are now available for $\mathrm{THz}$ spectroscopy including optical rectification in collinear or tilted-pulse-front geometry, use of photoconductive switches, difference-frequency mixing of two tunable sources, four-wavemixing in a plasma, and plasma generation by a single pump beam [72]. The physical mechanisms resulting in $\mathrm{THz}$ emission and practical considerations in choosing between these methods are summarized here.

Photoconductive switching is unique among pulsed $\mathrm{THz}$ generation methods because a high voltage source provides energy for $\mathrm{THz}$ emission in addition to the pump pulse. In this method the pump pulse, with above band-gap photon energy impinges on a semiconductor optically generating electron-hole pairs; these pairs are subsequently accelerated by a strong external electric field, resulting in transient emission of $\mathrm{THz}$ electromagnetic radiation [73]. Difference-frequency generation is a parametric second-order nonlinear effect whereby emission is achieved at the difference frequency of two pump pulses which simultaneously and parametrically interact with material bound electrons [74]. Similarly, optical rectification is 
nearly degenerate difference-frequency generation (see Section 2.1.1); that is, optical rectification is difference-frequency generation between modes within the bandwidth of a single pump pulse. Four-wave mixing in plasma may be described as a higher-order nonlinear interaction [30], typically between two harmonically related fundamental and second-harmonic pulses. The plasma is generated in air or other gas by the pulses themselves and THz emission is governed by the transient motion of these charges. Alternatively, THz emission from plasma, generated by a single frequency pump, results from ionization and acceleration of electrons by a driving ponderomotive force, resulting from the intense electric fields at the focus of the pulse [30].

The generation method of choice, from the above, will depend on the $\mathrm{THz}$ frequency range of interest, the necessity of high peak field amplitudes, as well as the frequency, tunability, and peak intensity of available pump pulses; for example, $\mathrm{THz}$ generation from plasmas is only possible using laser amplifiers with high peak-pulse intensities. Plasma generation is less efficient than generation in crystals, but scalability of efficiency with pump intensity is not significantly limited by material properties such as dispersion, phase matching, intensity damage thresholds, multi-photon absorption, free-carrier absorption, or self-phase modulation. Because difference-frequency generation in a nonlinear crystal is a source of narrow-band $\mathrm{THz}$ emission, continuous tuning of the phase-matching condition is generally necessary for spectroscopic applications. This requires birefringence of the $\mathrm{THz}$ emitter or tuning of one or both of the pump frequencies. Broadband $\mathrm{THz}$ generation by photoconductive switches shows good potential for generation of high-energy $\mathrm{THz}$ pulses, but exhibits limited generation bandwidth compared with optical rectification, depending on the crystal and pump wavelength of choice. Also, the amplitude of emission saturates at relatively low pump intensities and the GaAs surface may degrade due to high-voltage arcing across electrodes [34].

Optical rectification was chosen as the source for a $\mathrm{THz}$ spectroscopy setup discussed in this dissertation due to good efficiency, potential for broadband emission, mechanical compactness, and ease of implementation. Optical rectification is well established and extensively applied as a source in standard THz spectroscopy setups [73]. 


\subsubsection{Parametric Generation \& THz Optical Rectification}

In general, electromagnetic radiation, which approximates a plane wave, fulfills the Maxwell wave equation,

$$
\left(\nabla^{2}-\frac{1}{c^{2}} \frac{\partial^{2}}{\partial t^{2}}\right) \mathbf{E}=\mu_{o}\left(\frac{\partial \mathbf{J}}{\partial t}+\frac{\partial^{2} \mathbf{P}}{\partial t^{2}}\right)
$$

where $\mathbf{E}$ is the electric field, $\mathbf{J}$ represents a charge current density, $\mathbf{P}$ is the material bound charge polarization, $C$ is the speed of light, and $\mu_{o}$ is the permeability of free space. This equation indicates that dynamic charge currents and dynamic material polarizations act as sources of electromagnetic radiation; that is, the source term containing $\mathbf{J}$ will describe radiation emitted from $\mathrm{THz}$ sources such a photoconductive switches and air-plasma generation, which rely on accelerated ions. The source term containing $\mathbf{P}$ will describe $\mathrm{THz}$ emission by difference-frequency generation and $\mathrm{THz}$ optical rectification, resulting from dynamics of bound electrons. Because optical rectification is the method of generation examined in the experiments of later chapters, the source term containing $\mathbf{J}$ will be neglected and discussion will focus on the bound charge polarization. Specifically, the polarization resulting from nonlinear-optical, parametric light-matter interactions will be discussed.

Modeling one physical quantity as a linear function of another is frequently a simplifying approximation. Such is the case for the fundamental assumption of linear optics; that is, each dielectric bound charges resides in a perfectly parabolic potential and experiences a linear restoring force in response to applied fields. More specifically, a linear relationship between polarization and applied electric field is assumed: $P(t)=\varepsilon_{o} \chi E(t)$. For sufficiently high electricfield amplitudes this assumption is violated: each bound charge will be sensitive to the typically nonparabolic potential in which it resides and the polarization will display a nonlinear response to applied dynamic or static electric fields. The problem of defining the polarization response to an applied field is then that of an anharmonic oscillator and an approximating series expansion greatly simplifies the problem [74]. The linear and nonlinear dependence of the polarization resulting from an electric field is then phenomenologically represented as a power series [75]: 


$$
\begin{aligned}
\mathbf{P}(\mathbf{r}, t)= & \varepsilon_{o} \int_{-\infty}^{\infty} \boldsymbol{\chi}^{(1)}\left(\mathbf{r}-\mathbf{r}^{\prime}, t-t^{\prime}\right) \cdot \mathbf{E}\left(\mathbf{r}, t^{\prime}\right) d \mathbf{r}^{\prime} d t \\
+ & \varepsilon_{o} \int_{-\infty}^{\infty} \boldsymbol{\chi}^{(2)}\left(\mathbf{r}-\mathbf{r}_{1}, t-t_{1} ; \mathbf{r}-\mathbf{r}_{2}, t-t_{2}\right): \mathbf{E}\left(\mathbf{r}_{1}, t_{1}\right) \times \mathbf{E}\left(\mathbf{r}_{2}, t_{2}\right) d \mathbf{r}_{1} d t_{1} d \mathbf{r}_{2} d t_{2} \\
& +\varepsilon_{o} \int_{-\infty}^{\infty} \boldsymbol{\chi}^{(3)}\left(\mathbf{r}-\mathbf{r}_{1}, t-t_{1} ; \mathbf{r}-\mathbf{r}_{2}, t-t_{2} ; \mathbf{r}-\mathbf{r}_{3}, t-t_{3}\right): \\
& \mathbf{E}\left(\mathbf{r}_{1}, t_{1}\right) \times \mathbf{E}\left(\mathbf{r}_{2}, t_{2}\right) \mathbf{E}\left(\mathbf{r}_{3}, t_{3}\right) d \mathbf{r}_{1} d t_{1} d \mathbf{r}_{2} d t_{2} d \mathbf{r}_{3} d t_{3}+\ldots
\end{aligned}
$$

The first term in Equation (2.2) represents the linear optical response and the following two terms represent the second-order and third-order nonlinear polarization responses. $\varepsilon_{o}$ is the electric permittivity of free space and $\chi^{(n)}$ is the $\mathrm{nth}$ order susceptibility tensor of rank $\mathrm{n}+1$. The time dependence of the susceptibilities accounts for a non-instantaneous material response to the applied field(s); a time dependent susceptibility is alternatively referred to as a response function. All materials will exhibit a third order nonlinear response at sufficiently high field amplitudes, but the second-order response vanishes for materials with inversion symmetry. For such materials the polarization must change sign with the sign of the electric field; it is therefore not physical to include polarization terms with even powers of the field. Optical rectification, a second-order nonlinear effect, only occurs in materials which are noncentrosymmetric. An instantaneous polarization response may be assumed in Equation (2.2) for nonresonant parametric interactions and material response times shorter than the excitation- (pump-) pulse duration.

An optical parametric process is a light-matter, photon scattering, interaction in which the quantum state of a bound electron is unchanged. The virtual energy levels accessed to facilitate such a single or multiphoton interaction can only exist on a short time scale, on the order of $\hbar / \Delta E$, restricted by the Heisenberg time-energy uncertainty relation; $\Delta E$ is the energy difference between a virtual energy level and the nearest real level. For photon energies in the optical and near-infrared range the interaction time $\left(\sim 10^{-15} \mathrm{~s}\right)$, during which multiple photons may scatter, is small in comparison to typical $100 \mathrm{fs}$ pulse durations, justifying the assumption of an instantaneous polarization response. The probability of multiphoton scattering must be high for a given parametric interaction to be non-negligible. Due to the tiny allowed interaction time, higher multiphoton parametric interactions require high photon densities to be observable; that is, nonlinear interactions are only easily observable for high 
intensity driving electric fields. The above properties, understood from a quantum perspective, are accounted for by the polarization defined by Equation (2.2) with real susceptibilities: the first term representing single photon parametric excitation and emission, the second-order term representing two-photon parametric scattering, and the third-term representing threephoton parametric scattering.

Let $\mathbf{E}$ be represented as a superposition of monochromatic plane waves

$$
\mathbf{E}(\mathbf{r}, t)=\sum_{i} \mathbf{A}_{i}(t) \exp \left(i \mathbf{k}_{i} \cdot \mathbf{r}-i \omega_{i} t\right)+c . c .
$$

with $\mathbf{A}(t)$ representing a complex slowly varying field amplitude; $\mathbf{k}_{i}$ and $\omega_{i}$ are the wavevectors and frequencies of individual modes. Taking the Fourier transform of (2.2) then gives the frequency dependent polarization:

$$
\mathbf{P}(\mathbf{k}, \omega)=\mathbf{P}^{(1)}(\mathbf{k}, \omega)+\mathbf{P}^{(2)}(\mathbf{k}, \omega)+\mathbf{P}^{(3)}(\mathbf{k}, \omega)+\ldots
$$

The first, second, and third order polarizations are [74,75]:

$$
\begin{aligned}
& \mathbf{P}^{(1)}(\mathbf{k}, \omega)=\varepsilon_{o} \boldsymbol{\chi}^{(1)}(\mathbf{k}, \omega) \cdot \mathbf{E}(\mathbf{k}, \omega) \\
& \mathbf{P}^{(2)}(\mathbf{k}, \omega)=D \varepsilon_{o} \boldsymbol{\chi}^{(2)}\left(\mathbf{k}, \omega ; \omega_{1}, \omega_{2}\right): \mathbf{E}\left(\mathbf{k}_{1}, \omega_{1}\right) \mathbf{E}\left(\mathbf{k}_{2}, \omega_{2}\right) \\
& \mathbf{P}^{(3)}(\mathbf{k}, \omega)=D \varepsilon_{o} \boldsymbol{\chi}^{(3)}\left(\mathbf{k}, \omega ; \omega_{1}, \omega_{2}, \omega_{3}\right): \mathbf{E}\left(\mathbf{k}_{1}, \omega_{1}\right) \mathbf{E}\left(\mathbf{k}_{2}, \omega_{2}\right) \mathbf{E}\left(\mathbf{k}_{3}, \omega_{3}\right) ;
\end{aligned}
$$

the nth order susceptibility is:

$$
\begin{aligned}
\boldsymbol{\chi}^{(n)}(\mathbf{k}, \omega) & =\int_{-\infty}^{\infty} \boldsymbol{\chi}^{(n)}\left(\mathbf{r}, t ; t_{1}, t_{2}, \ldots, t_{n}\right) \times \\
& e^{-i\left\{\mathbf{k}_{1}\left(\mathbf{r}-\mathbf{r}_{1}\right)-\omega_{1}\left(t-t_{1}\right)+\ldots+\mathbf{k}_{n}\left(\mathbf{r}-\mathbf{r}_{n}\right)-\omega_{n}\left(t-t_{n}\right)\right\}} d \mathbf{r}_{1} d t_{1} \ldots d \mathbf{r}_{n} d t_{n} .
\end{aligned}
$$

$D$ is the number of distinct permutations of the frequency components. For higher-order nonlinear polarizations, $\mathbf{k}$ and $\omega$ are the wavevector and frequency of generated radiation and are defined by conservation of momentum and energy respectively; that is, the susceptibility tensor depends on the particular nonlinear parametric light matter interaction of interest. The second order nonlinear polarization expressed in component form is:

$$
P_{i}^{(2)}\left(k_{3}, \omega_{3}\right)=\varepsilon_{o} D \sum_{j k} \chi_{i j k}^{(2)}\left(k_{3}, \omega_{3} ; \omega_{1}, \omega_{2}\right) E_{j}\left(k_{1}, \omega_{1}\right) E_{k}\left(k_{2}, \omega_{2}\right)
$$


$i, j$, and $k$ are dummy indices for the $x, y$, and $z$ directions in the crystallographic frame of reference.

It is constructive to consider an electric field consisting of only two frequency components:

$$
\mathbf{E}(t)=\mathbf{E}_{1}(t)+\mathbf{E}_{2}(t)=\mathbf{A}_{1}(t) \exp \left(i k_{1} z-i \omega_{1} t\right)+c . c .+\mathbf{A}_{2}(t) \exp \left(i k_{2} z-i \omega_{2} t\right)+\text { c.c. }
$$

Ignoring, for the sake of illustration, the vector fields and potential material anisotropy, the second-order nonlinear polarization resulting from these fields is (Equation (2.7)):

$$
\begin{aligned}
P^{(2)}(k, \omega) & =\varepsilon_{o} \chi^{(2)} E_{1}\left(k_{1}, \omega_{1}\right) E_{2}\left(k_{2}, \omega_{2}\right) \\
& =\varepsilon_{o} \chi^{(2)}\left\{A_{1}(t) A_{2}(t) \exp \left[i\left(k_{1}+k_{2}\right) z-i\left(\omega_{1}+\omega_{2}\right) t\right]\right. \\
& \left.+A_{1}(t) A_{2}^{*}(t) \exp \left[i\left(k_{1}-k_{2}\right) z-i\left(\omega_{1}-\omega_{2}\right) t\right]\right\}+ \text { c.C. }
\end{aligned}
$$

Equation (2.9) shows the potential for multiple field components to drive the nonlinear bound charge polarization resulting in polarization dynamics at both the sum- and differencefrequency of extant frequency components. Considering Equation (2.9) under the particular constraint of $\omega_{1}=\omega_{2}$ and $k_{1}=k_{2}$ gives

$$
P^{(2)}(k, \omega)=\varepsilon_{o} \chi^{(2)}\left\{A(t)^{2} \exp (2 i k z-2 i \omega t)+c . c .\right\}+2 \varepsilon_{o} \chi^{(2)} A(t) A^{*}(t) .
$$

The first term of this equation shows degenerate sum-frequency generation, and the second shows degenerate difference-frequency generation, otherwise known as second-harmonic generation and optical rectification respectively. It may be noted that for ideal materials all second-order parametric processes scale quadratically with the applied electric-field amplitude. However, higher-order nonlinear effects such as multiphoton absorption and self-phase modulation may affect the incident field amplitude disrupting this quadratic dependence. These effects will be addressed in later sections and chapters.

Figure 2.1 shows an illustrative toy model for a single-frequency driving field at (a) low and (b) high optical intensity. The nonlinear static polarization response (solid line: $P$ vs. E) to an applied electric field results from the asymmetric potentials of bound ions and deviates significantly from that of a linear response (dotted line). The response thus includes a linear and second-order nonlinear contribution. For low field amplitudes the time-dependent polarization 

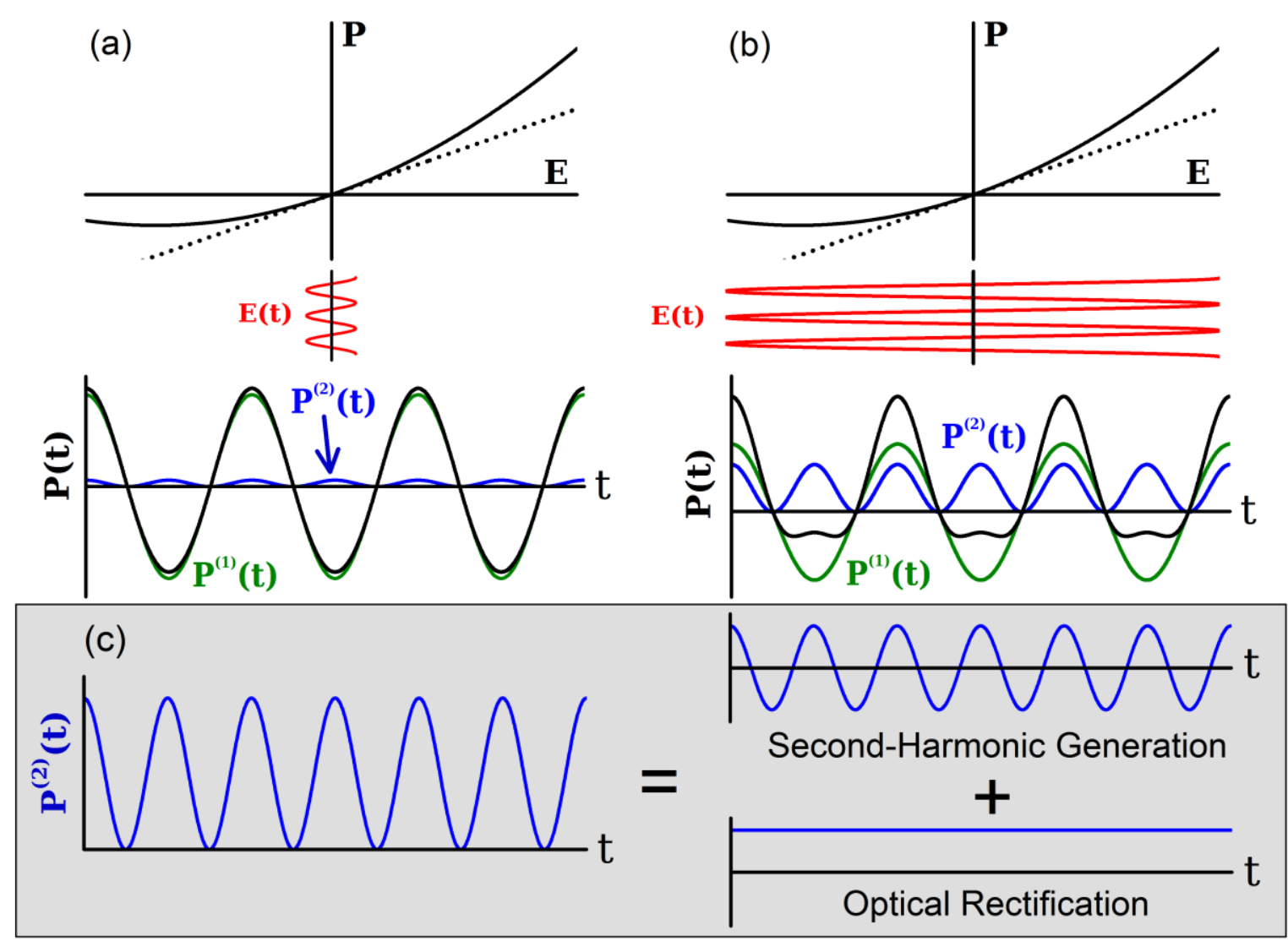

Figure 2.1: (a) and (b) show polarization dependence on field amplitude in a material lacking inversion symmetry. In the lower fields of (a) and (b) the total time dependent polarization is shown in black, the linear contribution to the polarization is green and the second order contribution is blue. For small applied fields (a) the second order response is negligible. For a field amplitude ten times larger (b) the polarization shows a significant nonlinear response. (c) shows Fourier components of the second order polarization: a DC offset (optical rectification) and a component with twice the frequency of the applied field (second harmonic generation).

response $P(t)$ is approximately linear resulting in transmission at the driving frequency. For high field amplitudes $P(t)$ shows a strong nonlinear contribution $P^{(2)}(t)$ (blue). Figure 2.1 (c) shows the second-order polarization oscillating at twice the frequency of the incident field amplitude with a DC offset. These Fourier components of the second order response result in radiative second-harmonic generation and nonradiative optical rectification, otherwise referred to as degenerate sum-frequency and degenerate difference-frequency generation respectively.

As indicated by Equation (2.1) the DC polarization generated by optical rectification with a CW driving field will not radiate. However, if a pulsed field is applied the induced DC polarization evolves on the time scale of the pulse duration, resulting in broadband radiation in 
the $\mathrm{THz}$ frequency range; the bandwidth of emission strongly depends on the pulse duration; however, this conceptual picture is not complete. Because the femtosecond pulses are not monochromatic, THz optical rectification is most accurately represented as nearly degenerate difference-frequency generation: difference-frequency generation between the spectral components contained within the bandwidth of a single pump pulse. Because standard ultrafast pulses exhibit a continuous range of frequencies, generated $\mathrm{THz}$ pulses also contain a continuous frequency bandwidth determined by the pump pulse spectral properties. From this perspective the nonlinear polarization as a function of beating frequency $\Omega$ is [76]

$$
P_{i}^{(2)}(\Omega)=\sum_{j, k} \varepsilon_{o} \chi_{i j k}^{(2)}\left(\Omega=\omega_{1}-\omega_{2}\right) \int_{-\infty}^{\infty} E_{j}\left(\omega_{1}=\Omega+\omega_{2}\right) E_{k}\left(\omega_{2}\right) d \omega_{2},
$$

and in the time domain

$$
\mathbf{P}^{(2)}(t)=\frac{1}{2 \pi} \int_{-\infty}^{\infty} \mathbf{P}^{(2)}(\Omega) \exp (-i \Omega t) d \Omega
$$

In the dipole approximation the radiated electric field in the far field is [77]

$$
\mathbf{E}_{r}(\Omega)=-k^{2} \hat{n} \times(\hat{n} \times \mathbf{P}(\Omega)) \frac{\exp (i k r)}{r},
$$

such that $E_{r}(\Omega) \propto \Omega^{2} P(\Omega)$, where $P(\Omega)$ is the dipole polarization and $k=\frac{\Omega}{C}$. The field in the time domain according to Equation (2.12) is then:

$$
E_{r}(t) \propto \frac{\partial^{2}}{\partial t^{2}} P(t) \propto \chi^{(2)} \int_{-\infty}^{\infty} \exp (-i \Omega t) \Omega^{2} d \Omega \int_{-\infty}^{\infty} E_{j}\left(\omega_{1}=\Omega+\omega_{2}\right) E_{k}\left(\omega_{2}\right) d \omega_{2} .
$$

This simple result for the generated $\mathrm{THz}$ time-domain waveform neglects a number of important factors. A more descriptive model involves the simultaneous solution of equations (2.1) and (2.11), a nontrivial problem considered by Faure et al. [78]. Their solution accounts for the effects of dispersion and absorption of the $\mathrm{THz}$ field as well as phase mismatch between the laser and $\mathrm{THz}$ pulses. This more complete theoretical approach is referenced in modeling data presented and discussed in Chapter 4. Factors, not yet defined/discussed, affecting the efficiency of $\mathrm{THz}$ optical rectification include the tensor nature of the second-order susceptibility, phase mismatch between the pump and generated $\mathrm{THz}$ pulse, and the 
detrimental effects of multiphoton absorption. These effects are addressed in the following subsections.

\subsubsection{Terahertz Optical Rectification from Nonlinear Crystals}

In real applications, the tensor nature of the second-order susceptibility is of paramount importance: neglecting other effects, the magnitudes and distribution of nonzero tensor elements primarily determine the efficiency of generation given the crystal-frame pumppolarization orientation. The distribution of nonzero elements is determined by the lattice point group symmetry. Here, the orientation dependence of $\mathrm{THz}$ emission is determined for zincblende/chalcopyrite crystals. This calculation identifies the (110) plane as the plane of highest generation efficiency in zincblende semiconductors. An experiment specific calculation of emission efficiency as a function of linear-pump-polarization angular dependence in the zincblende (110) plane will be presented in Section 3.1.1. Effects of birefringence are considered in Section 3.1.2.

The second-order nonlinear susceptibility $\chi_{i j k}^{(2)}$ is conveniently expressed in a contracted notation with the subscripts $\mathrm{j}$ and $\mathrm{k}$ replaced by a single index:

$$
x x=1, y y=2, z z=3, y z=z y=4, x z=z x=5, x y=y x=6,
$$

with $d_{m n}=\chi_{i j k}^{(2)} / 2$. Using this notation the crystal-frame polarization components from Equation (2.7) may be represented in a two-dimensional plan as

$$
\left(\begin{array}{l}
P_{x} \\
P_{y} \\
P_{z}
\end{array}\right)=\varepsilon_{o}\left(\begin{array}{cccccc}
d_{11} & d_{12} & d_{13} & d_{14} & d_{15} & d_{16} \\
d_{21} & d_{22} & d_{23} & d_{24} & d_{25} & d_{26} \\
d_{31} & d_{31} & d_{33} & d_{34} & d_{35} & d_{36}
\end{array}\right)\left(\begin{array}{c}
E_{x}^{2} \\
E_{y}^{2} \\
E_{z}^{2} \\
2 E_{y} E_{z} \\
2 E_{z} E_{x} \\
2 E_{x} E_{y}
\end{array}\right) .
$$

Depending on the degree of crystal symmetry, only a few tensor elements are nonzero. For the zincblende and chalcopyrite crystal structures, with $\overline{4} 3 m$ and $\overline{4} 2 m$ point group symmetries respectively, the nonzero elements are $d_{14}, d_{25}$, and $d_{36}$. For zincblende crystals all these 
elements are equal. For chalcopyrite structured crystals $d_{14}=d_{25}$, but these elements are not necessarily equal to $d_{36}$ [74]. In the following analysis, the general condition $d_{14}=d_{35} \neq d_{36}$ is considered. An arbitrary field orientation in the crystal frame of reference may be expressed as

$$
\vec{E}_{o}=E_{o}\left(\begin{array}{c}
\sin \theta \cos \phi \\
\sin \theta \sin \phi \\
\cos \theta
\end{array}\right) \text {. }
$$

$E_{o}$ is the incident-pump-field amplitude, $\theta$ is the polar angle relative to the z-axis, and $\phi$ is the azimuthal angle defined relative to the x-axis. Using Equation (2.16) and (2.17) the nonlinear polarization is defined as

$$
\begin{aligned}
\left(\begin{array}{l}
P_{x} \\
P_{y} \\
P_{z}
\end{array}\right)=\varepsilon_{o} E_{o}^{2}\left(\begin{array}{cccccc}
0 & 0 & 0 & d_{14} & 0 & 0 \\
0 & 0 & 0 & 0 & d_{14} & 0 \\
0 & 0 & 0 & 0 & 0 & d_{36}
\end{array}\right)\left(\begin{array}{c}
\sin ^{2} \theta \cos ^{2} \phi \\
\sin ^{2} \theta \sin ^{2} \phi \\
\cos ^{2} \theta \\
2 \sin \theta \cos \theta \sin \phi \\
2 \sin \theta \cos \theta \cos \phi \\
2 \sin ^{2} \theta \sin \phi \cos \phi
\end{array}\right) \\
=2 \varepsilon_{o} E_{o}^{2} \sin \theta\left(\begin{array}{c}
d_{14} \cos \theta \sin \phi \\
d_{14} \cos \theta \cos \phi \\
d_{36} \sin \theta \sin \phi \cos \phi
\end{array}\right) .
\end{aligned}
$$

The electric-field amplitude of the emitted $\mathrm{THz}$ radiation is proportional in direction and amplitude to the total nonlinear polarization. Thus, the dependence of $\mathrm{THz}$ emission intensity on crystal orientation relative to a linear pump polarization is

$$
I_{T H z}(\theta, \phi) \propto\left|\vec{P}^{2}\right|=4 \varepsilon_{o}^{2} E_{o}^{4} \sin ^{2} \theta\left(4 d_{14}^{2} \cos ^{2} \theta+d_{36}^{2} \sin ^{2} \theta \sin ^{2}(2 \phi)\right) .
$$

For the chalcopyrite structure, without defining the relative strengths of $d_{14}$ and $d_{36}$, the most efficient plane cannot be absolutely known from this relation. For zincblende crystals, all nonzero tensor elements are equal and the planes of maximum emission are defined by $\phi=\pi / 4,3 \pi / 4$ such that $\sin ^{2}(2 \phi)=1$; that is, the pump polarization will be in the $\{110\}$ 
planes for maximum efficiency. Under this restraint the intensity is maximized for $\boldsymbol{\theta}=54.74^{\circ}$ and $125.26^{\circ}$ between $0^{\circ}$ and $180^{\circ}$. Physically this corresponds to aligning the pump polarization parallel to the longest crystal bond between two zincblende atomic types.

For most semiconductors the second-order susceptibility for near-infrared-to-THz optical rectification is determined from known electro-optic tensor elements. The second-order susceptibility is related to the material electro-optic tensor $r_{i j k}$ by [79]: $\chi_{i j k}=-\left(n^{4} / 2\right) r_{i j k}$ where $n$ is the refractive index of the material. This direct relationship is expected; indeed, optical rectification is commonly referred to as the "inverse electro-optic effect" in the literature: the electro-optic effect is a change in polarizability due to an applied electric field and optical rectification is the generation of a DC electric field due to an induced polarization. The electro-optic effect will be further discussed in Section 2.2 in the context of $\mathrm{THz}$ detection methods.

An analysis similar to the above will give the most efficient orientations for other crystal symmetries exhibiting second-order nonlinearity; however, in later sections it will be demonstrated, experimentally and theoretically, that birefringence is an important consideration when calculating emission efficiency as a function of crystal/pump polarization orientation.

\subsubsection{Phase Matching}

Another factor affecting the efficiency of $\mathrm{THz}$ emission is the degree to which phasematching is satisfied. For two frequencies, $\omega_{1}$ and $\omega_{2}$, within the bandwidth of the pump pulse, the efficiency of generation at a single THz frequency $\Omega=\omega_{1}-\omega_{2}$ is quantified as [80]:

$$
\eta=\frac{P_{T H z}}{P_{\text {Pump }}}=\frac{8 \pi^{2} d_{\text {eff }}^{2} L^{2} I_{\text {Pump }}}{\varepsilon_{o} n_{\text {Pump }}^{2} n_{T H z} c \lambda_{T H z}^{2}} \frac{\sin ^{2}(\Delta k L / 2)}{(\Delta k L / 2)^{2}}
$$

where $I_{\text {Pump }}$ is the pump intensity, $L$ is the crystal length, and $d_{\text {eff }}$ is the effective nonlinearity which depends on crystal orientation relative to a linear pump polarization. Equation (2.19) assumes the interaction of plane waves and an ideal crystal with no absorption at $\mathrm{THz}$ or nearinfrared frequencies. The phase mismatch $(\Delta k)$ is the degree to which the phase-matching 
condition $k_{T H z}=k_{1}-k_{2}$ is broken; that is, $\Delta k=k_{T H z}-\left(k_{1}-k_{2}\right)$. From Equation (2.9) it is clear that $k_{1}-k_{2}=2 \pi\left(n_{1} / \lambda_{1}-n_{2} / \lambda_{2}\right)$ defines the speed of the second-order polarization wave generated by the mixing of fields at $\omega_{1}$ and $\omega_{2}$; the phase-matching condition is perfectly satisfied when the phase velocity and direction of the generated $\mathrm{THz}$ mode, defined by the wavevector $k_{T H z}=2 \pi n_{T H z} / \lambda_{T H z}$, matches the propagation speed and direction of the generating polarization wave. When the generated $\mathrm{THz}$ mode and generating pulse get out of step, interference effects prevent efficient energy transfer from the latter to the former in crystals of significant length. As will be discussed, this length is typically quantified by definition of the coherence length.

Because $\lambda_{T H z} \gg \lambda_{o p t, N I R}$ and $\lambda_{T H z}^{2}$ is found in the denominator, Equation (2.20) shows that the efficiency of optical rectification $\left(\sim 10^{-6}\right)$ is significantly smaller than that for narrowband difference-frequency generation within the optical or near-infrared ranges. Also, $P_{\mathrm{THz}} \propto d_{\text {eff }}^{2} L^{2} I_{\text {Pump }}^{2}$ or $E_{\mathrm{THz}} \propto d_{\text {eff }} L I_{\text {Pump }}$, so ideally the generated electric-field amplitude scales linearly with crystal length and pump intensity. The term accounting for phase mismatch $(\Delta k)$ demonstrates a tradeoff between generation efficiency and generation bandwidth of phase-matched THz frequencies for longer crystals. The effects of phase mismatch on efficiency and generated $\mathrm{THz}$ waveform are amplified for thicker crystals.

For $\mathrm{THz}$ optical rectification, frequencies $\omega_{1}$ and $\omega_{2}$, within the bandwidth of a single optical or near-infrared pump pulse, are very nearly equal in comparison to $\omega_{T H z}$. Therefore, the degree to which phase matching is satisfied is typically quantified by comparing the group velocity of the pump pulse to phase velocities of the $\mathrm{THz}$ frequency modes. The crystal length over which phase matching is approximately satisfied is quantified by the coherence length,

$$
l_{c}\left(\lambda_{\text {Pump }}, \Omega_{T H z}\right)=\frac{\pi C}{\Omega_{T H z}\left|n_{g}-n_{T H z}\right|},
$$

where $n_{g}=n_{\text {Pump }}-\lambda_{\text {Pump }} \partial n_{\text {Pump }} / \partial \lambda_{\text {Pump }}$. Specifically, the coherence length represents the length at which the accumulated phase difference between the pump pulse and a THz- 
frequency mode is equal to $\pi / 2$. It is fundamentally clear that phase matching can be satisfied by adjusting the pump frequency. In birefringent crystals the extraordinary index of refraction is angle dependent and the phase mismatch is tunable with crystal orientation [74]. Temperature tuning of $n_{P u m p}$ and $n_{T H z}$ may also be applied in some materials to achieve nonlinear phase matching [74].

Phase matching is an important consideration when choosing a crystal for optical rectification. To clearly demonstrate this importance, the coherence length of three semiconductors, discussed in Section 1.2, is mapped in Figure 2.2. The coherence length was calculated at each pump photon energy and $\mathrm{THz}$ frequency using published dispersion relations [81-85]. These calculations show the potential THz bandwidth of efficient generation, for crystals of four thicknesses, as a function of pump photon energy/wavelength. The color scheme emphasizes the potential of thin $(0.15 \mathrm{~mm})$ crystals for broadband phase matching: the red region represents a coherence length $>0.15 \mathrm{~mm}$ while the dark blue regions show negligible coherence length. Phase-matching is interrupted by strong phonon resonances at $5.4 \mathrm{THz}$, 10.96 THz, and 8.02 THz for $\mathrm{ZnTe}, \mathrm{GaP}$, and $\mathrm{GaAs}$ respectively. Similar calculations are presented for comparison in Chapter 4 for chalcopyrite structured semiconductors for which optical/near-infrared and $\mathrm{THz}$ dispersion relations are available in the literature.

ZnTe is commonly used for both generation and electro-optic detection of $\mathrm{THz}$ pulses due to its good phase matching in thin crystals at $800 \mathrm{~nm}$ from about 0.5-3.5 THz [81]. ZnTe also exhibits relatively large nonlinear coefficients. While GaP shows significantly smaller secondorder nonlinear coefficients, it is attractive due to its potential for broad-bandwidth generation (0.1-7 THz) and electro-optic detection using thin crystals $(0.15 \mathrm{~mm})$ near $800 \mathrm{~nm}$ [86]. GaP also shows good phase matching for thicker crystals near $1 \mu \mathrm{m}$, making it attractive for fiber laser pumped THz sources $[37,39]$. Among semiconductors, GaAs shows large nonlinear coefficients and exhibits the best collinear phase-matching achievable at the laser line of $1550 \mathrm{~nm}$ to date [38]. However, two-photon absorption (2PA) severely diminishes the scalability of efficiency with pump intensity at this wavelength. 

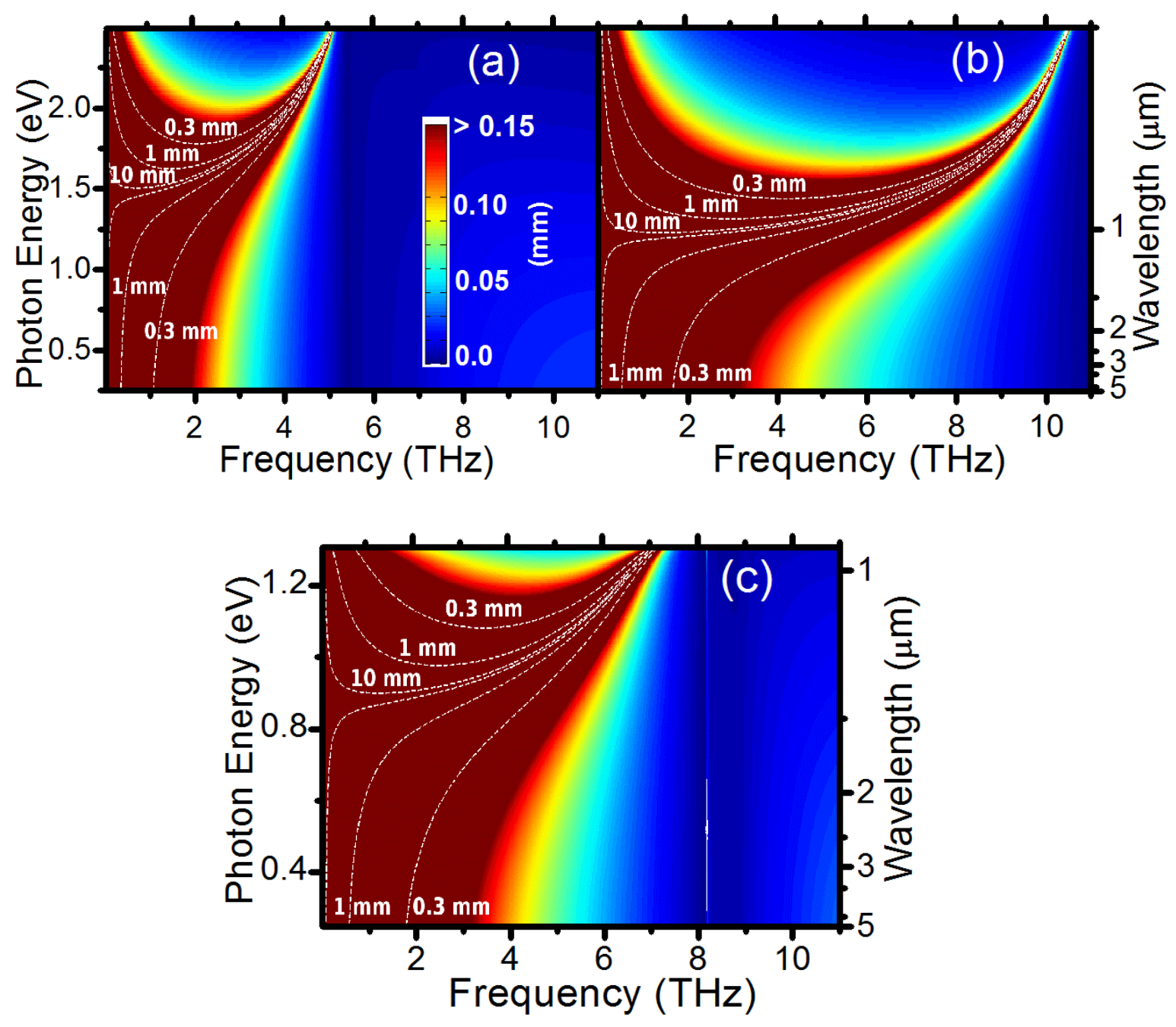

Figure 2.2: Calculated coherence lengths for (a) $\mathrm{ZnTe}$, (b) GaP, and (c) GaAs. In the red region the coherence length is greater than $0.15 \mathrm{~mm}$. Two labeled dotted lines outline regions of coherence length greater than 0.3 $\mathrm{mm}, 1.0 \mathrm{~mm}$, and $10 \mathrm{~mm}$. The darkest blue regions show negligible coherenece length.

\subsubsection{Multiphoton Absorption \& Free-Carrier Absorption}

Multi-photon absorption (MPA) has found practical applications in areas such as multiphoton spectroscopy, nonlinear optical microscopy, microfabrication and lithography, as well as optical limiting [87]; however, nonlinear absorption is detrimental to the efficiency of frequency conversion by nonlinear-optical, parametric interaction. When non-negligible, MPA tends to deplete the pulsed beam(s) pumping a nonlinear crystal. For $\mathrm{THz}$ optical rectification 
specifically, free carriers generated by multi-photon excitation across the band-gap tend to also deplete the generated $\mathrm{THz}$ pulse through free-carrier absorption (FCA). With photon energies on the order of a few meV, THz radiation is particularly prone to FCA resulting from low-energy intraband conduction band excitations. The effects of MPA are therefore an important factor affecting the intensity scalability of $\mathrm{THz}$ optical rectification and will vary from material to material.

At high pump intensities the probability of multiphoton absorption (MPA) events is enhanced considerably. Linear absorption and additional MPA is modeled by a generalized version of Beer's law:

$$
\frac{d I}{d t}=-\alpha I-\beta I^{2}-\gamma I^{3}-\ldots
$$

In this general form, $\alpha$ is the usual linear-absorption coefficient, $\beta$ is the 2PA coefficient, and $\gamma$ is the three-photon absorption (3PA) coefficient. Just as single photon absorption is strongly enhanced for photon energies greater than the band-gap of a semiconductor or dielectric, 2PA and 3PA are enhanced for photon energies greater than $1 / 2$ and $1 / 3$ of the band-gap respectively, because the energies of two/three photons must combine to promote an electron across the band-gap.

Some semiconductors, such as GaP exhibit best phase matching for photon energies below the onset of 2PA. While this condition is preferable, the effects of 2PA can be avoided or mitigated in semiconductors not exhibiting this ideal. 2PA in GaAs, which exhibits considerably stronger nonlinearity than GaP, can be avoided using quasi-phase matching with an appropriately chosen period [36]; for example, quasi-phase matched $\mathrm{THz}$ generation has been demonstrated for a fiber laser emitting at $2 \mu \mathrm{m}$ [45], well above the onset wavelength of 2PA. In this method the temporal walk-off between $\mathrm{THz}$ frequencies and pump pulse is periodically reversed by alternating the sign of the nonlinear susceptibility. Wide-area pumping has also been employed to reduce the effects of $2 \mathrm{PA}$ in $\mathrm{ZnTe}[34,35]$ by reducing the peak intensity material damage threshold. However, wide aperture semiconductors are expensive and not all semiconductors may be periodically poled. 
The higher probability of MPA events at high intensities can be understood by considering the quantum mechanical uncertainty relation $\Delta E \Delta t \geq \hbar / 2$. For any optically excited transition between real energy levels (eigenstates), energy conservation must be strictly satisfied. However, violations of energy conservation for intermediate transitions, occurring on a time scale less than the observation time $\Delta t \approx \hbar / \Delta E$, are consistent with the uncertainty principle; that is, the system may be excited to a virtual energy level (noneigenstate) for times less than $\Delta t$ (on the order of $10^{-15}-10^{-16} \mathrm{~s}$ for visible and near-infrared photon energies). $\Delta E$ is the difference in energy between a virtual energy level and the nearest eigenstate [74]. If the sum of the interacting photon energies is equal to the energy gap between eigenstates, and the transition is allowed by symmetry selection rules [88], a transition from one eigenstate to another is possible. If the necessary photons do not all simultaneously scatter within the virtual-state lifetimes the system relaxes back to the ground state. Multiple photons may alternately scatter parametrically back to the ground state; see Section 2.1.1. As a result of the strict time constraints for photon interaction, higher-order absorption events require increasingly high photon number densities to show significant probability, as is the case for the parametric scattering events described in Section 2.1.1.

2PA and 3PA scale as the third and fifth power of the electric field respectively and are classified as third-order and fifth-order nonlinear interactions. Analogous to the linear susceptibility, the real and imaginary parts of higher-order susceptibilities describe nonlinear dispersion and nonlinear absorption respectively. These higher-order susceptibilities may be derived from perturbation theory $[74,75]$ and are related to the coefficients in (2.22) by [80]

$$
\beta=\frac{3 \pi}{\varepsilon_{o} n^{2} c \lambda} \operatorname{Im}\left(\chi_{x x x x}^{(3)}\right) \text { and } \gamma=\frac{5 \pi}{\varepsilon_{o}^{2} n^{3} c^{2} \lambda} \operatorname{Im}\left(\chi_{x x x x x x}^{(5)}\right)
$$

\subsection{Terahertz Detection}

Methods of detecting $\mathrm{THz}$ pulses may be broken into two categories. In the first, absorption of the electromagnetic field (heating) is detected. For example, calibrated bolometers use the temperature-dependent resistance of a material to determine the time- 
integrated power of radiation absorbed. Well calibrated thermal detectors provide an accurate measure of the power but give no information concerning the time-domain/spectral properties of a $\mathrm{THz}$ pulse. In the second detection category, ultrafast pulses, useful for generation of $\mathrm{THz}$ radiation by any of the methods mentioned in Section 2.1, are conveniently applied for highresolution time-domain detection of $\mathrm{THz}$ pulses. Using a pulsed probe, the amplitude and phase of the THz field is detected and the absolute electric-field amplitude may be estimated. Measurement of the phase and relative amplitude of the $\mathrm{THz}$ pulse is generally well suited for practical applications: the real and imaginary parts of the dielectric constant may be simultaneously determined without resorting to a Kramers-Kronig analysis. Pulsed time-domain detection is, therefore, a powerful method applied in $\mathrm{THz}$ time-domain and time-resolved $\mathrm{THz}$ spectroscopy setups.

The two most common detection methods applying ultrafast probe (or gate) pulses are electro-optic (EO) sampling and photoconductive sampling. In both methods a probe pulse is scanned relative to the detected $\mathrm{THz}$ pulse; their relative time delay is adjusted within a material exhibiting a measureable interaction proportional to the $\mathrm{THz}$ electric-field amplitude. In the method of EO sampling, alteration of the probe-pulse polarization state, in proportion to the electric-field amplitude, due to the electro-optic effect is detected. In the method of photoconductive sampling, the electric field of the THz pulse accelerates free-carriers excited by the probe pulse generating a current density proportional to the field amplitude. Publications by Park et al. and Cai et al. [89-91] compared the efficiency, bandwidth, and signal-to-noise ratio of detection by EO sampling and photoconductive antenna. Based on their results the signal-to-noise ratio and sensitivity is superior using antenna detection below $3 \mathrm{THz}$, but potential for broadband detection is considerably higher using EO detection. Otherwise the two methods show similar performance. EO sampling was applied to obtain the experimental results summarized in later chapters; further discussion of antenna detection is therefore neglected. 


\subsubsection{Electro-0ptic Sampling}

In the standard electro-optic (or Pockels) effect a DC electric field induces a birefringence or alters an innate crystal birefringence. In this approach the $\mathrm{THz}$ field amplitude plays the role of a DC electric field; that is, for good velocity matching between a THz and probe pulse, within an EO crystal, a fixed relative time delay $\Delta t$ is maintained between the pulses and the probe pulse experiences a constant electric field amplitude $E_{T H z}(\Delta t)$. Ultrafast probe pulses of $100 \mathrm{fs}$ or less provide good resolution of the time-domain electric field amplitude for standard single cycle $\mathrm{THz}$ pulses with 1-2 ps durations. As will be clarified, the birefringence experienced by the probe varies linearly with the $\mathrm{THz}$ field amplitude $\Delta n \propto E_{T H z}(\Delta t)$ and alters the initially linear probe polarization state.

A standard setup for measuring $E_{T H z}(\Delta t)$ by EO sampling is summarized in Figure 2.3. When no overlap exists between the probe and $\mathrm{THz}$ field, the probe experiences no

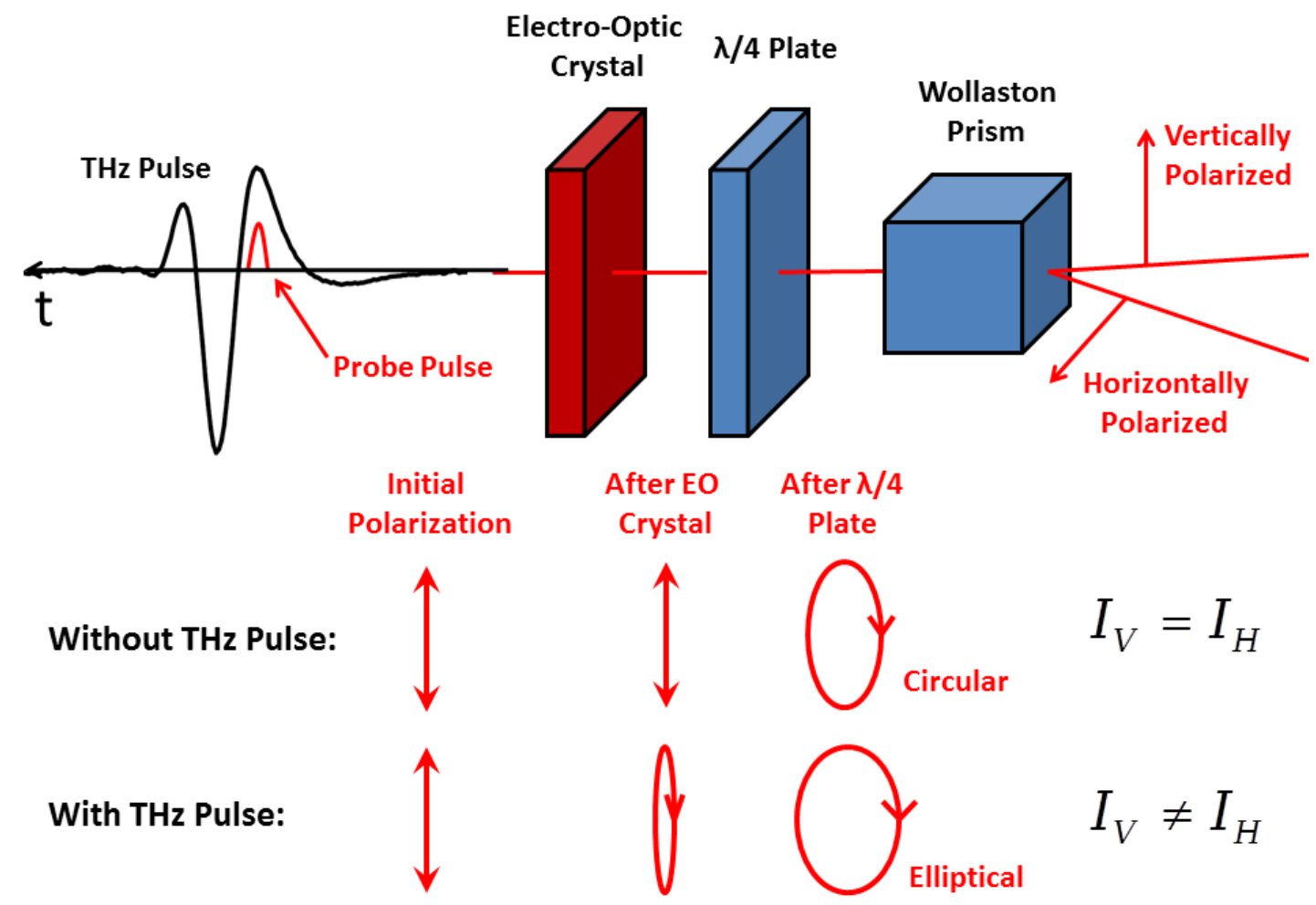

Figure 2.3: Representation of a standard electro-optic sampling setup. The polarization state is shown after each optical element with and without overlap between the probe pulse and the $\mathrm{THz}$ pulse. 
birefringence and is unaltered in passing through the EO crystal. The combination of a quarterwave plate and a Wollaston prism break the probe pulse into two orthogonal linearly polarized components of equally balanced intensity. In the presence of a $\mathrm{THz}$ electric field a delay-varying birefringence is induced. The birefringence results in a slight ellipticity of the transmitted probe polarization state: the quarter-wave plate and Wollaston prism no longer produce balanced vertical and horizontal polarization intensities. The intensity imbalance at the detectors is proportional to the induced birefringence, which is proportional to the local $\mathrm{THz}$ field experienced by the probe pulse:

$$
\Delta I(\Delta t)=I_{o} \frac{\omega}{C} \Delta n(\Delta t) L \propto E_{T H z}(\Delta t) .
$$

The difference between the vertical and horizontal polarization components $(\Delta I)$ is detectable using balanced photodetectors; thus, the relative electric field amplitude is measured as a timevarying voltage.

As mentioned in Section 2.1.1.1, EO sampling is closely related to optical rectification. The close relation is more readily apparent in comparing the second-order polarization for the EO effect,

$$
P_{i}^{(2)}(\omega)=2 \sum_{j, k} \varepsilon_{o} \chi_{i j k}^{(2)}(\omega ; \omega, 0) E_{j}(\omega) E_{k}(0),
$$

to that of optical rectification [73]

$$
P_{i}^{(2)}(0)=\sum_{j, k} \varepsilon_{o} \chi_{i j k}^{(2)}(0 ; \omega,-\omega) E_{j}(\omega) E_{k}^{*}(\omega)
$$

Both show a similarly complicated orientation dependence on both the $\mathrm{THz}$ and probe pulse polarization directions. Equation (2.25) can be rewritten to emphasize the effective alteration of the susceptibility tensor by defining a field-induced susceptibility tensor:

$$
\chi_{i j}^{(2)}(\omega)=2 \sum_{k} \chi_{i j k}^{(2)}(\omega ; \omega, 0) E_{k}(0) \quad \Rightarrow \quad P_{i}^{(2)}(\omega)=\sum_{j} \varepsilon_{o} \chi_{i j}^{(2)}(\omega) E_{j}(\omega) .
$$

In general, $\chi_{i j k}^{(2)}(0 ; \omega,-\omega) \approx \chi_{i j k}^{(2)}(\omega ; \omega, 0)$; that is, optical rectification and the EO effect share the same second-order susceptibility tensors. Equation (2.27) shows that an applied DC field alters 
the effective susceptibility tensor resulting in birefringence. Also, the optimal orientation of the $\mathrm{THz}$ and probe fields depends strongly on the crystal symmetry.

ZnTe is one of the few crystals exhibiting good broadband EO phase matching of $\mathrm{THz}$ frequencies for an $800 \mathrm{~nm}$ probe pulse. Planken et al. [92] measured and calculated the relative EO detection efficiency in ZnTe as a function of orientation. They found the (110) plane to be the most efficient plane and analyzed the angular dependence at normal THz/probe incidence to this plane. For $\alpha$ and $\varphi$ representing the linear polarization direction of the $\mathrm{THz}$ and pump pulses with respect to the crystal z-axis respectively, and assuming a small field-induced ellipticity, the dependence in the (110) plane is found to be:

$$
\Delta I(\alpha, \varphi)=I_{o} \frac{\omega n^{3} E_{T H z} r_{41} L}{2 c}(\cos \alpha \sin 2 \varphi+2 \sin \alpha \cos 2 \varphi)
$$

Here, $I_{o}$ is the probe intensity, $\omega$ is the probe frequency, $n$ is the optical/near-infrared index of refraction in the absence of an applied field, $L$ is the crystal length, and $r_{14}$ is the EO

coefficient. For $\mathrm{ZnTe}$, with $\overline{4} 3 m$ point group symmetry, all nonzero EO tensor elements are equal. Equation (2.28) indicates that the maximum EO signal is obtained when $\varphi=\alpha+\pi / 2$ or $\varphi=\alpha$ with $\alpha=\pi / 2 \pm n \pi$, where $n$ is an integer. For $\varphi=\pi / 2$ the EO detection efficiency is proportional to $\sin \alpha$; thus, in this orientation the detection crystal itself may be used to measure the $\mathrm{THz}$ polarization component along the direction of the probe pulse (i.e. the direction perpendicular to the z-axis). This method of polarized detection was used in the experiments summarized in later chapters to measure the $\mathrm{THz}$ polarization in the lab frame. The crystal and polarization were rotated to measure THz components emitted parallel (pump and probe polarizations parallel) and perpendicular (probe perpendicular to pump polarization) to the vertically polarized pump pulse.

\subsection{Experimental Setup}

A simplified version of the experimental setup, used for all experiments in later chapters, is shown and summarized in Figure 2.4 (a). The Ti:sapphire laser amplifier operating at a central wavelength of $800 \mathrm{~nm}, 1-\mathrm{KHz}$ repetition rate, and average power of 3.5 Watts 
provides 80 -fs pulses as verified by intensity autocorrelation. To achieve high peak pulse intensities, this system applies chirped-pulse amplification [93]: separate gratings are used to stretch (chirp) the "seed" pulse before amplification, in a Ti:sapphire gain medium, and compress the pulse post-amplification. Chirped-pulse amplification avoids the detrimental effects of self-phase modulation and/or laser induced damage of the gain medium by lowering the peak field intensity of the pulse during amplification. The amplifier contains two lasers, one generating $<100 \mathrm{fs}$ seed pulses at $800 \mathrm{~nm}$ and a $80 \mathrm{MHz}$ repetition rate and another generating nanosecond pulses at $527 \mathrm{~nm}$ and a $1 \mathrm{kHz}$ repetition rate. The latter laser excites the necessary gain medium population inversion for amplification of a seed pulse. The output pulse chirp/compression is continuously adjustable in our system and the pulse duration is monitored using a home-grown intensity autocorrelator; see the following section. The laser amplifier output is split, 50/50, and used for both EO sampling of generated THz pulses and pumping of a two-stage optical parametric amplifier (OPA).

The OPA generates $\sim 80-120 \mathrm{fs}$ pulses at a $1 \mathrm{kHz}$ repetition rate and average power of 100-400 mW. The output consists of two separable beams. The "signal" beam is tunable from 1.12 to $1.62 \mu \mathrm{m}$ and the "idler" is tunable from 1.62 to $2.5 \mu \mathrm{m}$. In the OPA, the $800 \mathrm{~nm}$ input pulses are split for parametric amplification (i.e. seeded difference-frequency generation) in two beta barium borate (BBO) crystals in the pre-amplification and second amplification stages. In pre-amplification, using a small fraction of the pump beam, difference-frequency generation is seeded with a broadband white-light source produced by continuum generation in a sapphire plate. Wavelength tuning is achieved by adjusting the delay of the white-light pulse with respect to the pre-amplification pump pulse and/or adjusting the crystal angle to alter the optimal phase-matched wavelength. The pre-amplification stage generates intense seed pulses for the second amplification stage, which is tuned and optimized by adjusting the second BBO crystal angle and second pump delay. The OPA employs computer-controlled translation and rotation stages for fast, repeatable, and precise optimization and recall of delays and crystal angles when tuning the output wavelength. Tuning at each wavelength was verified by observing second-harmonic generation of the OPA output in a BBO crystal with a spectrometer operating in the visible. The distance from the OPA to the zincblende/chalcopyrite sources was 

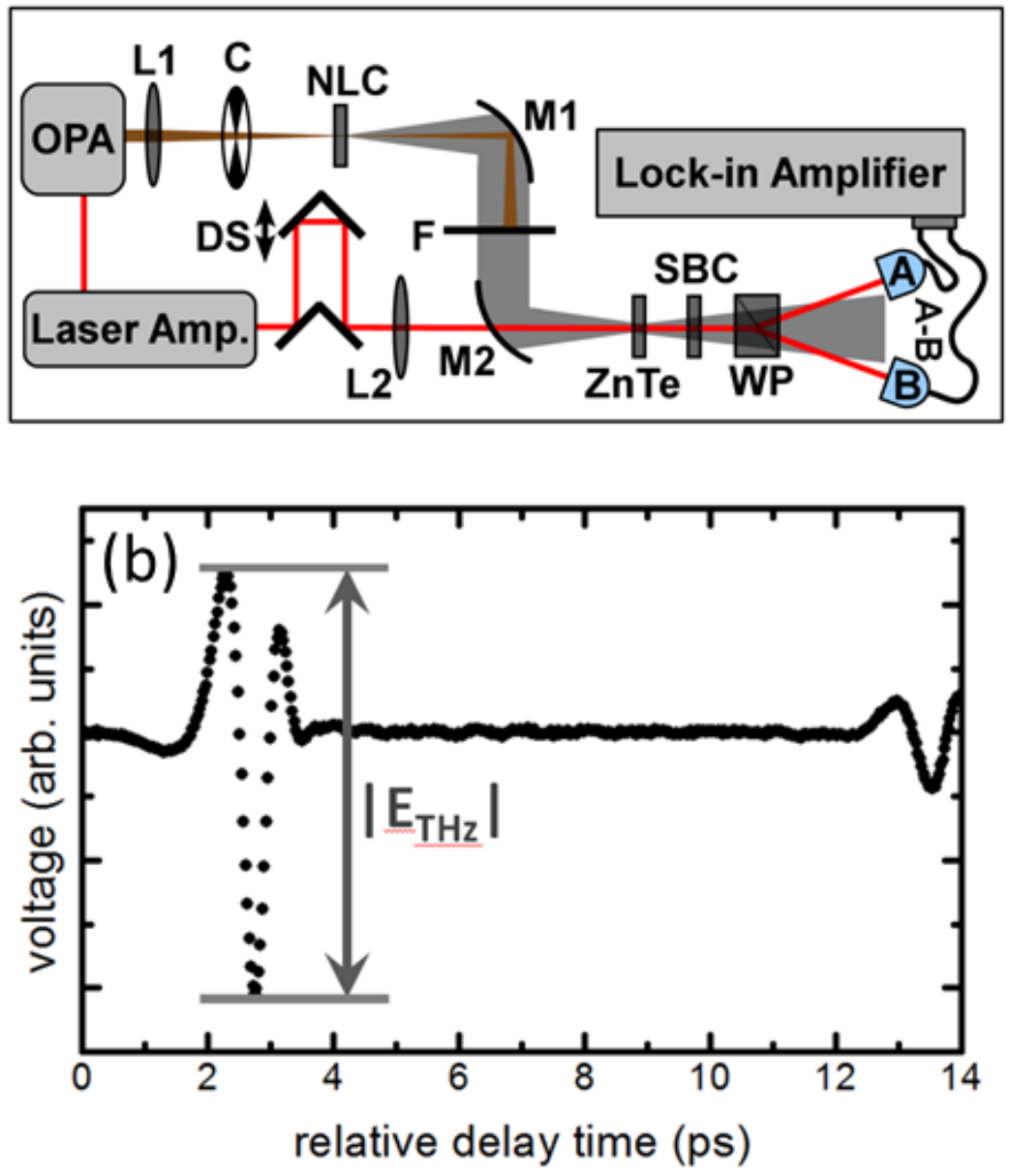

Figure 2.4: Part (a): Experimental setup (simplified). Part (b): Typical measurement of the relative THz electric field amplitude (proportional to voltage) as a function of time. The field at $\sim 14 \mathrm{ps}$ is due to internal reflection in the ZnTe EO crystal.

reduced to $<0.5$ meters to minimize effects of walk-off of the signal and idler beams with tuning of the OPA wavelength.

Referring to Figure 2.4, the output of the OPA is chopped (C) and focused (L1) into a nonlinear crystal (NLC). Due to diffraction the $\mathrm{THz}$ radiation is emitted as a frequency dependent cone. This radiation is collected and refocused into the electro-optic crystal (ZnTe) by off-axis parabolic mirrors (M1 and M2). The pump beam is filtered with a thin, black, highdensity-polyethylene filter (F). The probe is sent through a delay stage (DS) and focused into the electro-optic crystal. The THz signal is detected by EO sampling using a Soleil-Babinet compensator (SBC), Wollaston polarizing prism (WP) and two balanced photodiodes (A,B), as 
discussed in the previous section. The lock-in amplifier detects the voltage difference from photodiodes $A$ and $B(A-B)$.

Time-domain measurement of the THz electric field is achieved by EO sampling in a (110)-cut $0.5 \mathrm{~mm}$ ZnTe crystal. The (110) plane was chosen for best detection efficiency and the probe polarization, at $90^{\circ}$ to the crystal z-axis, was oriented for detection of $\mathrm{THz}$ field components parallel or perpendicular to the vertically polarized pump pulse [92]; see Section 2.2.1. For all experiments the pump beam was focused after the source to avoid damage and excessive diffraction of low-frequency $\mathrm{THz}$ field components. For repeatability when switching between samples, the back face of each sample was referenced to a fixed razor blade without altering the alignment of the setup. When necessary, the same straight edge on a micrometer translation stage was used to measure the spot-size at the source by the "knife-edge" method [94]. Experiment specific parameters and conditions are provided in later appropriate chapters.

Figure 2.4 (b) shows a standard approximately half-cycle $\mathrm{THz}$ pulse detected by EO sampling in the $0.5 \mathrm{~mm}$-thick ZnTe crystal. The pulse is slightly distorted due the limited EO detection bandwidth resulting from phase mismatch at high $\mathrm{THz}$ frequencies: as shown in Figure 2.2 (a), the phase-matching condition is only satisfied in thin crystals of ZnTe, at $800 \mathrm{~nm}$ (1.55 eV) pumping, out to about $\sim 3 \mathrm{THz}$. The efficiency of detection beyond $3 \mathrm{THz}$ is dramatically reduced altering the measured time-domain waveform. Other factors such as frequency-dependent diffraction at the source and frequency-dependent focusing at the detector affect the measured $\mathrm{THz}$ waveform to a lesser degree [78].

Home-grown VIs for LabVIEW software were written to automate data collection by interfacing with the time-delay motion controller and lock-in amplifier for signal readout. The software was configured and programmed with adjustable measurement parameters such as translation-stage step distance (i.e. time increment between data points), the total time interval sampled, the number of $\mathrm{THz}$ traces to be averaged (signal-to-noise reduction), and the relative time at which to "park" the translation stage. Lock-in amplifier settings were also programmed for remote adjustment. The collected data were automatically saved to a specified folder and filename with files continuously updated for each collected data point. 


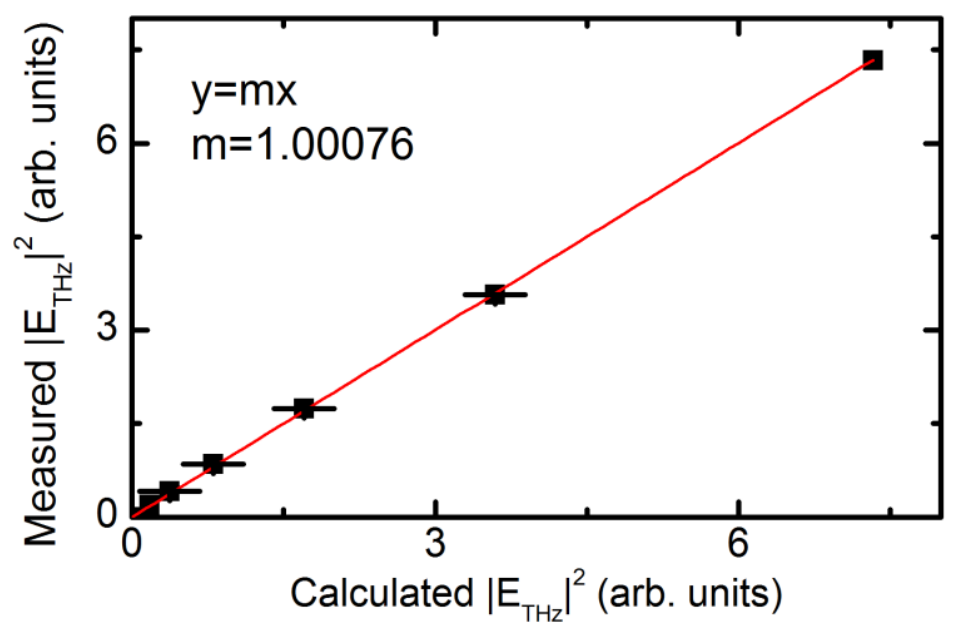

Figure 2.5: THz signal with 0-5 silicon wafers inserted into the $\mathrm{THz}$ path plotted against calculated signals based on a transmissivity of 0.49 .

A linear response of the balanced EO detection was verified by successive insertion of up to five high-resistivity silicon wafers into the $\mathrm{THz}$ beam. The measured peak-to-peak signal is plotted as a function of calculated $\mathrm{THz}$ signal is shown in Figure 2.5. The transmitted $\mathrm{THz}$ electric field amplitude was calculated using known values for the index of refraction and minimal FCA of high resistivity silicon [95]. Constant probe intensity was maintained. The highest signal data point corresponds to the insertion of no wafers. The resulting fit shows the very exact linear dependence between the measured lock-in amplifier voltage and the generated THz peak-to-peak electric field amplitude.

Black high-density polyethylene (HDPE) was chosen as a long Pass filter (F). In transmission measurements black HDPE showed a 30\% higher field-amplitude transmissivity at $1 \mathrm{THz}$ than silicon (0.5 mm thick) and a polyvinyl chloride (PVC) strip (0.3 mm thick). Also, while silicon is a standard long-pass THz filter for an $800 \mathrm{~nm}$ pump [73], it is transmitting at longer near-infrared wavelengths greater than $\sim 1.1 \mu \mathrm{m}$. 


\subsubsection{Intensity Autocorrelator}

A noncollinear intensity autocorrelator was constructed with automated data collection for characterization of laser-amplifier and OPA pulse durations. In this general approach, single pulses are split in two with a 50/50 power ratio using a beamsplitter. Copies of the original pulse are overlapped in time and space in a detection medium exhibiting a measurable nonlinear intensity-dependent optical response. The intensity autocorrelation is extracted by adjusting the relative time delay between transmitted and reflected pulse copies and from this autocorrelation the pulse duration is readily extracted. Discussion here is limited to autocorrelation by noncollinear second-harmonic generation (SHG) in a BBO crystal, exhibiting a quadratic Intensity response.

Figure 2.6 (a) shows a standard approach for obtaining an intensity autocorrelation with focused beams. The noncollinear geometry allows detection of a background free autocorrelation in the time domain. To measure the autocorrelation, split copies of a pulse, following separate paths, are tightly focused into a nonlinear crystal at some full angle $\varphi\left(\sim 10^{\circ}\right)$ and overlapped in time and space. For good time-domain resolution in this focused noncollinear pump geometry the transverse spot sizes must be significantly smaller than the product of pulse duration with the speed of light: $\tau C$. The nonlinear crystal is angle tuned for phase-matched SHG; thus, the second-harmonic beam is generated with a distinct wavevector $\mathbf{k}_{3}=\mathbf{k}_{1}+\mathbf{k}_{2}$ such that the fundamental beams are easily spatially blocked. Because SHG is a second-order nonlinear effect the generated intensity is proportional to $E_{1}\left(t_{1}\right) E_{2}\left(t_{2}\right)$, or $E(t) E\left(t-t^{\prime}\right)$ for identical pulses and a single adjustable time delay. A slow detector measures the integrated intensity of this signal:

$$
S\left(t^{\prime}\right) \propto \int_{-\infty}^{\infty}\left|E(t) E\left(t-t^{\prime}\right)\right|^{2} d t \propto \int_{-\infty}^{\infty} I(t) I\left(t-t^{\prime}\right) d t .
$$

Due to the necessity of tight focusing this method becomes problematic for high-energy laseramplifier sources producing pulse energies orders of magnitude greater than ultrafast laser oscillators. The average power of lasers oscillators and amplifiers are often comparable, but amplifier repetition rates are generally orders of magnitude lower. The resulting high peak power of individual pulses means that the pump intensity in the nonlinear crystal must be 
lowered to avoid exceeding the detection medium damage threshold and/or pulse distortion due to self-phase modulation and self-focusing. The latter effects result from an intensity dependent index of refraction at high pump fluences. These detrimental effects are readily avoided by moving to an alternate SHG geometry.

To directly measure the pulse duration for frequencies spanning the laser amplifier and

(a)

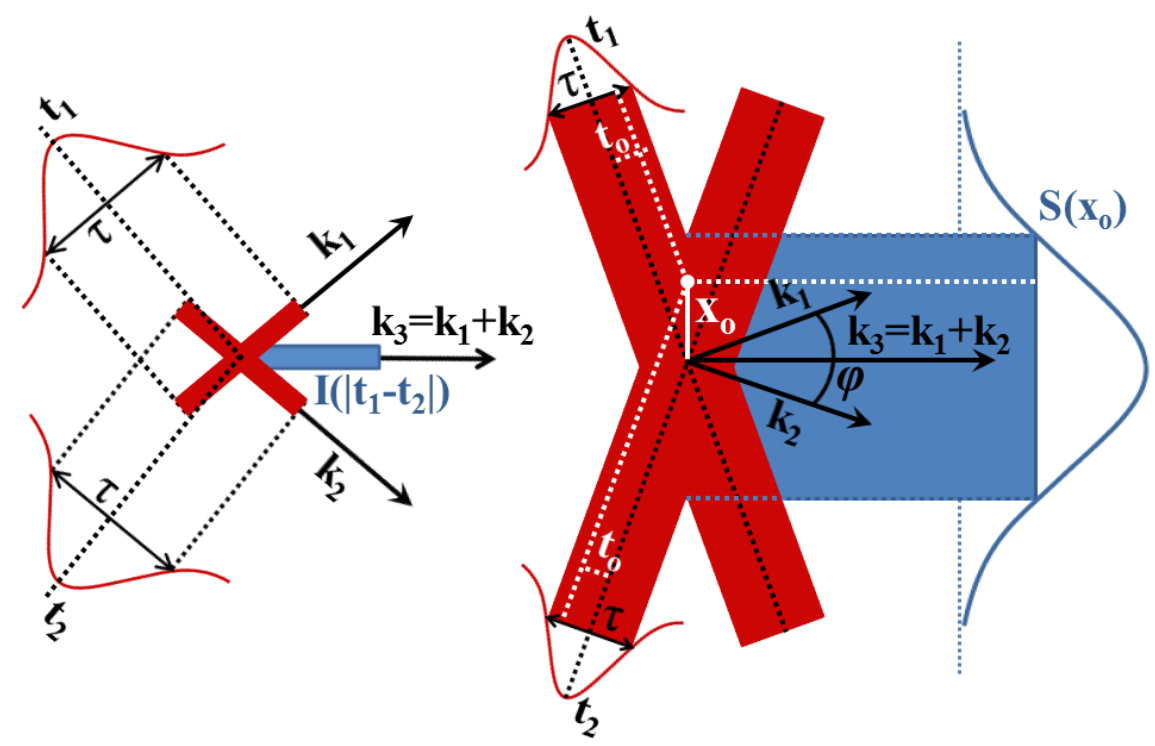

(c)

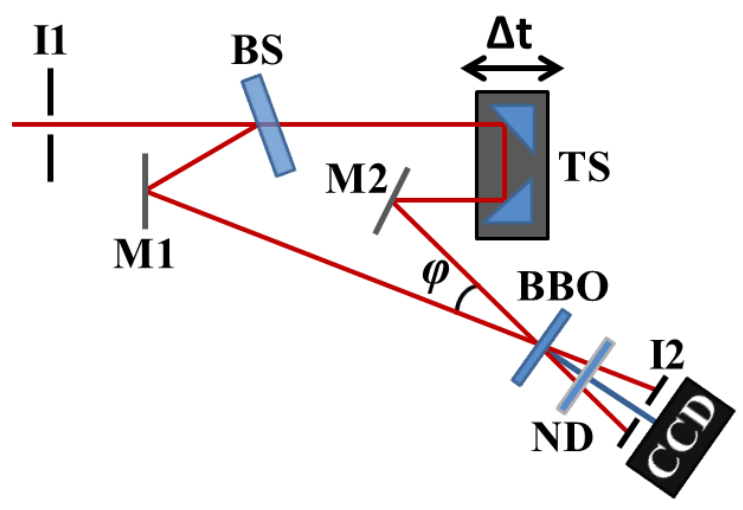

Figure 2.6: (a) Emitted SHG signal (I) as a function of time delay $\Delta t=t_{1}-t_{2}$ between two identical tightly focused pulses with duration $\tau$ and wavevectors $k_{1}$ and $k_{2}$. (b) SHG signal $S\left(x_{0}\right)$ emitted as a function of time delay $t_{0}$ across pulses of duration $\tau$. The incident pulses with wavevectors $k_{1}$ and $k_{2}$ are unfocused and large in comparison to the cross section displayed. For (a) and (b) the SHG signal is emitted with phase matched wavevector $k_{3}$. (c) Setup for the autocorrelation method in (b) showing irises (I1 and I2) for alignment, a broad bandwidth beam splitter (BS), silver mirrors (M1 and M2), translation stage (TS), a BBO crystal rotated for noncollinear SHG phase matching, and ND filter to avoid damage and saturation of the CCD array. 12 blocks the background fundamental beams and helps to minimize general light noise from the CCD detector. 
OPA tuning range, an intensity autocorrelator was constructed in an unfocused and collimated wide-beam geometry [96]. In this approach, a spatial function proportional to the intensity autocorrelation is detected. The geometry of SHG within the BBO crystal is shown in Figure 2.6 (b) (reproduced from [96]). The first and second fundamental beams must be unfocused because this approach assumes constant transverse beam intensity; an expanded beam approximates this condition. Assuming a constant transverse intensity, the transverse width and profile of the generated SHG line are related to the intensity profiles of the identical incident fundamental pulses: $I_{1}\left(t_{1}-t_{o}\right) I_{2}\left(t_{2}+t_{o}\right)$. The time delay $\left(t_{o}\right)$ across each pulse is geometrically related to the position $(x)$ transverse to the second-harmonic wavevector as

$$
t_{o}=\frac{n x_{o} \sin \varphi / 2}{C}
$$

where $\varphi$ is the full incident angle between converging fundamental beams, $n$ is the SHG medium index of refraction and $C$ is the speed of light. The SHG line profile $S\left(x_{o}\right)$ is detected using a monochrome CCD camera. As for the standard focused-beam autocorrelation, $S\left(x_{o}\right)$ is time integrated and related to the second order autocorrelation function $\left(G_{2}(t)\right)$ by:

$$
S\left(x_{o}\right) \propto \int_{-\infty}^{\infty} I\left(t+t_{o}\right) I\left(t-t_{o}\right) d t=G_{2}\left(2 t_{o}\right)
$$

From Equations (2.30) and (2.31) the actual pulse duration may be extracted without adjusting the overall time delay $\Delta t=t_{1}-t_{2}$; however, error associated with accurately measuring the angle $\varphi$ and calibrating the detector can be avoided by introducing an overall time delay between pulse copies and observing the resulting shift in SHG peak $\Delta x$. The shift is:

$$
\Delta x=\frac{c \Delta t}{2 n \sin \varphi / 2} .
$$

The shift allows for calibration of the $x$ axis, reducing error in the autocorrelation full-width half maximum (FWHM). The FWHM $\delta$ of the SHG profile along $x_{o}$ is then directly related to the FWHM fundamental pulse durations by [96]

$$
\tau=K \delta \frac{\Delta t}{\Delta x} .
$$


$K$ is a factor dependent on the assumed incident pulse shape, generally Gaussian $(K=1.414)$ or $\operatorname{sech}^{2}(K=1.55)$.

Figure 2.6 (c) shows the autocorrelator setup and optics. Processing of the CCD image, delay stage control and extraction of the pulse duration are each automated with LabView coding. For a user defined overall time delay $\Delta t$ the coded software extracts both $\Delta x$ and $\delta$ using the known CCD array pixel size. A specified number of pulse duration measurements are collected and averaged. For each measurement $\delta$ a user-specified number of CCD pixel rows parallel to $x_{o}$ are averaged. The distance between the CCD camera and BBO crystal is minimized to less than $2-3 \mathrm{~cm}$ to avoid spreading of the SHG beam due to diffraction.

\subsection{Terahertz Spectroscopy}

$\mathrm{THz}$ photons have energies on the order of a few meV. Too low in energy to bridge semiconductor band-gaps or excite common electronic transitions resulting from optical or near-infrared light-matter interactions. These low-energy $\mathrm{THz}$ photons then provide a unique electronic probe in diverse systems. For example, THz radiation is highly sensitive to low-energy intraband excitation of free carriers. This sensitivity to free-carrier absorption makes $\mathrm{THz}$ pulses an excellent probe of material conductivity and photoconductivity. Also, any materials opaque at visible and near-infrared frequencies are transparent at $\mathrm{THz}$ frequencies. $\mathrm{THz}$ transmission spectroscopy or THz TDS is then a practical and unique tool providing spectroscopic fingerprints for material identification. THz radiation is also sensitive to unique vibrational and rotational resonances in solids and molecules, allowing direct excitation and probing of these resonances.

While the experiments in the chapters to follow focus primarily on $\mathrm{THz}$ source development and characterization, the long-term purpose of the $\mathrm{THz}$ setup (Section 2.3) will be time-domain and time-resolved THz spectroscopic measurements. In this section, $\mathrm{THz}$ timedomain spectroscopy (THz TDS) and time-resolved THz spectroscopy (TRTS) are discussed and results for both methods are presented. Preliminary time-domain measurements of $\mathrm{ZnGeP}_{2}$ and published time-resolved measurements of free-carrier absorption in hematite/reduced graphine oxide are presented. 


\subsubsection{Terahertz Time-Domain Spectroscopy}

THz TDS provides fundamental frequency-dependent material parameters related to conductivity, absorption, and dispersion by careful comparison of a $\mathrm{THz}$ pulse transmitted through a sample of interest to a reference pulse. Carrier specific parameters such as carrier mobility and number density may also be determined. By applying phase-sensitive detection methods such as EO and photoconductive sampling the amplitude and phase of the $\mathrm{THz}$ electric field are collected simultaneously, rendering a Kramer-Kronig analysis unnecessary in extracting the above parameters. "Time-domain" refers to the domain in which the data is collected and does not imply that system dynamics are sampled; that is, THz TDS is only sensitive to equilibrium properties of a sample [4]. Probing of dynamic material properties will be discussed in the next subsection. The experimental setup for THz TDS is identical to that shown in Figure 2.4 with the addition of two off-axis parabolic mirrors. These provide an intermediate focus to accommodate standard sample dimensions.

The $\mathrm{ZnGeP}_{2}$ sample tested is a (110)-cut crystal with $3.01 \mathrm{~mm}$ thickness. A (012)-cut $\mathrm{ZnGeP}_{2}$ crystal was used as a $\mathrm{THz}$ source with pump pulses tuned to $1280 \mathrm{~nm}$ with known vertical polarization oriented along the projection of the $z$ axis into the crystal-cut plane; see Section 3.3. This results in $\mathrm{THz}$ emission with known linear polarization perpendicular to the pump polarization. Transmitted $\mathrm{THz}$ pulses polarized parallel (extraordinary wave) and perpendicular (ordinary wave) to the z-axis were therefore easily detected by orienting the (110)-cut $\mathrm{ZnGeP}_{2}$ sample. In general, great care must be taken to assure that the experimental conditions are identical during measurement of the transmitted and reference pulses. Because $\mathrm{THz}$ radiation is highly susceptible to water absorption, a dry nitrogen purge was applied covering the full THz path from source to detector; this eliminates water absorption lines that may exhibit time variance and introduce artifacts into the spectra. An average of five timedomain traces were collected and averaged for the reference and transmitted pulses respectively to minimize effects from intensity instability in the laser amplifier and OPA.

Measured reference and transmitted pulses are shown in Figure 2.7 (a). Addition optical path length plus reflection and absorption losses, due to the insertion of the $\mathrm{ZnGeP}_{2}$ sample, are evident in the transmitted pulse relative to the reference. These traces represent the raw 

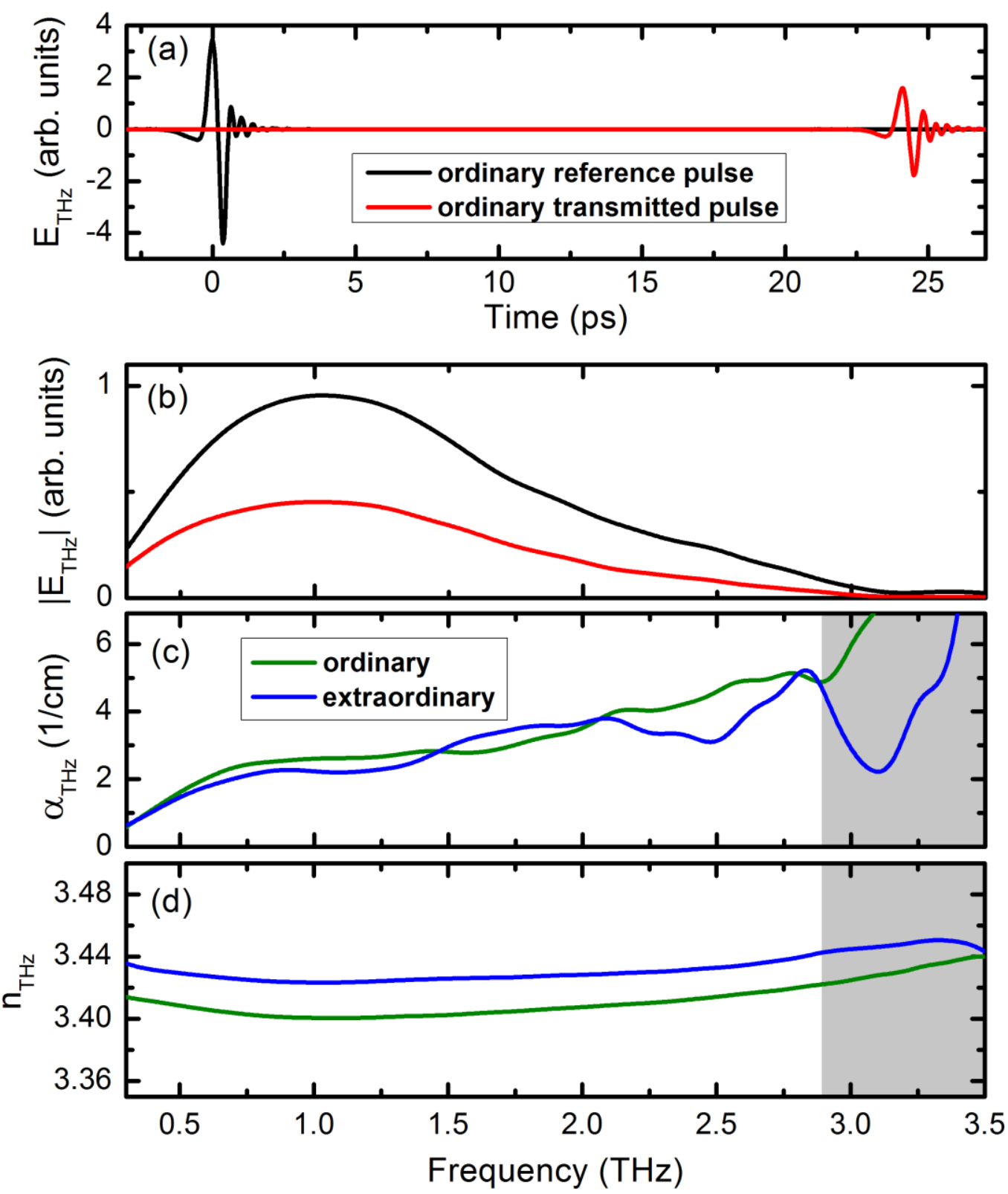

Figure 2.7: (a) Time-domain reference (black) and transmitted (red) pulses. The diminished amplitude of the transmitted pulse is due to reflection and absorption while the time delay depends on crystal thickness and dispersion. (b) Amplitude fast Fourier transforms of the time-domain traces shown in (a). (c) Calculated absorption coefficient for ordinary wave and extraordinary wave polarization. (d) Calculated ordinary and extraordinary refractive indices for $\mathrm{ZnGeP}_{2}$. The gray regions in figures (c) and (d) are show poor accuracy due to the low signals beyond $\sim 2.5 \mathrm{THz}$.

data, zero padded, such that both pulses have the same number of elements in the time domain. Figure 2.7 (b) shows amplitude fast Fourier transforms (FFT) of the pulses in (a). While the spectral amplitudes are sufficient to determine the transmissivity, the phase associated with each complex element of the FFT must be considered to determine the index of refraction. 
The goal is then to determine the real and imaginary parts of the complex index of refraction, $\tilde{n}=n-i \kappa$, from the imaginary Fourier components.

The amplitude and phase of the reference $\left(\tilde{E}_{r}\right)$ and transmitted $\left(\tilde{E}_{t}\right)$ pulses are well represented in complex form as

$$
\begin{aligned}
& \tilde{E}_{r}=E_{o} e^{i k r}=E_{r} e^{i \varphi_{r}} \\
& \tilde{E}_{t}=E_{o} \tilde{t}_{f} \tilde{t}_{b} e^{i k(r-L)} e^{i \tilde{n} k L}=E_{t} e^{i \varphi_{t}}
\end{aligned}
$$

Here, $E_{o}$ is the incident electric-field amplitude, $\tilde{t}_{f, b}$ are the amplitude transmission coefficients for the front and back crystal faces, $k=\omega / c$ is the wavevector, $r$ is the freespace path length, $L$ is the sample thickness, and $E_{r}\left(E_{t}\right)$ and $\varphi_{r}\left(\varphi_{t}\right)$ are the amplitude and phase of the reference (transmitted) complex field. The ratio of the complex fields is then

$$
\frac{\tilde{E}_{t}}{\tilde{E}_{r}}=\tilde{t}_{f} \tilde{t}_{b} e^{\alpha L / 2} e^{i(n-1) k L}=\frac{E_{t}}{E_{r}} e^{i\left(\varphi_{t}-\varphi_{r}\right)} .
$$

In Equation (2.35) the relation, $\alpha=2 k \kappa$, is applied. For samples with low absorption it is reasonable to assume that the index of refraction is real, such that the transmission coefficient is also real and defined by the Fresnel equations at normal incidence

$$
t_{f}=1-r_{f}=1-\frac{1-n}{1+n} \quad ; \quad \mathrm{t}_{b}=1-r_{b}=1-\frac{n-1}{1+n}
$$

Equation (2.35) then becomes

$$
\frac{\tilde{E}_{t}}{\tilde{E}_{r}}=\frac{4 n}{(1+n)^{2}} e^{-\alpha L / 2} e^{i(n-1) k L}=\frac{E_{t}}{E_{r}} e^{i\left(\varphi_{t}-\varphi_{r}\right)}
$$

By equating the imaginary parts in Equation (2.37), a closed-form solution for the index is obtained:

$$
n=\frac{\left(\varphi_{t}-\varphi_{r}\right)}{(2 \pi f / C) L}+1
$$

By equating the real parts a solution for the linear absorption is obtained: 


$$
\alpha=-\frac{2}{L} \ln \left(\frac{E_{t}}{E_{r}} \frac{(1+n)^{2}}{4 n}\right)
$$

The linear absorption coefficient and index of refraction of $\mathrm{ZnGeP}_{2}$ are calculated using Equations (2.38) and (2.39) and are shown in Figure 2.7 (c) and (d) for the ordinary and extraordinary waves. The data are reasonably accurate out to approximately $2.5 \mathrm{THz}$; beyond this frequency the transmitted amplitude is small and the results are questionable. The absorption increases for both waves at higher frequencies approaching the known phonon resonance at 3.6 THz (e-wave) and 4.24 THz (o-wave) [97]. The dielectric response is dominated by the attributes of these and other phonon resonances in the far-infrared. Limited accuracy in measuring the crystal thickness is the largest source of error in this preliminary measurement and analysis. The thickness was measured using a micrometer and is only accurate to the nearest hundredth of a millimeter. These preliminary results should be improved upon in future measurements by simultaneous determination of the optical constants and crystal thickness [98], using multiple crystal thicknesses to remove phase ambiguity, or applying a more complex analysis of the measured transients [99]. However, even using the above simple model, reasonable agreement (within one hundredth) with previous measured refractive index values at $1.5 \mathrm{THz}$ is observed [56].

\subsubsection{Time-Resolved Terahertz Spectroscopy}

$\mathrm{THz}$ pulses are routinely generated using ultrashort pulses with durations in the range of tens to hundreds of femtoseconds. High-resolution time-resolved spectroscopic measurements are therefore readily implemented, usually in the form of an optical pump and low-energy $\mathrm{THz}$ probe. This approach is referred to as time-resolved THz spectroscopy (TRTS). Application of this method allows time-resolved extraction of the complex photoconductivity and free-carrier density with resolution of a few picoseconds. Also, the sensitivity of THz radiation to free carrier absorption (FCA) allows for the extraction of carrier relaxation times and potential gleaning of photoexcited carrier relaxation pathways in structured materials. It therefore provides a 
powerful technique for both fundamental scientific studies and practical characterization of material systems $[6,100,101]$.

While not a central topic of this dissertation, the $\mathrm{THz}$ spectroscopy setup was expanded and modified for time-resolved measurements. The modified setup is shown in Figure 2.8 (a), with components defined in the caption. The key alterations from the setup in Figure 2.4 (a) are the addition of an intermediate focus (two additional off-axis parabolic mirrors) and an ultraviolet/optical sample pump beam with variable time delay; identical EO sampling and balanced detection are utilized. The LabView code automating the original system was modified to control and set parameters for both translations stages and save a full $\mathrm{THz}$ time-domain transient for each step of the sample pump delay stage.

The modified setup is now routinely applied to the characterization of photoexcited electron-hole relaxation times in nanomaterials. Specifically, hematite/reduced graphine oxide $\left(\alpha-\mathrm{Fe}_{2} \mathrm{O}_{3} / \mathrm{rGO}\right)$ composites were examined with distinct pump-probe techniques (time-domain THz spectroscopy and transient absorption spectroscopy) to determine the mechanism behind increased photocurrent and a reduction in charge recombination rate; these effecs lead to an enhancement of photocatalytic water oxidation activity [102]. Using these methods the decay of carriers photoexcited by a $400 \mathrm{~nm}$ sample pump was measured with a broadband $\mathrm{THz}$ pulse (0.5-3 THz) or a $700 \mathrm{~nm}$ visible light probe. Three sample types were measured for comparison: rGO, hematite nanoparticles $\left(\alpha-\mathrm{Fe}_{2} \mathrm{O}_{3}\right)$, and $\alpha-\mathrm{Fe}_{2} \mathrm{O}_{3} / \mathrm{rGO}$ composites. The $700 \mathrm{~nm}$ probe is sensitive to both trapped and free carriers; due to small photon energies $\left(k_{B} T\right.$ at room temperature) the $\mathrm{THz}$ probe is only sensitive to free carriers.

The THz probe data in Figure 2.8 (b) show the decay of mobile carriers excited in the $\alpha$ $\mathrm{Fe}_{2} \mathrm{O}_{3}$ and $\mathrm{rGO}$; the decay occurs on a picosecond time scale due to carrier trapping and coupling to acoustic and optical phonons [103-105]. The recombination dynamics in the $\alpha$ $\mathrm{Fe}_{2} \mathrm{O}_{3} / \mathrm{rGO}$ composite shows distinctive behavior from the noncomposites, with a broadened initial peak, which undergoes a two-stage decay process. The broadening indicates that mobile carriers were still being created well after the initial excitation pulse ( $100 \mathrm{fs})$ and then decayed along the rGO recombination pathways. This was only possible if the mobile carriers 

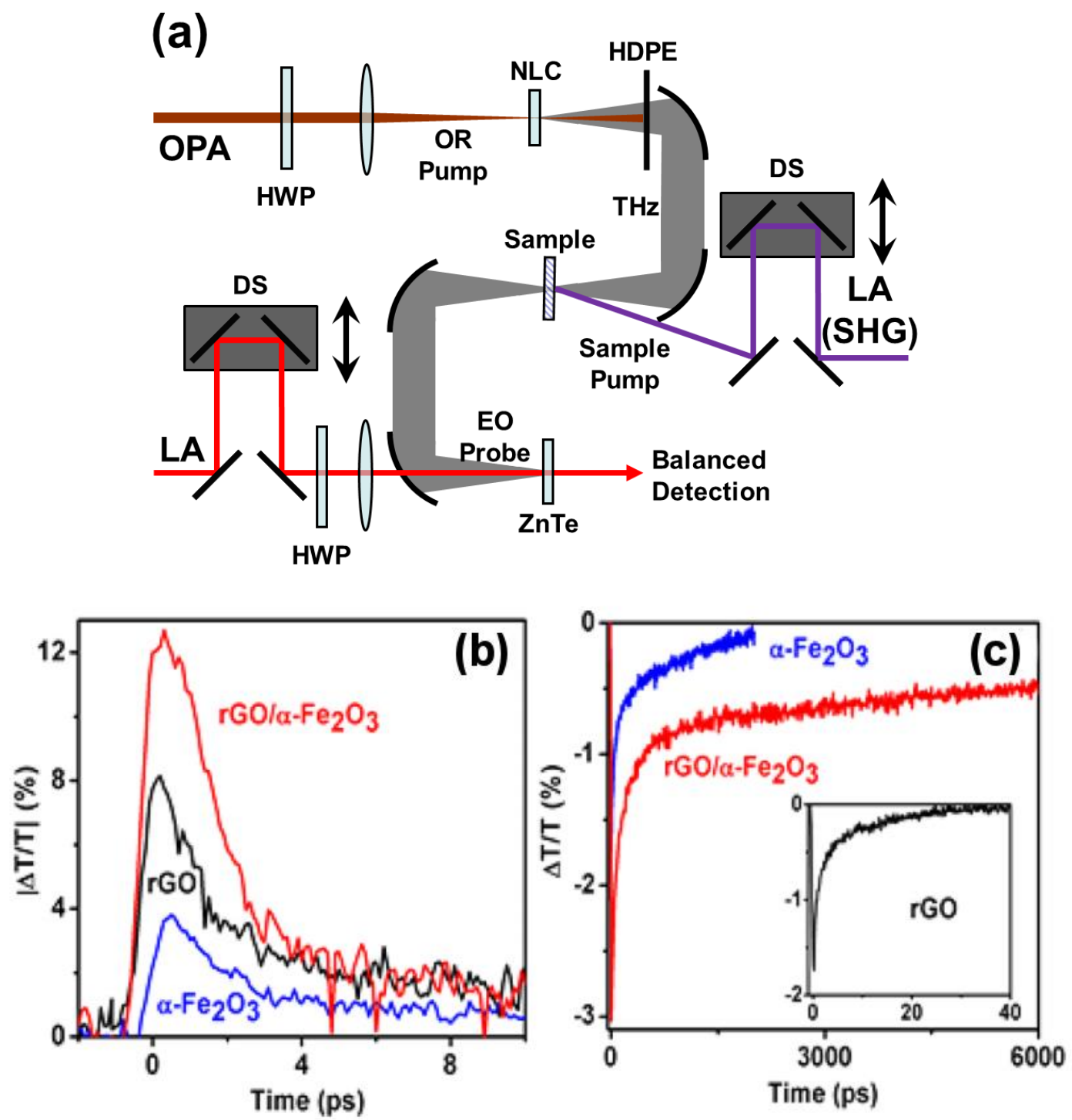

Figure 2.8: (a) Alternate setup, with an intermediate focus and additional delay stage (DS), for optical-pump THz-probe measurements. The OPA pumps a nonlinear crystal (NLC) THz source. The OR pump is filtered with a film of black HDPE and four off-axis parabolic mirrors collect and focus the emitted THz pulses. The sample of interest is placed at the intermediate $\mathrm{THz}$ focus and is pumped with ultraviolet or optical pulses with variable delay (DS). The laser amplifier (LA) provides $800 \mathrm{~nm}$ pulses for electro-optic sampling (EO Probe) in $\mathrm{ZnTe}$. Time-domain THz transients are measured with balanced detection. (a) and (b) show THz transient absorption data for a $\mathbf{4 0 0} \mathrm{nm}$ sample pump pulse. (a) shows data for a THz probe and (b) shows data for a 700 $\mathrm{nm}$ near infrared probe. The vertical axis shows the relative change in transmission.

created in the $\alpha-\mathrm{Fe}_{2} \mathrm{O}_{3}$ were being transferred to the rGO within several picoseconds of the initial excitation.

The dynamics of the transferred carriers were determined using the $700 \mathrm{~nm}$ probe and the same excitation conditions as the $\mathrm{THz}$ probe. In general, the $700 \mathrm{~nm}$ probe is sensitive to 
mobile and trapped electrons, but in $\mathrm{rGO}$ a $700 \mathrm{~nm}$ probe only interacts with electron trap states [103]. The photoexcited carriers in $\alpha-\mathrm{Fe}_{2} \mathrm{O}_{3}$ decayed within $\sim 1 \mathrm{~ns}$; see Figure 2.8 (c). The photoexcited carriers in rGO decayed quickly because of the metallic like behavior of $\mathrm{rGO}$, as shown in the inset in Figure 2.8 (c). The composite structure again differed from both the constituents. The composite structure had an electron trap state population with a decay time greater than 6 ns. This indicated that after injection of electrons into $\alpha-\mathrm{Fe}_{2} \mathrm{O}_{3}$, they quickly diffused throughout the rGO and became trapped as long-lived carriers. The trap states were caused by the structural disorder and the oxygen-containing functional groups in rGO [103]. The increased relative transmission $|\Delta T| / T$ (proportional to the number of carriers) for the composite indicates a larger charge separation rate. Interface states, between $\mathrm{rGO}$ and $\alpha-\mathrm{Fe}_{2} \mathrm{O}_{3}$ likely contributed to this increase.

\subsection{Chapter Summary}

To provide insight into the results summarized in later chapters, methods of $\mathrm{THz}$ emission and detection have been discussed in Sections 2.1 and 2.2 with focus on the specific methods studied and applied: THz optical rectification and EO sampling. Factors potentially limiting the efficiency of $\mathrm{THz}$ emission include the anisotropy of the material second-order nonlinear response, dispersion dependent phase mismatch, and intensity dependent multiphoton absorption. The optical setup, for all experiments in later chapters, has been discussed in Section 2.3 including laser systems generating the pump and probe pulses for optical rectification and EO sampling respectively; the specific mechanics of EO detection of emitted $\mathrm{THz}$ pulses and a home-grown intensity autocorrelator, applied in measure of the pump and probe pulse durations, have also been explained.

The preliminary results for THz TDS and a discussion of published results using TRTS (Section 2.4) are specific examples of the many application of $\mathrm{THz}$ spectroscopy. Intense $\mathrm{THz}$ sources are important for reduction of signal-to-noise in $\mathrm{THz}$ spectroscopy, while broadband emitters and EO crystals provide a greater spectroscopic detection bandwidth. Alternative TRTS measurements are reliant on strong sources: high pulse energy $\mathrm{THz}$ pumps can modify a medium, which can then be probed by a weak optical or $\mathrm{THz}$ pulse. The remainder of this 
dissertation explores chalcopyrite semiconductors for intense and broadband emission, properties which are significant or vital depending on the method of $\mathrm{THz}$ spectroscopy. 


\section{Chapter 3 Optical Rectification in Uniaxial Birefingent Crystals}

To date, efficient single-cycle pulses have been demonstrated by tilted-pulse-front pumping in LiNbO3 [106], collinear phase matching in GaSe [107], organic dimethyl amino 4-Nmethylstilbazolium tosylate (DAST) [108] and 2-[3-(4-hydroxystyryl)-5,5-dimethylcyclohex-2enylidene]malononitrile (OH1) [109] crystals, all of which are birefringent. In nonlinear optics in general, birefringence allows for tuning of phase-matching conditions, but also adds potential complications. To make full use of various nonlinear optical crystals for broadband $\mathrm{THz}$ generation, it is useful to explore the effects of birefringence on optical rectification in uniaxial and biaxial materials. An example of a positive-uniaxial birefringent $\mathrm{THz}$ source is $\mathrm{ZnGeP}_{2}, \mathrm{a}$ chalcopyrite crystal and ternary analog of the zincblende structure. In a direct comparison of $\mathrm{THz}$ emission by optical rectification from $\mathrm{GaP}$ and $\mathrm{GaAs}$ [46], $\mathrm{ZnGeP}_{2}$ shows promise for excitation by near-infrared sources such as pulsed-fiber or Cr:forsterite laser amplifiers ( 1240 $\mathrm{nm})$. However, the noncubic symmetry of its lattice results in significant birefringence. The work presented in this chapter explores the orientation dependence of uniaxial birefringent chalcopyrite crystals. The angular dependence of $\mathrm{THz}$ emission from birefringent crystals is found to differ significantly from that of cubic crystals. This work was performed to determine the most efficient planes for optical rectification in $\mathrm{ZnGeP}_{2}$ and to build understanding of the optical rectification process generally.

Chalcopyrite crystals provide an ideal choice for a study of the effects of birefringence due to similarity with cubic zincblende structures. Because the second-order nonlinear tensors of these two structures have identical nonzero elements, differences in the respective angular dependence result either from differing values of the nonzero tensor elements or effects due to birefringence. The experiments in this chapter provide strong evidence that the latter is highly significant. First, analysis is presented comparing the rotational dependence of $\mathrm{THz}$ emission from (110) zincblende and chalcopyrite crystals. Second, the models developed are fit to experimental data from (110) cut $\mathrm{ZnGeP}_{2}$ and $\mathrm{GaP}$ as a function of crystal rotation, indicating 
that birefringence is sufficient to describe the observed and previously unreported angular dependence. The analysis is then extended to other crystal orientations to demonstrate optimization of the effective nonlinear coefficient.

\subsection{General Analysis}

To compare the orientation dependence of $\mathrm{THz}$ optical rectification from isotropic GaP to that from birefringent $\mathrm{ZnGeP}_{2}$, it is natural to first examine the (110) plane based on the simple analysis of Section 2.1.1.1. The zincblende analysis presented here alters the approach of Section 2.1.1.1 to model the specific orthogonal electric field polarization components experimentally sampled in the lab frame. Effects of birefringence are then incorporated into the model to predict the orientation dependence of $\mathrm{ZnGeP}_{2}$. Figure 3.1 shows the pump polarization and crystal rotation at the source. The $z^{\prime}$-axis is defined by the projection of the crystal z-axis onto the crystal plane of interest; $\theta$ defines the angle between the $z^{\prime}$-axis and

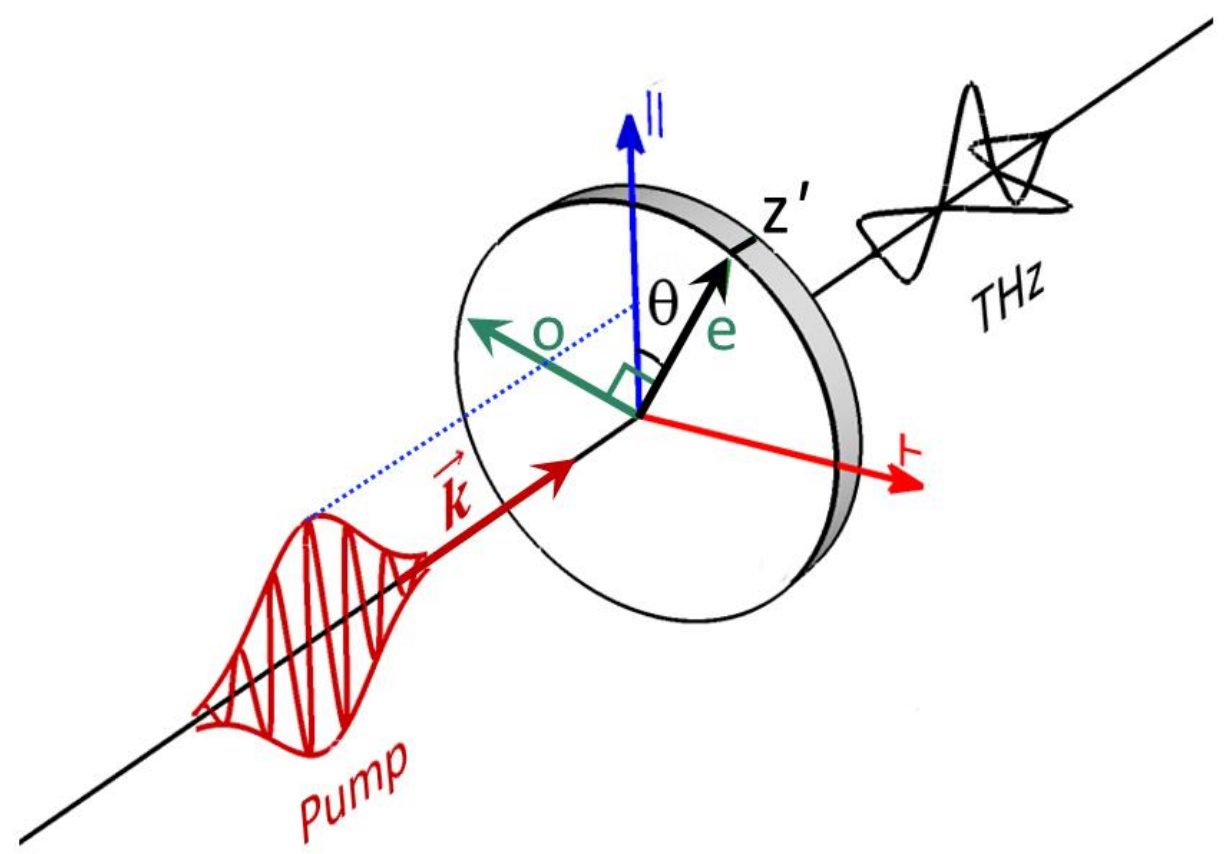

Figure 3.1: Measured polarization components of the emitted THz pulse parallel (I I) and perpendicular ( $\perp$ ) to the pump pulse polarization. Also shown is the definition of the angle $\theta$ between the pump pulse polarization and the $z^{\prime}$-axis and the axes of the ordinary and extraordinary waves (parallel and perpendicular to the $z^{\prime}$-axis respectively). 
pump polarization axis (vertical). Because it is only possible to sample the $\mathrm{THz}$ polarization along a single axis (see Section 2.2.1), a full picture of the angular dependence is obtained by measuring $\mathrm{THz}$ polarizations in two orthogonal directions: parallel $(\|)$ and perpendicular $(\perp)$ to the pump pulse polarization. Figure 3.1 also shows axes of the ordinary and extraordinary field components relevant for birefriengent crystals in which the pump polarization is split into two components inside the crystal; a component polarized parallel (perpendicular) to the $z^{\prime}$-axis experiences the extraordinary (ordinary) index of refraction. Because the pump wavevector is always directed/considered perpendicular to the planes examined, the $z^{\prime}$-axis resides in the "principle plane" containing the pump wavevector and crystal z-axis. For the (110) orientation the crystal z-axis lies within the plane and is directly referenced.

\subsubsection{Zincblende (110)}

For a pump polarization confined to the (110) plane, the optical field is represented in the crystal frame using Equation (2.17) with $\phi=\pi / 4$ :

$$
\vec{E}=\left(\begin{array}{l}
E_{x} \\
E_{y} \\
E_{z}
\end{array}\right)=E_{o}\left(\begin{array}{c}
\frac{1}{\sqrt{2}} \sin \theta \\
-\frac{1}{\sqrt{2}} \sin \theta \\
\cos \theta
\end{array}\right) .
$$

$E_{o}$ is the pump electric field amplitude and $\theta$ is the angle between the in-plane pump polarization and crystal z-axis; see Figure 3.1. The second-order polarization due to optical rectification is [73]

$$
P_{i}^{(2)}=\sum_{j, k} 2 \varepsilon_{o} d_{i j k}(0 ; \omega,-\omega) E_{j}(\omega) E_{k}^{*}(\omega),
$$

where $\varepsilon_{o}$ is the permittivity of free space, $E_{j(k)}(\omega)$ is (are) the pump electric field(s) and $d_{i j k}$ is the second-order nonlinear tensor. Applying Equation (3.2), and the contracted notation defined by (2.15), the special case of (2.18) for the (110) plane is 


$$
\left(\begin{array}{l}
P_{x}^{(2)} \\
P_{y}^{(2)} \\
P_{z}^{(2)}
\end{array}\right)=2 \varepsilon_{o} E_{o}^{2}\left(\begin{array}{c}
-\sqrt{2} d_{14} \cos \theta \sin \theta \\
\sqrt{2} d_{14} \cos \theta \sin \theta \\
-d_{36} \sin ^{2} \theta
\end{array}\right) .
$$

To determine the lab-frame components parallel and perpendicular to the initial pump polarization straightforward coordinate transformations are necessary:

$$
\begin{gathered}
P_{\|}^{(2)}=\left(\begin{array}{lll}
\frac{1}{\sqrt{2}} \sin \theta & -\frac{1}{\sqrt{2}} \sin \theta & \cos \theta
\end{array}\right)\left(\begin{array}{l}
P_{x}^{(2)} \\
P_{y}^{(2)} \\
P_{z}^{(2)}
\end{array}\right) ; \\
P_{\perp}^{(2)}=\left(\begin{array}{lll}
\frac{1}{\sqrt{2}} \cos \theta & -\frac{1}{\sqrt{2}} \cos \theta & -\sin \theta
\end{array}\right)\left(\begin{array}{l}
P_{x}^{(2)} \\
P_{y}^{(2)} \\
P_{z}^{(2)}
\end{array}\right) .
\end{gathered}
$$

After simplification the parallel and perpendicular components are

$$
\begin{gathered}
P_{\|}^{(2)}=-2 \varepsilon_{o} E_{o}^{2}\left(2 d_{14}+d_{36}\right) \cos \theta \sin ^{2} \theta ; \\
P_{\perp}^{(2)}=-2 \varepsilon_{o} E_{o}^{2}\left(2 d_{14} \cos ^{2} \theta \sin \theta+d_{36} \sin ^{3} \theta\right) .
\end{gathered}
$$

Considering the form of the nonlinear tensor as the only determining factor, Equations (3.6) and (3.7) should accurately represent the angular dependence of detected THz emission in the lab frame for a linear pump polarization confined to a (110)-cut plane in zincblende and chalcopyrite structured semiconductors. However, it will be shown that birefringence of the noncubic chalcopyrite structure plays an important role.

For the zincblende structure specifically, $d_{14}=d_{36}$ and the parallel and perpendicular components can be further simplified

$$
\begin{aligned}
& E_{T H z}^{\|} \propto P_{\|}^{(2)}=-6 \varepsilon_{o} E_{o}^{2} d_{14}\left(\cos \theta-\cos ^{3} \theta\right) ; \\
& E_{T H z}^{\perp} \propto P_{\perp}^{(2)}=2 \varepsilon_{o} E_{o}^{2} d_{14}\left(-2 \sin \theta+3 \sin ^{3} \theta\right) .
\end{aligned}
$$

\subsubsection{Chalcopyrite (110)}

In a noncubic birefringent crystal the pump pulse polarization will be broken into two components with two distinct group/phase velocities. The amplitude of these ordinary (o) and 
extraordinary (e) components depends on the incident polarization $(\theta)$ and the total field; it may be expressed as

$$
E(\theta, d, t)=E_{o} \sin \theta \exp \left[i\left(k_{o} d-\omega t\right)\right]+E_{o} \cos \theta \exp \left[i\left(k_{e} d-\omega t\right)\right]
$$

For a pump polarization confined to the (110) plane the o and e components are perpendicular and parallel to the crystal z-axis respectively, $E_{o}$ and $\theta$ are defined as previously, $\omega$ is the optical frequency, and $k_{o}$ and $k_{e}$ are the wavevectors of the o- and e-waves respectively. The polarization state at each position $z$ only depends on the relative accumulated phase between the $o$ and e waves $\left|k_{o}-k_{e}\right| d \equiv \Delta k d$; thus, the phase can be assigned to the z-component of the field without altering the analysis. The field in the crystal is then identical to Equation (3.1) with an added relative phase

$$
\vec{E}=\left(\begin{array}{l}
E_{x} \\
E_{y} \\
E_{z}
\end{array}\right)=E_{o}\left(\begin{array}{c}
\frac{1}{\sqrt{2}} \sin \theta \\
-\frac{1}{\sqrt{2}} \sin \theta \\
\cos \theta \exp (i \Delta k d)
\end{array}\right)
$$

Equation (3.2) then defines the crystal frame polarization components

$$
\left(\begin{array}{c}
P_{x}^{(2)}(d) \\
P_{y}^{(2)}(d) \\
P_{z}^{(2)}
\end{array}\right)=2 \varepsilon_{o} E_{o}^{2}\left(\begin{array}{c}
-\sqrt{2} d_{14} \cos \theta \sin \theta \cos (\Delta k d) \\
\sqrt{2} d_{14} \cos \theta \sin \theta \cos (\Delta k d) \\
-d_{36} \sin ^{2} \theta
\end{array}\right)
$$

The oscillating $\cos (\Delta k d)$ dependence of $P_{x}^{(2)}(d)$ and $P_{y}^{(2)}(d)$, resulting from the birefriengence, leads to emission of periodically interfering $\mathrm{THz}$ field components which become increasingly negligible for crystal lengths greater than $2 \pi / \Delta k$. In contrast, the field components resulting from $P_{z}^{(2)}$ increase linearly with crystal length, assuming good phase matching of the optical and $\mathrm{THz}$ waves. In (110)-cut $\mathrm{ZnGeP}_{2}, 2 \pi / \Delta k \approx 30 \mu \mathrm{m}$ for $1300 \mathrm{~nm}$ excitation [56], which will be demonstrated as an optimally phase-matched pump wavelength in Chapter 4. Typically samples used for collinearly pumped optical rectification are at least an 
order of magnitude longer than $2 \pi / \Delta k$ when the phase-matching condition is well satisfied; therefore, for most practical cases it is justified to neglect $P_{x}^{(2)}$ and $P_{y}^{(2)}$ completely, making the contribution from $d_{14}$ in this plane effectively nonexistent. Applying the transformations (3.4) and (3.5) to obtain the parallel and perpendicular $\mathrm{THz}$ components respectively gives

$$
\begin{gathered}
E_{T H z}^{\|}(\theta) \propto P_{\|}^{(2)}=-2 \varepsilon_{o} E_{o}^{2} d_{36} \cos \theta \sin ^{2} \theta \\
E_{T H z}^{\perp}(\theta) \propto P_{\perp}^{(2)}=2 \varepsilon_{o} E_{o}^{2} d_{36} \sin ^{3} \theta
\end{gathered}
$$

Figure 3.2 (a) and (b) plot calculated $E_{T H z}^{\|}$and $E_{T H z}^{\perp}$ for (110)-cut zincblende and positive-uniaxial chalcopyrite structures as a function of the azimuthal angle (solid blue and dashed red respectively). The two dependencies are on a relative scale, with $\varepsilon_{o} E_{o}^{2} d_{36}$ divided out, allowing for comparison of the THz field magnitudes. The maximum $E_{T H z}^{\|}$component for chalcopyrite crystals is reduced by a factor or three in comparison to the zincblende structure, but the maximum $E_{T H z}^{\perp}$ component remains strong in both structures. The above model is valid for crystal lengths shorter than the temporal walk-off length between o- and e-waves.

Assuming equal nonlinearity, the ratio of the maximum effective second-order nonlinear coefficients (zincblende/chalcopyrite) is $2 / \sqrt{3} \approx 1.155$. Thus, the nonlinear coefficients of a zincblende crystal may be accessed more efficiently for optical rectification in the (110) plane than those of a chalcopyrite crystal. It will be shown in Section 3.3 that the (110) plane is, in

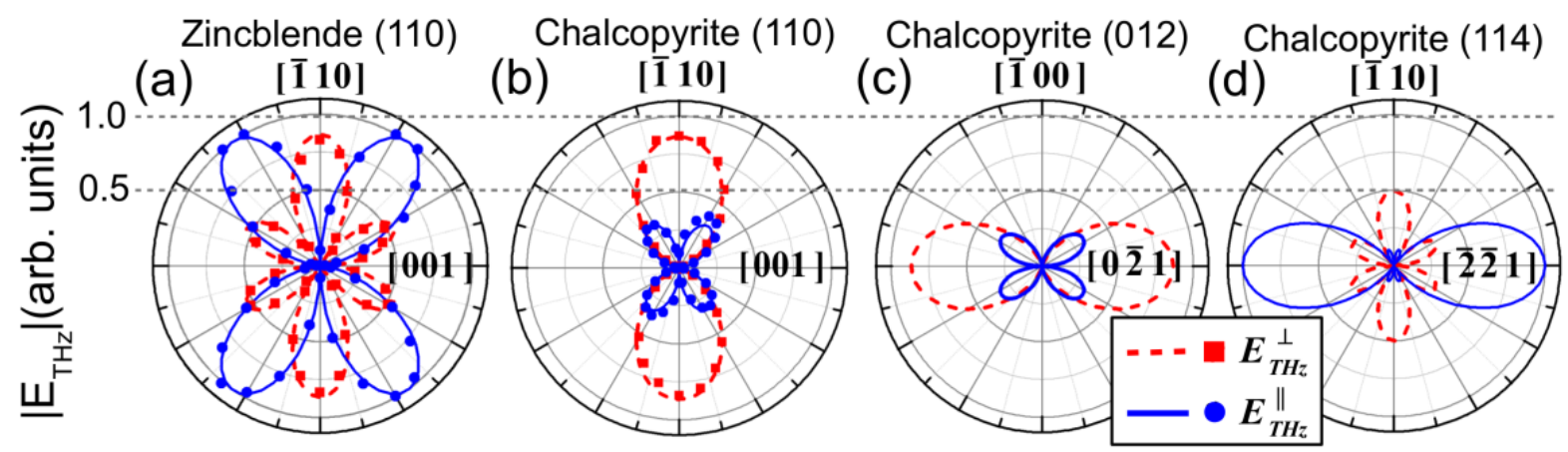

Figure 3.2: Experimental and theoretical THz peak-to-peak field amplitude for a (a) (110)-cut GaP crystal with zincblende structure and (b) (110)-cut $\mathrm{ZnGeP}_{2}$ crystal with chalcopyrite structure and uniaxial birefringence. Theoretical prediction of the THz emission for uniaxial chalcopyrite structures cut in the (c) (012) plane and (d) (114) plane. 
fact, not the most efficient plane of generation for chalcopyrite crystals: the above ratio defining how efficiently the crystal nonlinearity accessed may be equal to unity.

\subsection{Experimental Verification}

The angular dependence of $\mathrm{THz}$ emission was measured in as-grown $\mathrm{ZnGeP}_{2}$ and undoped GaP samples with thicknesses of $0.33 \mathrm{~mm}$ and $0.1 \mathrm{~mm}$ respectively. The orientation of this particular $\mathrm{ZnGeP}_{2}$ crystal was verified by electron paramagnetic resonance, ensuring that the $\langle 001\rangle$ direction is in the plane of the sample [110]. GaP is commercially available. Measurements were performed at normal pump incidence with the crystal rotated about the axis of the pump wavevector. Measurements employed $\sim 100$ fs pulses from a $1 \mathrm{kHz}$ regenerative amplifier and OPA. For GaP and $\mathrm{ZnGeP}_{2}$ the pump pulses were tuned to center wavelengths of $800 \mathrm{~nm}$ and $1300 \mathrm{~nm}$ respectively. EO sampling, with linearly polarized $800 \mathrm{~nm}$ probe pulses and a (110)-cut ZnTe crystal, was used to detect the THz field. As expected, the measured spectra were broadband ( $0.5 \mathrm{THz}$ to $3 \mathrm{THz}$ ) since the emission from $\mathrm{GaP}$ or $\mathrm{ZnGeP}_{2}$ is not limited by absorption due to IR-active phonons within this range. For $\mathrm{ZnGeP}_{2}$, absorption in this frequency range is known to be similar or smaller than that of $\mathrm{ZnTe}, \mathrm{GaP}$ and $\mathrm{LiNbO}_{3}$, but similar to that of GaAs [36]. As explained in Section 2.2.1, the ZnTe detector and probe pulse were oriented to detect a single THz polarization. To selectively acquire either $E_{T H z}^{\|}$or $E_{T H z}^{\perp}$, the detection crystal and probe pulse polarization are rotated by $90^{\circ}$. Field amplitudes are obtained by extracting the peak-to-peak voltage (see Figure 2.4 (b)) in individual THz transients as a function of $\theta$.

The resulting angle-dependent field amplitudes for (110)-cut GaP and $\mathrm{ZnGeP}_{2}$ are shown in Figure 3.2 (a) and (b) respectively. The data exhibit excellent agreement with the model described above. For the (110) plane in which the data and model are compared, because $\mathrm{ZnGeP}_{2}$ is significantly longer than $2 \pi / \Delta k$, the only surviving nonzero tensor element in the model is $d_{36}$; see Equations (3.12) and (3.13). Consequently, for this orientation, discussion of the differences between zincblende and chalcopyrite $\mathrm{THz}$ responses resulting from the nonlinear tensor is moot: birefringence alone is sufficient to describe the significant differences. 


\subsection{Predictions}

The possibility of efficient generation from other crystal orientations of $\mathrm{ZnGeP}_{2}$ are here explored using the analytical approach of Section 3.1.2. Due to lattice symmetry the chalcopyrite (012) plane (equivalent to the zincblende (011) plane) is expected to show similar behavior to that of the (110) plane. Also, in zincblende crystals the $\{112\}$ planes shows equal efficiency for optical rectification to the $\{110\}$ planes [111]; thus, the equivalent chalcopyrite (114) plane is also of interest. Predictive analysis is therefore performed for the (012) and (114) chalcopyrite planes. The only significant alteration to analysis for the (110) plane is the arbitrary form of the pump field in a given plane and the transformation back to the lab reference frame.

For the (012) plane the $x$-axis is in the plane and the $z$ and $y$ axes are fixed at $45^{\circ}$ to the plane. For the (114) plane no axis lies in the cut plane. For both planes the azimuthal angle $\theta$ is generalized as the angle between the projection of the z-axis onto the crystal cut plane and the incident linear polarization direction (confined to the cut plane). The pump fields are then expressed in the crystal frame as the sum of the o- and e-waves:

$$
\begin{gathered}
\vec{E}^{(012)}=E_{o}\left\{\left(\begin{array}{c}
-1 \\
0 \\
0
\end{array}\right) \sin \theta+\frac{1}{\sqrt{2}}\left(\begin{array}{c}
0 \\
-1 \\
1
\end{array}\right) \cos \theta \exp (i \Delta k d)\right\} ; \\
\vec{E}^{(114)}=E_{o}\left\{\frac{1}{\sqrt{2}}\left(\begin{array}{c}
-1 \\
1 \\
0
\end{array}\right) \sin \theta+\frac{1}{\sqrt{3}}\left(\begin{array}{c}
-1 \\
-1 \\
1
\end{array}\right) \cos \theta \exp (i \Delta k d)\right\} .
\end{gathered}
$$

The relative accumulated phase between 0 - and e-waves is assigned to the e-wave. The transformations back to the crystal frame for the (012) and (114) orientations are:

$$
P_{\|}^{(2),(012)}=\left\{\left(\begin{array}{lll}
-1 & 0 & 0
\end{array}\right) \sin \theta+\frac{1}{\sqrt{2}}\left(\begin{array}{lll}
0 & -1 & 1
\end{array}\right) \cos \theta\right\}\left(\begin{array}{l}
P_{x}^{(2)} \\
P_{y}^{(2)} \\
P_{z}^{(2)}
\end{array}\right)
$$




$$
\begin{aligned}
& P_{\perp}^{(2),(012)}=\left\{\left(\begin{array}{lll}
-1 & 0 & 0
\end{array}\right) \cos \theta+\frac{1}{\sqrt{2}}\left(\begin{array}{lll}
0 & -1 & 1
\end{array}\right) \sin \theta\right\}\left(\begin{array}{l}
P_{x}^{(2)} \\
P_{y}^{(2)} \\
P_{z}^{(2)}
\end{array}\right) ; \\
& P_{\|}^{(2),(114)}=\left\{\frac{1}{\sqrt{2}}\left(\begin{array}{lll}
-1 & 1 & 0
\end{array}\right) \sin \theta+\frac{1}{\sqrt{3}}\left(\begin{array}{lll}
-1 & -1 & 1
\end{array}\right) \cos \theta\right\}\left(\begin{array}{l}
P_{x}^{(2)} \\
P_{y}^{(2)} \\
P_{z}^{(2)}
\end{array}\right) \\
& P_{\perp}^{(2),(114)}=\left\{\frac{1}{\sqrt{2}}\left(\begin{array}{lll}
-1 & 1 & 0
\end{array}\right) \cos \theta+\frac{1}{\sqrt{3}}\left(\begin{array}{lll}
-1 & -1 & 1
\end{array}\right) \sin \theta\right\}\left(\begin{array}{l}
P_{x}^{(2)} \\
P_{y}^{(2)} \\
P_{z}^{(2)}
\end{array}\right)
\end{aligned}
$$

Application of these transformations give the predicted relative $\mathrm{THz}$ field amplitudes as a function of rotation angle for the measured parallel and perpendicular components emitted for normal pump incidence to the (012) and (114) planes:

$$
\begin{gathered}
E_{T H z}^{\|,(012)} \propto P_{\|}^{(2),(012)}=2 \varepsilon_{o} E_{o}^{2} d_{14} \cos ^{2} \theta \sin \theta ; \\
E_{T H z}^{\perp,(012)} \propto P_{\perp}^{(2),(012)}=2 \varepsilon_{o} E_{o}^{2} d_{14} \cos ^{3} \theta ; \\
E_{T H z}^{\|,(114)} \propto P_{\|}^{(2),(114)}=\frac{1}{3 \sqrt{3}} \varepsilon_{o} E_{o}^{2} \cos \theta\left[8 d_{14} \cos ^{2} \theta+d_{36}(-1+5 \cos 2 \theta)\right] ; \\
E_{T H z}^{\perp,(114)} \propto P_{\perp}^{(2),(114)}=\frac{1}{3 \sqrt{3}} \varepsilon_{o} E_{o}^{2} \sin \theta\left[8 d_{14} \cos ^{2} \theta+d_{36}(-1+5 \cos 2 \theta)\right] .
\end{gathered}
$$

Based on these results, Figure 3.2 (c) and (d) show predictions of the angle-dependent $\mathrm{THz}$ generation for uniaxial birefringent chalcopyrite crystals cut in the (012) and (114) planes respectively. Each of these planes has a different near-infrared extraordinary index of refraction, but neither value varies as a function of $\theta$. Each crystal cut then exhibits unique phase matched pump wavelengths as will be discussed in the following chapter.

To understand the results it helps to inspect the crystal structures and orientations; see Figure 3.3. For zincblende structures in the (110) plane the bonds show an angle $\pm 54.7^{\circ}$ away from the [001] projection. This is the angle at which $E_{T H z}^{\|}$is maximized; see Figure 3.2 (a). For $\mathrm{ZnGeP}_{2}$ the bond angle is similar, if the noncubic structure is ignored, but the birefringence 


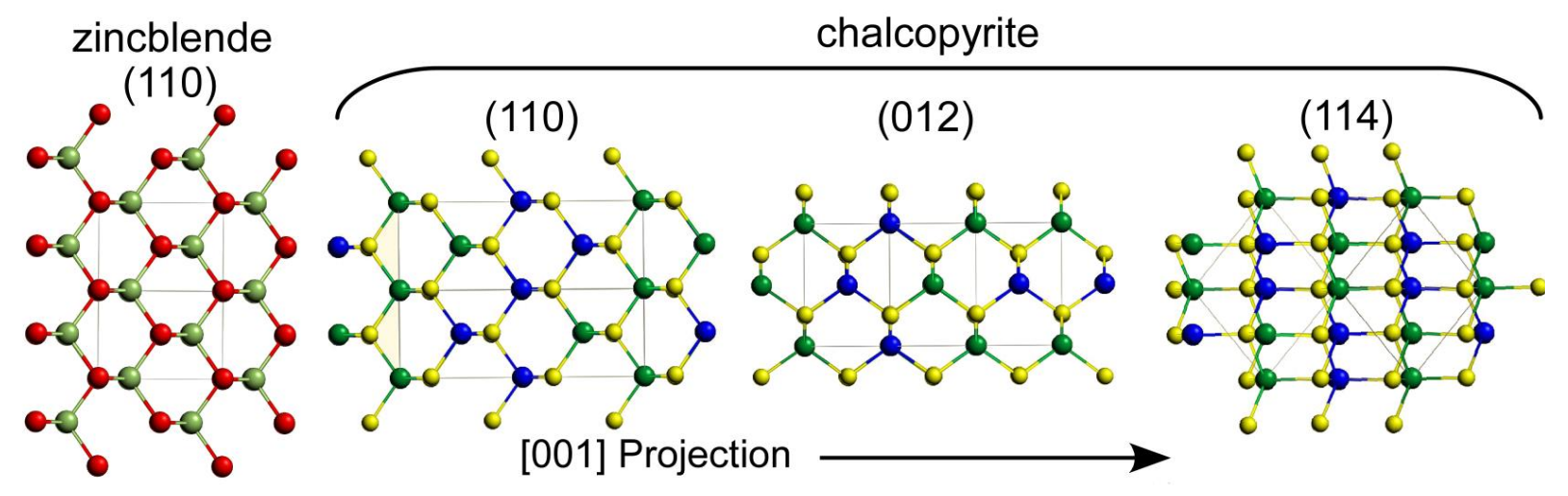

Figure 3.3: Zincblende and chalcopyrite crystal structures for the planes considered. The arrow shows the direction of the [001] projected onto the plane of the page.

strongly suppresses the $\mathrm{THz}$ generation for pump fields oriented at that angle. Reorienting the chalcopyrite to the (012) plane effectively rotates the bonds and the $\mathrm{THz}$ response by $90^{\circ}$ relative to the [001] projection, as seen by comparing Figure 3.2 (b) and (c). At the rotation angle for maximum efficiency in each of these planes phase matching is best achieved for perpendicular $\mathrm{THz}$ and pump polarizations, i.e. ooe in the (110) crystal plane and eeo in the (012) plane. These configurations will be referred to as (110)-ooe and (012)-eeo in this and later chapters. In this notation the first two waves correspond to the pump pulse and the third corresponds to the emitted $\mathrm{THz}$ pulse. Each orientation exhibits a unique phase matching condition. This can be considered as a relaxation of the singular phase matching condition observed in zincblende crystals.

For chalcopyrite crystals, the analysis shows other efficient configurations. In fact, stronger emission should be observed for (114)-cut crystals. All of the angle dependences in Figure 3.2 are plotted on the same relative scale, considering only the effects of birefringence with identical physical parameters (e.g. nonlinear tensor elements). For pumping normal to the (114) plane, recovery of the maximum zincblende effective nonlinear coefficient (assuming $\left.d_{36}=d_{14}\right)$ is predicted; compare the maximum amplitudes of Figure 3.2 (a) and (d) for $E_{T H z}^{\|}$. For this orientation the crystal is rotated such that the bonds discussed above are now parallel to the [001] projection onto the crystal surface. That is, the pump pulse accesses the most efficient polarization direction but avoids the effects of birefringence. In the (114) plane the 


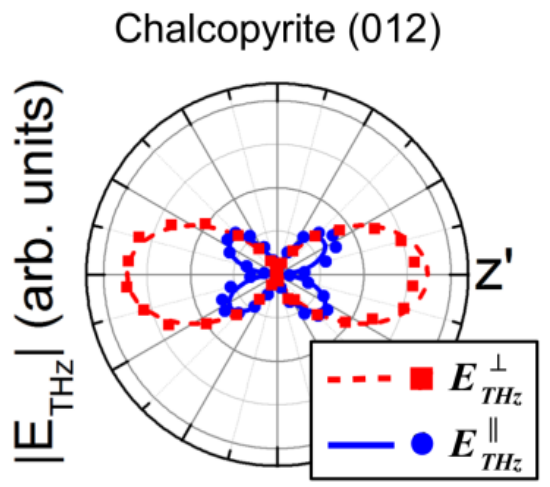

Figure 3.4: Experimental verification of the modeled THz emission as a function of rotation angle. $0^{\circ}$ represents the pump polarization along the $z^{\prime}$-axis.

most efficient phase-matching condition is eee, where all fields are collinearly polarized along the [001] projection.

Since the above work was published [112] a (012) cut crystal was generously provided by BAE Systems. The rotation dependence in the (012) plane has been experimentally verified with the data and model shown in Figure 3.4. As for the (110) plane good agreement is observed between and data and model.

\subsection{Chapter Summary}

As has been discussed, chalcopyrite crystals in the form of $\mathrm{ZnGeP}_{2}$ are promising and useful materials for $\mathrm{THz}$ generation by near-infrared laser pulses. The experimental and modeled results performed here disentangle the effects of birefringence on the orientation dependence of $\mathrm{THz}$ emission efficiency from the fundamental orientation dependence on the nonlinear susceptibility; birefringence modifies the angular dependence of $\mathrm{THz}$ emission from ternary chalcopyrite structured semiconductors in comparison to their zincblende binary analogs. Birefringence is shown to reduce the maximum effective nonlinear coefficient for the commonly used (110) crystal orientation, which is often chosen based solely on the secondorder tensor. The above calculations predict that generation with the pump polarization rotated for maximum efficiency in the (114) plane recovers the maximum effective nonlinear 
coefficient: the most efficient rotation angle in this plane is not affected by birefringence. The work summarized in this chapter highlights how birefringence plays a role in relaxing the phasematching condition for $\mathrm{THz}$ generation in efficient emitters, without significant detrition in the efficiency. The model approach can easily be extended to other birefringent materials, given the appropriate second-order nonlinear optical susceptibility tensor. 


\section{Chapter 4 Phase Matching in Chalcopyrite Structured Crystals}

In Section 2.1.1.2 the importance of phase-matched generation for efficient and broadband $\mathrm{THz}$ optical rectification was discussed. The coherence length was defined and calculated maps were presented for $\mathrm{THz}$ optical rectification in zincblende semiconductors of current interest. In this chapter (Section 4.1), the coherence length is calculated and mapped as a function of pump wavelength and $\mathrm{THz}$ frequency for a number of chalcopyrite crystals. The crystals examined represent the few chalcopyrite materials for which electro-optic properties and dispersion relations are available at near-infrared and $\mathrm{THz}$ frequencies. Other parameters relevant to $\mathrm{THz}$ optical rectification in these chalcopyrites are tabulated and discussed. In Section 4.2, the distinct phase-matching conditions for generation in the chalcopyrite (110) and (012) planes (see Chapter 3) are experimentally verified in $\mathrm{ZnGeP}_{2}$ by observing generation efficiency as a function of pump wavelength. A deeper analysis of the data provides an experimental mapping of a $\mathrm{THz}$ optical-rectification waveform over a broad phase-mismatch (i.e. pump wavelength) range in the time and frequency domains. The frequency-dependent terahertz waveform is plotted against the pump wavelength to show a full map of the generation response. Since these maps take into account phase matching, among other properties affecting the generation, we refer to them as "phase-matching dispersion maps."

\subsection{Chalcopyrite Coherence Length Mapping}

Prior to the work of this dissertation, chalcopyrite structured semiconductors were unexplored as pulsed broadband $\mathrm{THz}$ sources, likely due to the scant number of high quality crystals that are commercially available. In addition, the linear and nonlinear optical properties of chalcopyrites relevant to $\mathrm{THz}$ optical rectification are only available for a small number of the numerous ternary semiconductors. To make this information readily accessible and to present a first general look at ternary semiconductors as THz emitters, it is worthwhile to discuss crystals 
for which parameters defining optical-rectification phase matching (near-infrared and $\mathrm{THz}$ frequency dispersion relations) and efficiency (EO coefficients) have been experimentally defined. These crystals include the group I-III- $-\mathrm{VI}_{2}$ semiconductors $\mathrm{AgGaSe} \mathrm{Ag}_{2} \mathrm{AgGaS}$, and $\mathrm{CuGaS}_{2}[56,113-115]$ as well as group II-IV-V $\mathrm{V}_{2}$ semiconductors $Z n G_{e P}, \mathrm{CdGeP}_{2}$, and $\mathrm{CdGeAs}_{2}[56,83,97,116-118]$. For these materials the complex dielectric constants were determined in the $\mathrm{THz}$ range by fitting reflectivity/transmission data with Lorentz oscillator models. Using these frequency dependent dielectric constants and near-infrared dispersion relations, the coherence length for the above chalcopyrite semiconductors is mapped. The potential of these chalcopyrite semiconductors for broadband and efficient $\mathrm{THz}$ optical rectification is discussed in the context of these calculations and other relevant parameters; see Table 1. The calculated coherence length maps for GaAs, GaP, and ZnTe were presented in Figure 2.2 and are useful for comparison to the chalcopyrite maps. The Lorentz oscillator models fit the strength and frequency of far-infrared phonon resonances; the effects of these phonon resonances are clearly apparent in the coherence-length maps presented.

Some parameters relevant to $\mathrm{THz}$ optical rectification for the above chalcopyrite semiconductors are presented in Table 1. Parameters for zincblende semiconductors of interest are also given for reference. References for the parameters presented in Table 1 are: (1) [119]; (2) [56]; (3) [120]; (4) [121]; (5) [36]; (6) [122]; (7) [123]; (8) [124]. Recall from section 2.1.1 that the EO coefficient and near-infrared refractive index primarily determine the relative efficiency of THz emission by optical rectification: $\chi_{i j k}^{(2)}=-\left(n^{4} / 2\right) r_{i j k}$ [79]. The nonlinear coefficients $\left(d_{m n}=\chi_{i j k}^{(2)} / 2\right)$ in Table 1 are calculated using this relation and the near-infrared refractive index $\left(n_{o}\right)$ at phase-matched pump wavelengths specified in the final column. The band-gap is provided for easy determination of the onset of 2PA and 3PA at photon energies at $1 / 2$ and $1 / 3$ of the band-gap respectively; see Section 2.1.1.3. For all crystals phase-matched pump wavelengths fall well within the near-infrared transparency range with the exceptions of $\mathrm{CdGeAs}_{2}$ and $\mathrm{AgGaS}_{2}$; these crystals show phase matching near the single-photon absorption edge. 
Table 1: Parameters for the chalcopyrite semiconductors with coherence length mapped in Figure 4.1 and Figure 4.3. In the final column the phase-matched pump wavelengths are given for the (110)-ooe and (012)-eeo configurations. Where not referenced, the nonlinear coefficients are calculated using the refractive index at the phase-matched wavelength specified in the last column for o-wave pump (left).

\begin{tabular}{|c|c|c|c|c|c|}
\hline Material & $\begin{array}{c}\text { Transparency } \\
\text { Range } \\
(\mu \mathrm{m})\end{array}$ & $\begin{array}{c}\text { Electro-Optic } \\
\text { Coefficient } \\
\text { (pm/V) }\end{array}$ & $\begin{array}{l}\text { Nonlinear } \\
\text { Coefficient } \\
\text { (pm/V) }\end{array}$ & $\begin{array}{c}\text { Band-Gap } \\
(\mathrm{eV})\end{array}$ & $\begin{array}{c}\text { Phase-Matched (1 THz) } \\
\text { [(110)-ooe/(012)-eeo] } \\
(\mu \mathrm{m})\end{array}$ \\
\hline $\mathrm{ZnGeP}_{2}$ & $0.74-15^{1}$ & $r_{41}=1.6^{4}$ & $d_{14}=39.4^{5}$ & $2.14^{8}$ & $1.16 / 1.28$ \\
\hline $\mathrm{CdGeP}_{2}$ & $0.7-14^{3}$ & $r_{41}=3.0^{3}$ & $\mathrm{~d}_{14}=87.9$ & $1.73^{8}$ & $1.27 / 1.44$ \\
\hline $\mathrm{CdGeAs}_{2}$ & $2.2-18^{2}$ & ----- & $\begin{array}{l}---- \\
--\end{array}$ & $0.57^{8}$ & $-----/ 2.2$ \\
\hline $\mathrm{CuGaS}_{2}$ & $0.5-13^{2}$ & $r_{41}=1.9^{4}$ & $\mathrm{~d}_{14}=20.7$ & $2.43-2.55^{8}$ & $0.88 / 0.70$ \\
\hline $\mathrm{AgGaSe}_{2}$ & $0.78-18^{1}$ & $r_{41}=4.5^{7}$ & $\mathrm{~d}_{14}=68.2$ & $1.8^{8}$ & $0.79 / 0.94$ \\
\hline $\mathrm{AgGaS}_{2}$ & $0.61-13.5^{1}$ & $r_{41}=4.0^{6}$ & $\mathrm{~d}_{14}=83.2$ & $2.7^{8}$ & $0.60 / 0.54$ \\
\hline GaAs & $0.9-17.3^{1}$ & $r_{41}=1.5^{5}$ & $d_{14}=46.1^{5}$ & $1.42^{8}$ & 1.40 \\
\hline GaP & $0.54-10.5^{1}$ & $r_{41}=0.94^{5}$ & $\mathrm{~d}_{14}=21.7^{5}$ & $2.27^{8}$ & 1.03 \\
\hline ZnTe & $0.55-25^{1}$ & $r_{41}=4.0^{5}$ & $d_{14}=66^{5}$ & $2.35^{8}$ & 0.84 \\
\hline
\end{tabular}

Coherence length maps for the II-IV $-\mathrm{V}_{2}\left(\mathrm{I}-\mathrm{III}-\mathrm{VI}_{2}\right)$ chalcopyrites are shown in Figure 4.2 (Figure 4.3). These calculations assume a linear pump polarization confined to the efficient (110) and (012) planes and rotated within the planes for maximum efficiency. For pump polarization confined to the (110) plane the most efficient configuration is ooe and for the (012) plane the most efficient configuration is eeo; see Section 3.3. Phase-matching conditions for the efficient (012)-eeo and most efficient (114)-eee configurations are similar, so a coherence length map for the latter is not shown. Because the crystal z-axis lies in the (110) plane the angle between the pump wavevector and the z-axis is $\phi=90^{\circ}$ and $n_{e}\left(\nu_{T H z}, \phi\right)=n_{e}\left(\nu_{T H z}\right)$; that is, the principle extraordinary index $n_{e}\left(\nu_{T H z}\right)$ need only be considered in calculating the coherence length in this plane. For the (114) plane the pump wavevector forms an angle of $\phi=45^{\circ}$ with the z-axis at normal incidence and the near-infrared extraordinary index $n_{e}\left(\nu_{N I R}, \phi\right)$, is defined by the relation [74]: 


$$
\frac{1}{n_{e}\left(v_{N I R}, \phi\right)^{2}}=\frac{\sin ^{2} \phi}{n_{e}\left(v_{N I R}\right)^{2}}+\frac{\cos ^{2} \phi}{n_{o}\left(v_{N I R}\right)^{2}} .
$$

Calculated maps for all chalcopyrite crystals clearly show unique phase-matching conditions in the (110)-ooe and (012)-eeo configurations in contrast to the singular condition in isotropic zincblend semiconductors. This is emphasized in the last column of Table 1 where optimal phase-matched pump wavelengths are listed for thick crystals in the (110)-ooe and (012)-eeo configurations at $1 \mathrm{THz}$. A general characteristic evident from these maps (and the zincblende maps) is that the optimal phase-matched pump wavelength for thick crystals (e.g. L $>10 \mathrm{~mm}$ ) is not the optimal pump wavelength for broadest generation bandwidth in thinner crystals. For example, in (110) $\mathrm{ZnGeP}_{2}$ the optimal pump wavelength is $\sim 1.16 \mu \mathrm{m}(1.07 \mathrm{eV})$ for thick crystals, but for a $150 \mu \mathrm{m}$ thick crystal pumping near $0.85 \mu \mathrm{m}(1.46 \mathrm{eV})$ will give the maximum potential generation bandwidth.

Disruption in the continuity of phase matching at lower $\mathrm{THz}$ frequencies is observed in most chalcopyrite maps (not present in zincblende semiconductors), due to weak lowfrequency phonon resonances. These resonances will also lead to absorption lines in the generated $\mathrm{THz}$ bandwidth; the strength and bandwidth of the absorption will depend on the phonon oscillator strength and damping. These weak low-frequency phonons represent splitting of the zincblende phonon modes and are attributable to differences in force constants for the II-V and IV-V bonds [125]. Depending on the resonance strength, dispersion measurement method, and fitting model, the effects of some weak low-frequency phonon resonances may not appear in the $\mathrm{THz}$ dispersion relations applied in calculating coherence lengths.

GaP shows particular promise among zincblende semiconductors for broad bandwidth generation and detection, as is clear from comparison of Figure 2.2 (a)-(c). This was directly demonstrated by $\mathrm{Wu}$ et al. using $150 \mu \mathrm{m}$ thick crystals for both generation by optical rectification and EO detection [86]; seven $\mathrm{THz}$ of detection bandwidth was achieved. Comparatively, (110) oriented $\mathrm{ZnGeP}_{2}$ and $\mathrm{CdGeP}_{2}$ show similar potential for broadband generation and detection; see Figure 1.2 (a) and (c). The effect of a weak phonon resonance at $\sim 3.5 \mathrm{THz}$ in the map for (110)-ooe oriented $\mathrm{ZnGeP}_{2}$ is apparent, but causes minimal phase 

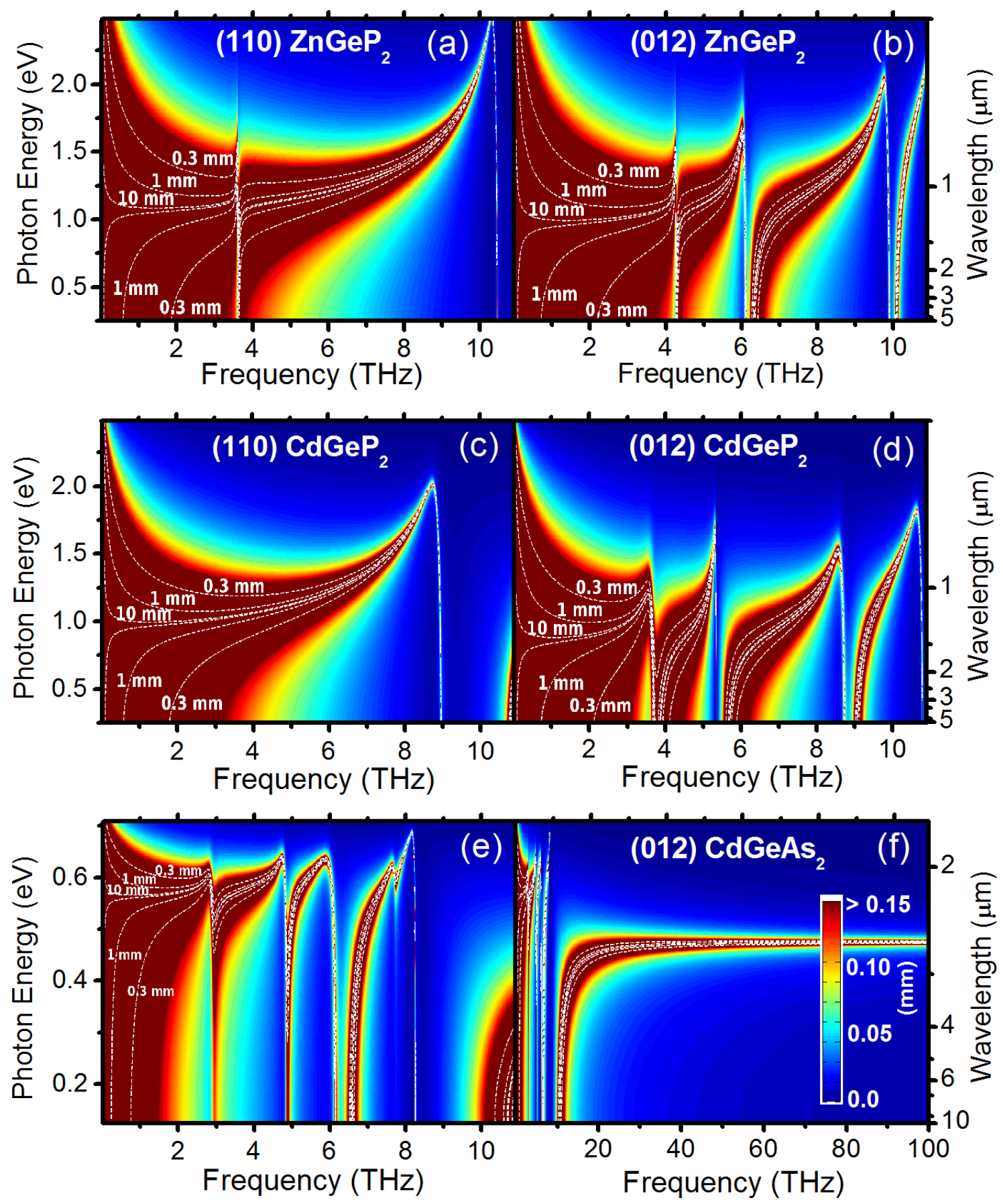

Figure 4.1: Coherence length calculated from near-infrared and THz dielectric dispersion relations for $\mathrm{ZnGeP}_{2}$ with (a) o-wave pump and e-wave THz in the (110) plane, (b) e-wave pump and o-wave THz in the (012) plane. (c) and (d): Calculated coherence length for $\mathrm{CdGeP}_{2}$ with the same orientation as in (a) and (b) respectively. (e) and (f): Calculated coherence length for $\mathrm{CdGeAs}_{2}$ with e-wave pump and o-wave THz in the (012) plane. (f) is scaled to show the distinctive broad band of phase matching beyond $10 \mathrm{THz}$. For all maps, the color scale saturates at $150 \mu \mathrm{m}$, and two appropriately labeled dashed lines confine regions with coherence length greater than $0.3 \mathrm{~mm}, 1.0 \mathrm{~mm}$, and $10 \mathrm{~mm}$. The color scale for all plots is shown in (f).

mismatch. The corresponding resonance in $\mathrm{CdGeP}_{2}$ was too weak to be observed in a thorough 
study of its phonon vibrational properties [126]. Phase-matched pump pulses for $\mathrm{ZnGeP}_{2}$ and $\mathrm{CdGeP}_{2}$ in the (110)-ooe orientations are shifted to lower photon energies in comparison to GaP; the (012)-eeo orientations show broadband phase matching at even lower photon energies. The stronger low-frequency phonon resonances for the latter orientation lead to considerable disruption of phase matching beyond about $\sim 4 \mathrm{THz}$ in both crystals. As a result of their long-wavelength pump phase matching, both crystals show promise for near-infrared generation with fiber lasers. In particular, (012) $\mathrm{ZnGeP}_{2}$ Shows good phase matching for Cr:forsterite lasers and high-energy laser amplifiers ( $1.24 \mu \mathrm{m}) ;(012) \mathrm{CdGeP}_{2}$ shows particular promise among semiconductors generally for generation using compact and cost-effective 1.55 $\mu \mathrm{m}$ fiber lasers.

Due to sufficiently wide band-gaps, broadband phase matching is achievable in $\mathrm{ZnGeP}_{2}$ and $\mathrm{CdGeP}_{2}$ below the onset of $2 \mathrm{PA}$, as in $\mathrm{GaP}$. This is an important feature for efficient generation at high pump intensities; see Section 2.1.1.3. Figure 4.2 explicitly charts phase matched wavelengths (at $1 \mathrm{THz}$ ) and the range of strong $2 \mathrm{PA}$ in $\mathrm{ZnGeP}$ and $\mathrm{CdGeP}_{2}$ in comparison to the same in GaP and GaAs. In the latter materials long-wavelength pumping has already been explored to avoid the negative effects of 2PA [37-39]. For GaAs, with the use of
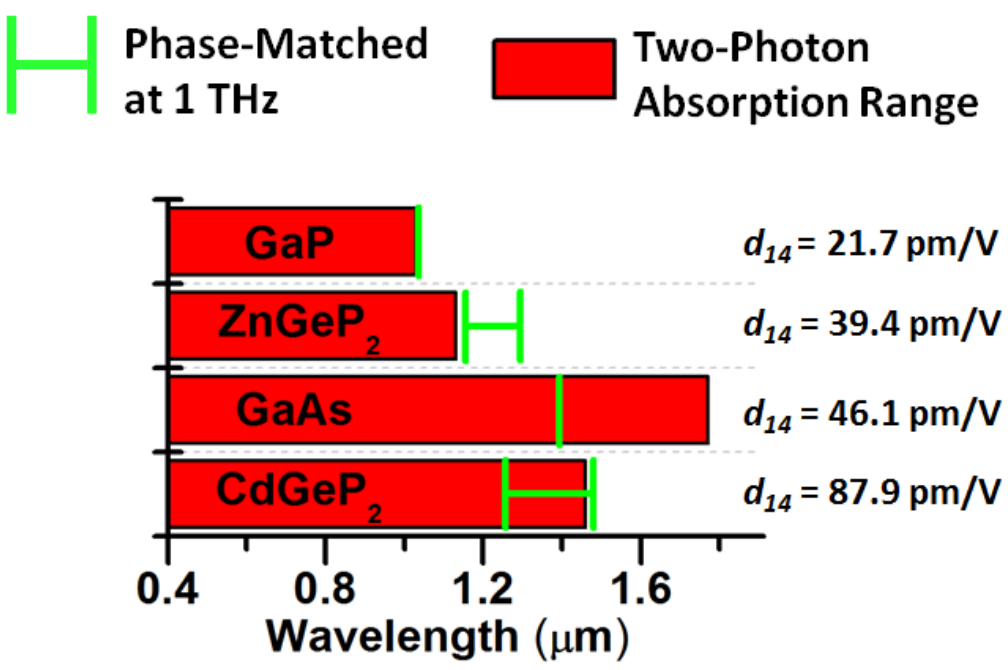

Figure 4.2: Regions of significant two-photon absorption and phase-matched wavelengths (at $1 \mathrm{THz}$ ) for THz optical rectification in some zincblende and chalcopyrite semiconductors. The crystals are listed in order of second order nonlinear coefficient magnitude. For the ternary crystals vertical green lines represent tunable phase matching in the (110)-ooe and (012)-eeo configurations. Wavelengths/photon energies between these orientations may be phase matched by angle tuning with variable efficiency. 
thin crystals, significant bandwidth of generation and detection is achievable below the onset of 2PA absorption near $1.75 \mu \mathrm{m}(0.708 \mathrm{eV})$ as seen in Figure 2.2 (c). While $\mathrm{CdGeP}_{2}$ shows similar phase matching to that of $\mathrm{GaAs}$, the nonlinear coefficient of $\mathrm{CdGeP}_{2}$ is almost twice that of GaAs. $\mathrm{CdGeP}_{2}$ also has a significantly lower onset for $2 \mathrm{PA}$. In fact, $\mathrm{CdGeP}_{2}$ appears to be the only semiconductor showing broadband phase matching while avoiding 2PA at $1.55 \mu \mathrm{m}$. Although high-quality growth of $\mathrm{CdGeP}_{2}$ has been achieved [127] and this material is of interest for potential spintronics applications [128,129], this crystal has not become commercially available because it lacks sufficient birefringence for phase-matched near-infrared nonlinear frequency conversion. However, the calculations and parameters presented here suggest that $\mathrm{THz}$ optical rectification in $\mathrm{CdGeP}_{2}$ shows excellent potential for applications requiring efficient and broadband $\mathrm{THz}$ emission. Commercially available $\mathrm{ZnGeP}_{2}$ exhibits a large nonlinear coefficient and shows no $2 \mathrm{PA}$ at phase-matched wavelengths. At a minimum, $\mathrm{ZnGeP}_{2}$ and $\mathrm{CdGeP}_{2}$ fill a significant collinear phase-matching gap between $\mathrm{GaP}$ and $\mathrm{GaAs}$ for $\mathrm{THz}$ optical rectification at long near-infrared wavelengths.

The coherence length of $\mathrm{CdGeAs}_{2}$ is mapped for the (012)-ooe configuration in Figure 4.1 (e) and (f). For the (110)-ooe configuration (not shown) phase-matched pump photon energies are well within the single-photon absorption range. While EO coefficients have not been measured for $\mathrm{CdGeAs}_{2}$, this crystal is of interest due to its considerably large nonlinearity. However, in the 1-10 THz range, only a narrow ( 2-3 THz) bandwidth of phase-matched generation is possible, even for thin crystals. This crystal does show broadband phase matching above $10 \mathrm{THz}$; see Figure 4.1 (f). This phase-matched band could be exploited for generation beyond $15 \mathrm{THz}$ for pump pulses with sufficiently short durations ( $<25 \mathrm{fs})$.

The coherence lengths of the I-III- $\mathrm{VI}_{2}$ semiconductors $\mathrm{CuGaS}_{2}, \mathrm{AgGaSe}$, and $\mathrm{AgGaS} 2$ are shown in Figure 4.3 (a)-(f). None of these crystals show broadband phase matching below the onset of 2PA (for thick crystals). (110)-ooe oriented $\mathrm{CuGaS}_{2}$ (Figure 4.3 (a)) does show a significant phase-matched bandwidth for thin crystals below the onset of 2PA near $1.0 \mu \mathrm{m}$ (1.24 eV), but exhibits no significant advantage over GaP in terms of its nonlinear coefficient (efficiency). The calculation does show that $\mathrm{CuGaS}_{2}$ is phase matched within a standard Ti:sapphire laser tuning range $(\sim 0.7-1.0 \mu \mathrm{m})$, for thick crystals $(\sim 1.0 \mathrm{~mm})$, with significantly 

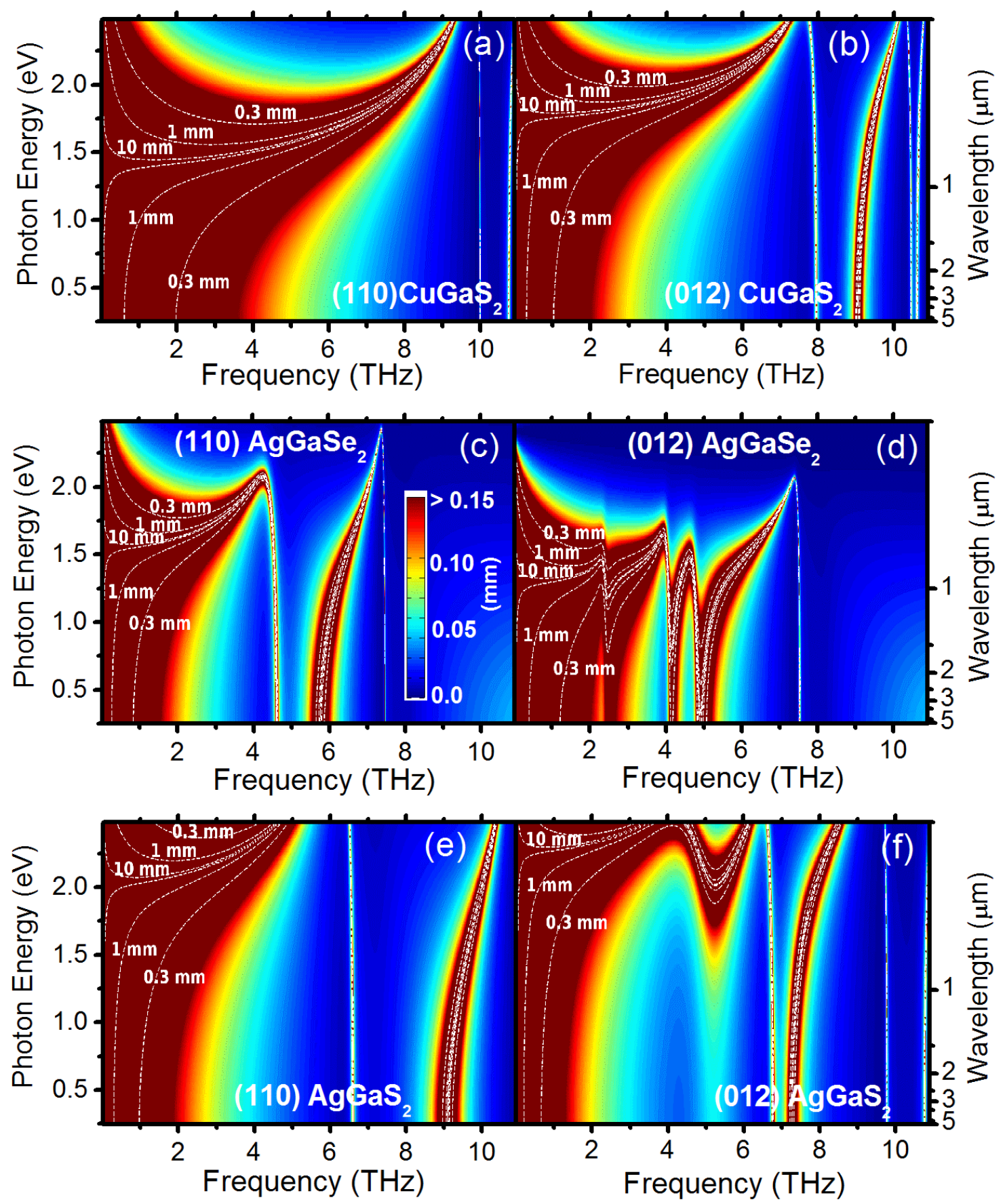

Figure 4.3: Coherence length calculated from near-infrared and $\mathrm{THz}$ dielectric dispersion relations for $\mathrm{CuGaS}_{2}$ with (a) o-wave pump and e-wave THz in the (110) plane, (b) e-wave pump and o-wave THz in the (012) plane. (c) and (d): Calculated coherence length for $\mathrm{AgGaSe}_{2}$ with the same orientation as in (a) and (b) respectively. (e) and (f): Calculated coherence length for $\mathrm{AgGaS}_{2}$ with with the same orientation as in (a) and (b) respectively. For all maps, the color scale saturates at $\mathbf{1 5 0} \mu \mathrm{m}$, and two appropriately labeled dashed lines confine regions with coherence length greater than $0.3 \mathrm{~mm}, 1.0 \mathrm{~mm}$, and $10 \mathrm{~mm}$. The color scale for all plots is shown in (c).

broader THz bandwidth than ZnTe; see Figure 2.2 (a). AgGaSe 2 (Figure 4.3 (c) and (d)) also 
exhibits phase matching within a standard Ti:sapphire laser tuning range and possesses nonlinear coefficients similar to those of ZnTe, but phase matching can only be achieved over a 3-4 $\mathrm{THz}$ bandwidth for thin crystals; thus, the only advantage $\mathrm{AgGaSe}_{2}$ might provide above $\mathrm{ZnTe}$ is some angle tuning of phase mismatch. $\mathrm{AgGaS}_{2}$ also possesses large nonlinearity, but is phase matched in the visible range. This crystal could fill a niche for $\mathrm{THz}$ pulse generation using femtosecond fiber lasers in the visible near $515 \mathrm{~nm}$. Both, $\mathrm{AgGaSe}_{2}$ and $\mathrm{AgGaS}_{2}$ are commercially available due to their applications in near-infrared frequency conversion.

\subsection{THz Waveform \& Phase Matching}

Here data are presented verifying phase-matched near-infrared wavelengths and $\mathrm{THz}$ frequencies for $\mathrm{ZnGeP}_{2}$ pumped in the (110)-ooe and (012)-eeo configurations (see Section 3.3). The data also give information concerning variation of efficiency with phase mismatch for collinear $\mathrm{THz}$ optical rectification. In Subsections 4.2.1 and 4.2.2, a deeper analysis of the data provides experimental time- and frequency-domain phase-matching dispersion maps of the generated $\mathrm{THz}$ waveform. (110)- and (012)-cut crystals of substantial thickness (3 mm) were used, making the effects of phase mismatch easily observable for wavelengths within the OPA tuning range. For data collection the crystal angles and time delays of the OPA were manually set, optimized, and tabulated for automatic recall at $20 \mathrm{~nm}$ intervals across the tuning range of the "signal" (1.12-1.62 $\mu \mathrm{m})$ and "idler" (1.625-2.5 $\mu \mathrm{m})$ pulses. Each wavelength was verified and optimized by observing the second-harmonic spectrum (generated in a BBO crystal) with a spectrometer spanning the visible range. Pump-beam alignment was accurately maintained with tuning across the signal wavelengths as verified well downstream of the $\mathrm{THz}$ source. A variable neutral-density filter and power meter were used to maintain the external pump power at $4 \mathrm{~mW}$ across the tuning range. Low intensity pumping was chosen to avoid any effects arising from nonlinear absorption and pulse reshaping in either the pump or $\mathrm{THz}$ beams.

Figure 4.4 shows the relative peak-to-peak THz field amplitude (see Figure 2.4 (b)) measured at each pump photon energy/wavelength. The peaks in efficiency for the (110)-ooe and (012)-eeo orientations agree well with the wavelengths calculated and recorded in the last 


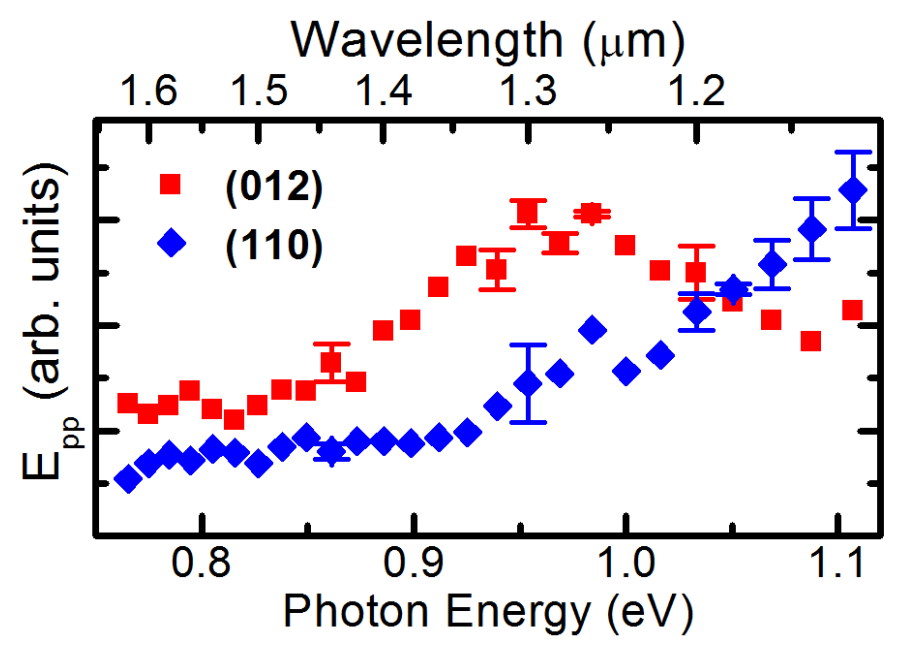

Figure 4.4: Peak-to-peak THz field amplitude of emission from $\mathrm{ZnGeP}_{2}$ in the (012)-eeo and (110)-ooe configurations as a function of central pump pulse photon energy/wavelength. The peaks in THz emission efficiency demonstrate optimized phase matching for each orientation. Error bars representing the standard deviation of multiple measurements include error associated with intrinsic laser source and detection noise plus any error in maintaining the pump power at $4 \mathrm{~mW}$.

column of Table 1. For both orientations the efficiency of generation drops to approximately one-third maximum far from optimal phase-matched pump wavelengths. Within error the two peaks in efficiency show the same peak-to-peak electric-field amplitude. As demonstrated by the analysis of Chapter 3, the nonlinear coefficients determining the relative efficiency for optical rectification in the (110) and (012) planes are $d_{36}$ and $d_{14}$. The data do not then indicate any considerable difference between the amplitudes of these coefficients.

\subsubsection{Time-Domain Mapping}

Each data point of Figure 4.4 represents the peak-to-peak of a full time-domain $\mathrm{THz}$ trace; see Figure 2.4 (b). A more complete picture of interference effects, extant for significant phase mismatch, on the emitted $\mathrm{THz}$ pulses can be gained by mapping the time-domain electric field amplitudes as a function of pump-photon energy. This mapping is shown for the (110)-ooe and (012)-eeo pump/emission configurations in Figure 4.5 (a) and (b) respectively; part (c) shows the (110) data from an alternate angle to better display the waveform profile. The timedomain data, collected at $20 \mathrm{~nm}$ pump intervals, is interpolated to produce continuous maps. The displayed pump range is extended beyond the OPA signal wavelengths of Figure 4.4 to the 

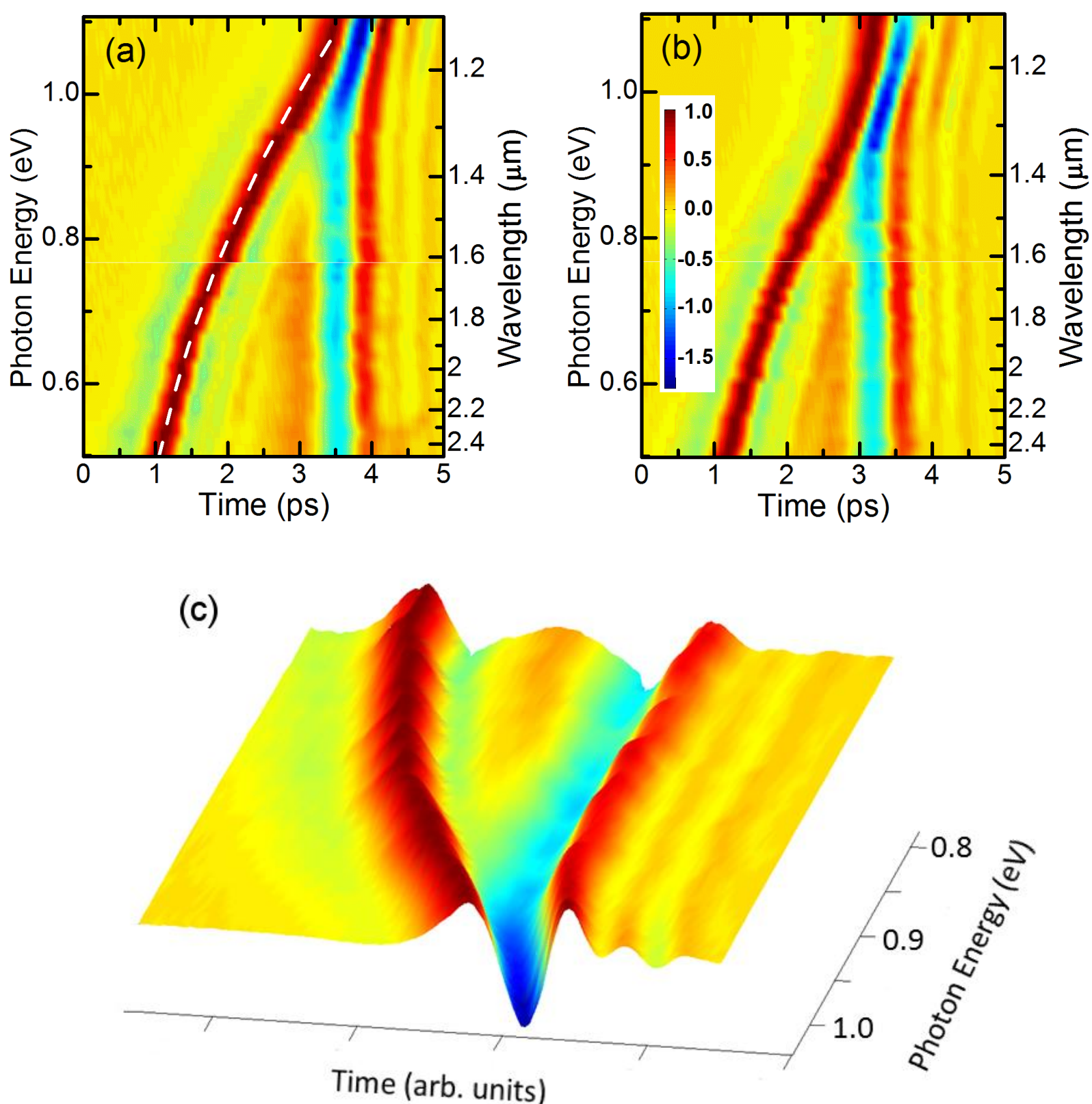

Figure 4.5: THz time-domain traces from $3 \mathrm{~mm}$ thick (a) (110)-cut and (b) (012)-cut $\mathrm{ZnGeP}$ source as a function of central pump photon energy/wavelength, normalized to the strongest leading peak (dark red). For good phase-matching near $1.1 \mathrm{eV}$ (a) and $0.97 \mathrm{eV}$ (b) single THz transients are observed. As the phase mismatch increases the emitted $\mathrm{THz}$ pulse splits into two distinct pulses. The dotted white line is a calculation of a relative time delay associated with the group velocity as a function of pump photon energy/wavelength. (c) shows the (110) data from an alternate angle.

idler tuning range to better resolve the effects of phase mismatch. Any potential effects from water absorption on the measured waveforms and spectra were eliminated by applying a nitrogen purge to the $\mathrm{THz}$ path from emitter to EO detection crystal. Each $\mathrm{THz}$ trace is 
arbitrarily normalized to the strongest leading peak amplitude (dark red) to emphasize the evolution of the $\mathrm{THz}$ waveform with pump wavelength (phase mismatch).

For good phase matching near $1.05 \mathrm{eV}(0.97 \mathrm{eV})$ in the (110)-ooe ((012)-eeo) configuration the $\mathrm{THz}$ traces exhibit a near single cycle waveform similar to those shown in Figure 2.4 (b) or Figure 2.7 (a). At lower pump energies, and increasing phase mismatch, division of the THz pulse into two distinct transients is observed. This splitting is consistent with models describing $\mathrm{THz}$ optical rectification with variable phase mismatch $[25,130,131]$ and experimental results at isolated pump wavelengths exhibiting phase mismatch $[25,132]$. For large phase mismatch, the models predict little contribution from the bulk of the nonlinear generation crystal due to interference; however, at the front and back crystal faces the interference is not complete. In the present case, the pump group velocity in $\mathrm{ZnGeP}_{2}$ is increasingly larger than generated THz phase velocities for photon energies smaller than $\sim 1 \mathrm{eV}$. The leading transient, generated near the back face, appears at a time consistent with the larger pump group velocity. The trailing transient, generated near the front face, appears at a time consistent with the smaller $\mathrm{THz}$ phase velocities. Because tuning of the OPA may introduce small artificial time delays into the data, each trace is time shifted such that the trailing negative peak (blue) is fixed in time for photon energies below $0.9 \mathrm{eV}$. This is consistent with the above explanation: the trailing pulse will travel at the generated $\mathrm{THz}$ phase velocities which remain approximately constant with tuning of the pump photon energy.

\subsubsection{Frequency Domain Mapping \& Modeling}

From the wave equation (Equation (2.1)) and the frequency domain definition of the second-order THz-optical-rectification polarization (Equation (2.11)), Faure et al. [78] express the theoretical emitted field explicitly as a function of THz frequency $\omega$ and position (crystal thickness) $z$ as: 


$$
\begin{aligned}
E_{T H z}(z, \omega)= & \frac{\sqrt{2} \pi \chi^{(2)}(\omega)}{n_{T H z}^{2}-n_{g}^{2}} \tau e^{-\omega^{2} \tau^{2} / 4} E_{o}^{2} \\
& {\left[\frac{1}{2}\left(1+\frac{n_{g}}{n_{T H z}}\right) e^{i \omega n_{T H z} z / c}+\frac{1}{2}\left(1-\frac{n_{g}}{n_{T H z}}\right) e^{\left(-i \omega n_{T H z} z / c\right)}-e^{\left(i \omega n_{g} z / c\right)}\right] . }
\end{aligned}
$$

In obtaining this expression dispersion, absorption, and phase mismatch are all accounted for. The crystal length is assumed to be smaller than the near-infrared and $\mathrm{THz}$ Rayleigh lengths. The second-order susceptibility, $\mathrm{THz}$ index of refraction, and pump group index of fraction are evident in Equation (4.2); $\tau$ is the pump-pulse duration. $E_{o}^{2}$ is proportional to the pump intensity.

This result predicts the generated $\mathrm{THz}$ pulse as a superposition of three waves. The first term exhibits the accumulated phase of a given $\mathrm{THz}$ spectral component after a distance $z$ in the crystal; the negligible second term represents backward propagation. The third exhibits the accumulated phase of the forward propagating pump pulse, traveling at the near-infrared group velocity. Overall variation of generation efficiency is accounted for in the leading multiplier, as is the bandwidth envelope of generation which depends on the pump-pulse duration. The expected effects of phase mismatch in the frequency domain are well described by Equation (4.2). The non-negligible terms show distinct spectral phases determined by the pump group and $\mathrm{THz}$ phase indices respectively. For significant mismatch between these indices interference fringes are predicted in the frequency domain.

To observe and map this fringing, fast Fourier transforms (FFT) of the time-domain traces from Figure 4.5 were performed. These FFTs are plotted in Figure 4.6 (a) and (b) respectively and normalized to the highest peak at a given pump-photon energy/wavelength. A continuous broadband spectrum only occurs where phase matching is approximately satisfied near $1.12 \mu \mathrm{m}$ (110) and $1.28 \mu \mathrm{m}$ (012). While the bandwidth of generation remains approximately constant with pump tuning, fringes begin to appear with a spectral period that increases with diverging pump group velocity and the $\mathrm{THz}$ phase velocity. The periodicity of fringing at a given pump wavelength should be accurately modeled using Equation (4.2) alone; however, other experimental factors affect detection efficiency as a function of $\mathrm{THz}$ frequency. The THz pulse ultimately detected by EO sampling is bandwidth limited in comparison to the 


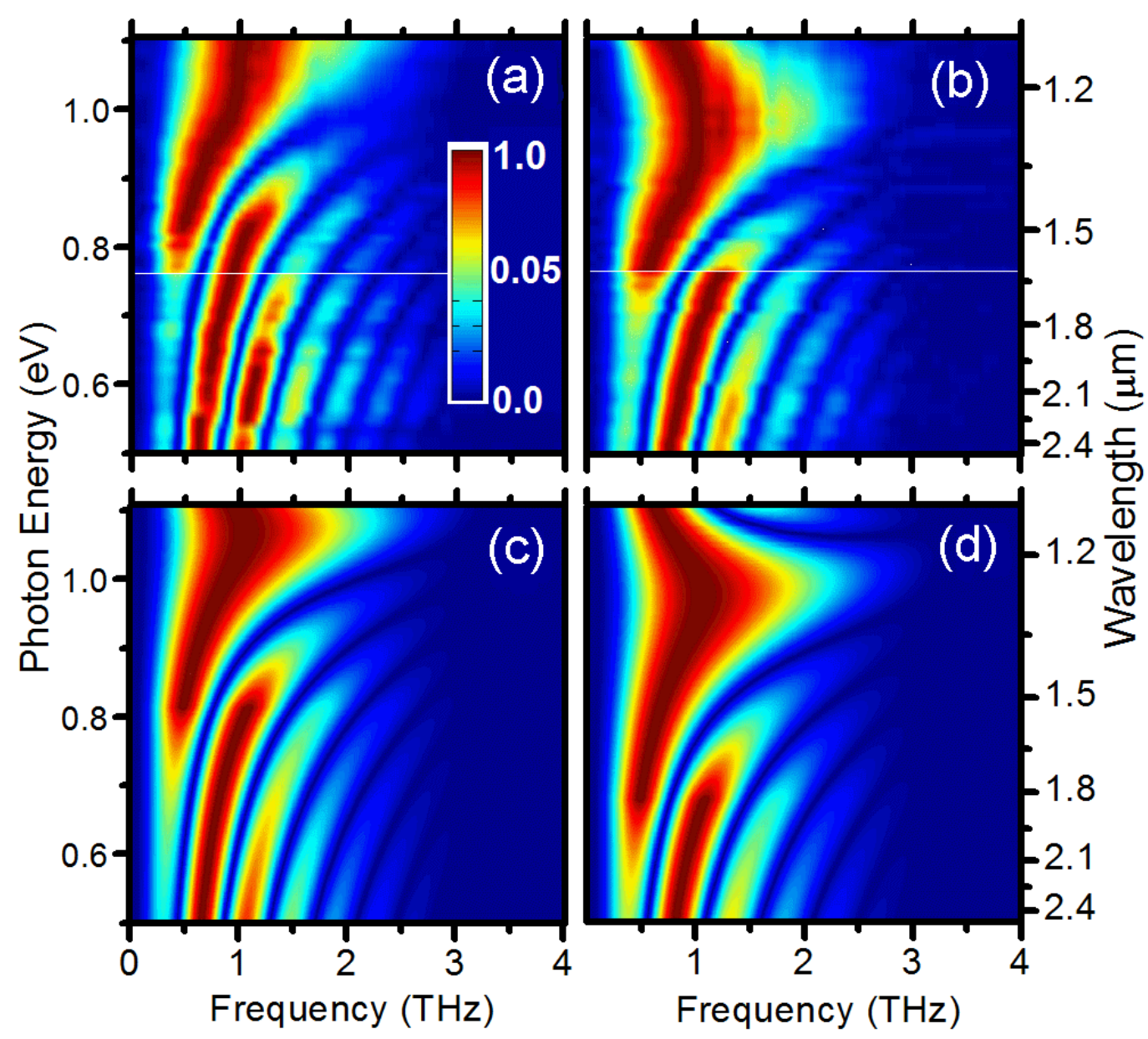

Figure 4.6: (a) Fourier transform of the temporal data for (110), (b) Fourier transform of the temporal data for (012), (c) simulated (110) response and (d) simulated (012) response. All data and calculations are normalized to the highest peak for a given pump wavelength.

generated pulse. This is a result of frequency dependent attenuation in transmitting the $\mathrm{THz}$ pulse from source to detection and the focusing/phase-matching condition at the detection crystal itself. Frequency transfer functions accounting for these experimental realities are therefore defined and applied; see [78].

The first significant transfer function is

$$
T_{\text {diff }}(\omega)=\left(1-\exp \left[-\frac{\tan ^{2}\left(\theta_{\text {col }}\right) z_{R}^{2}}{r_{o}^{2}}\right]\right)^{1 / 2},
$$

which accounts for frequency dependent diffraction of the emitted $\mathrm{THz}$ pulse and the limited collection angle $\left(\theta_{c o l}\right)$ of the collecting off-axis parabolic mirror. This transfer function depends 
on the pump beam spot-size $\left(r_{o}\right)$ and the Rayleigh length of the THz beam: $z_{R}=k r_{o}^{2} / 2$. This function tends to limit the detection of low frequency components which diffract more strongly. The transfer function accounting for overlap of the probe and $\mathrm{THz}$ beams in the EO detection crystal is

$$
\begin{gathered}
T_{\text {overlap }}(\omega)=r_{f o c}(\omega)^{2} /\left(2 r_{f o c}(\omega)^{2}+r_{p}^{2}\right)^{1 / 2} ; \\
r_{f o c}(\omega)=2 c f_{f o c} / \omega r_{\text {in }}(\omega) ; \\
r_{\text {in }}(\omega)=r_{o}\left(1+\frac{f_{\text {coll }}^{2}}{z_{R}(\omega)^{2}}\right) .
\end{gathered}
$$

$r_{f o c}(\omega)$ is the frequency dependent spot-size of the $\mathrm{THz}$ transverse profile at the EO detection crystal, $r_{p}$ is the $1 / \mathrm{e}^{2}$ probe radius, while $f_{\text {coll }}$ and $f_{\text {foc }}$ are the focal lengths of the collection and focusing off-axis parabolic mirrors; these were identical at $75 \mathrm{~mm}$ in the experimental setup. The most crucial transfer function is

$$
T_{\mathrm{det}}\left(L_{\mathrm{det}}, \omega\right)=T_{i n} \frac{\exp \left[i L_{\mathrm{det}}\left(n_{T H z}-n_{g}\right) \omega / c\right]-1}{i \omega / c\left(n_{T H z}-n_{g}\right)} \exp \left(-\tau_{p}^{2} \omega^{2} / 4\right),
$$

which defines the detection bandwidth of EO sampling. It is a function of the crystal length ( $L_{\text {det }}$ ), phase mismatch between probe-group velocity and THz-phase velocities $\left(n_{T H z}-n_{g}\right)$, frequency dependent reflectivity $\left(T_{\text {in }}(\omega)=2 /[\sqrt{\varepsilon}+1]\right)$, and the probe pulse duration $\left(\tau_{p}\right)$.

The observed frequency domain fringing and detected bandwidth, resulting from diffraction, focusing, and electro-optic sampling were modeled with Equation (4.2) multiplied by the transfer functions described above.

$$
E_{T H z}^{D e t} \propto E_{T H z}(z, \omega) T_{\text {diff }}(\omega) T_{\text {overlap }}(\omega) T_{\text {det }}(\omega)
$$

The result of this model is shown in Figure 4.6 (c) and (d). Good agreement between the model and experimental data is achieved. Slight error in the fringe position/spacing is primarily attributable to limited accuracy of measured crystal thicknesses ( $\sim \mu \mathrm{m}$ error) or inaccuracy of the literature dispersion relations $[97,118]$. According to the model, the attenuation in detected 
signal at low $\mathrm{THz}$ frequencies is primarily due to diffraction of the $\mathrm{THz}$ pulse at the source. Input parameters for the calculation include measured crystal thicknesses of $3.01 \mathrm{~mm}(110)$ and 3.03 $\mu \mathrm{m}$ (012), pump-pulse duration of $100 \mathrm{fs} F W H M, 1 / \mathrm{r}^{2}$ pump radius of $0.85 \mathrm{~mm}$, probe wavelength of $800 \mathrm{~nm}$, probe-pulse duration of $300 \mathrm{fs}$, and detection crystal thickness of 0.5 $\mathrm{mm}$. All of these parameters are in reasonable agreement with experimental conditions with the exception of the probe pulse duration, expected to be closer to $100 \mathrm{fs}$. Supposing that no unexpectedly dispersive optical element was introduced into the probe path this discrepancy between model and experiment is unresolved. It should, however, be noted that the probe pulse duration only shifts the $\mathrm{THz}$ spectral peak and does not affect the frequency domain fringing the limit in detection bandwidth. The limit in detector bandwidth is primarily determined by the EO crystal thickness and was accurately modeled for any probe duration.

\subsection{Chapter Summary}

The discussion, calculations, and data presented in this chapter demonstrate the paramount importance of accounting for phase mismatch in THz optical rectification generally, while exploring the effects of phase matching in $\mathrm{ZnGeP}_{2}$ and other chalcopyrite semiconductors. The parameters and phase-matching dispersion maps provided in Section 4.1 will provide valuable information to researchers considering chalcopyrite semiconductors as $\mathrm{THz}$ emitters. These parameters and calculations indicate that $\mathrm{CdGeP}_{2}$ should exhibit higher generation efficiency than $\mathrm{ZnGeP}_{2}$ and $\mathrm{GaAs}$ as well as improved phase matching at the $1.55 \mu \mathrm{m}$ fiber laser wavelength.

The data and model presented in Section 4.2 demonstrate the effects of phase mismatch on emission efficiency and generated THz waveforms. The data verify that the (110) and (012) $\mathrm{ZnGeP}_{2}$ planes exhibit distinct optimal pump wavelengths due to birefringence. These results corroborate the qualitative and quantitative predictions of existing models describing the form of $\mathrm{THz}$ field amplitudes generated by optical rectification as a function of phase mismatch. The data are also consistent with earlier work analyzing waveforms at three distinct pump frequencies and applying a simple model [132], but represent the first experimental 
quasi-continuous mapping of $\mathrm{THz}$ optical rectification transients in the time and frequency domain as a function of phase mismatch. 


\section{Chapter 5 Optical Rectification in ZnGeP 2 : Length \& Intensity Scaling}

Data describing the pump-intensity efficiency scalability of $\mathrm{THz}$ optical rectification are of interest from a practical engineering standpoint, but general implications may also be drawn from an accurate understanding of factors limiting this scalability in similar materials. In this chapter, data are presented showing a rollover in $\mathrm{THz}$ optical rectification efficiency at high pump intensities for (012)-cut $\mathrm{ZnGeP}_{2}$ crystals of three thicknesses: $0.3 \mathrm{~mm}, 0.9 \mathrm{~mm}$, and 3.0 $\mathrm{mm}$. Qualitative argument and modeling support three mechanisms governing this rollover including pump-depletion due to multiphoton excitation of carriers across the band-gap, increased free-carrier absorption (FCA) resulting from these excitations, and self-phase modulation (SPM); the final mechanism is only evident in the thickest crystal. The mechanisms of multiphoton pump depletion and resulting free-carrier absorption are consistent with the findings of Harrel et al. [133] who studied THz optical rectification in ZnTe at $800 \mathrm{~nm}$, for which two-photon absorption (2PA) is the dominant multiphoton absorption (MPA) process. In contrast, phase-matched pump photon energies in $\mathrm{ZnGeP}_{2}$ are below the onset of $2 \mathrm{PA}$; the pump pulse is primarily subject to 3PA. The onset of significant 3PA occurs at higher pump intensities than 2PA; see Section 2.1.1.3. While Harrel et al. isolated the effects of 2PA and FCA, they did not reach sufficiently high pump fluence and crystal thickness to observe additional effects resulting from SPM in ZnTe.

For data collection, the pump beam $(1.28 \mu \mathrm{m})$ impinged the samples at normal incidence with polarization purely e-wave in the (012) cut plane for maximum generation efficiency. Phase matched pump wavelengths in this orientation are well within the most stable region of the OPA tuning range. The pump was configured in a loose focusing condition (1/e ${ }^{2}$ spot-radius of $1.18 \mathrm{~mm}$ ) by placing the sample in the beam path about half the distance before the focus of a $150 \mathrm{~mm}$ lens. For repeatability when switching between samples, the back face of each sample was referenced to a fixed straight edge without altering the alignment of the setup. The same razor straight edge was translated with a micrometer to determine the spot 
size in the crystal. The OPA pulse duration was measured by intensity autocorrelation. The peak pump intensity was adjusted by reducing the incident pump power using neutral-density filters of measured/calibrated transmission at $1.28 \mu \mathrm{m}$.

\subsection{Terahertz Waveforms: Low \& High Pump Intensity}

Figure 5.1 (a) and (b) show representative detected time-domain traces of $\mathrm{THz}$ pulses generated from three crystal thicknesses. Figure 5.1 (a) shows transients at low intensity pumping $\left(<10 \mathrm{GW} / \mathrm{cm}^{2}\right)$ and defines the field amplitudes: $E_{1}, E_{2}$, and $E_{3}$. Figure 5.1 (b) shows detected THz traces for high pump intensity: $35 \mathrm{GW} / \mathrm{cm}^{2}$ after reflection from the crystal surface (i.e. internal peak intensity). For reference, a time-domain trace is included in Figure 5.1 (b) for low power pumping $\left(<10 \mathrm{GW} / \mathrm{cm}^{2}\right)$ of the $0.9 \mathrm{~mm}$ thick crystal (dotted line). All $\mathrm{THz}$ waveforms are normalized to $E_{1}$ for best comparison of the $\mathrm{THz}$ waveforms: as will be shown in the next section and as demonstrated elsewhere [133], the effects of MPA and FCA on this leading peak is small. We observe a nonlinear relationship between peak-pump intensity and $E_{1}$ only at high pump intensities and the thickest crystal; see Figure 5.3 (a). Fourier transforms of the $\mathrm{THz}$ transients for low intensity pumping and high intensity pumping are shown in Figure 5.1 (c). The thinnest crystal at low pump intensity provides a reference spectrum: minimal effect from pump dispersive time-domain stretching and no effects from MPA-induced THz FCA or SPM.

In general, stretching of the pump pulse leads to a stretching of the generated $\mathrm{THz}$ pulse [78]. Stretching of the $\mathrm{THz}$ pulse may be quantified by the difference between peak times of $E_{2}$ or $E_{3}$ relative to the peak time of $E_{1}$. The low pump intensity THz waveforms (Figure 5.1 (a)) exhibit minimal stretching with greater crystal thickness indicative of minimal pump pulse stretching. The small but significant stretching observed in thicker crystals is readily explained in terms of linear group-delay dispersion of the pump pulse and linear absorption of the generated $\mathrm{THz}$ pulse. As a result of this stretching, a small but significant shift to lower frequencies is observable in the Fourier transform of low intensity $\mathrm{THz}$ transients in the thick crystal (not shown). 


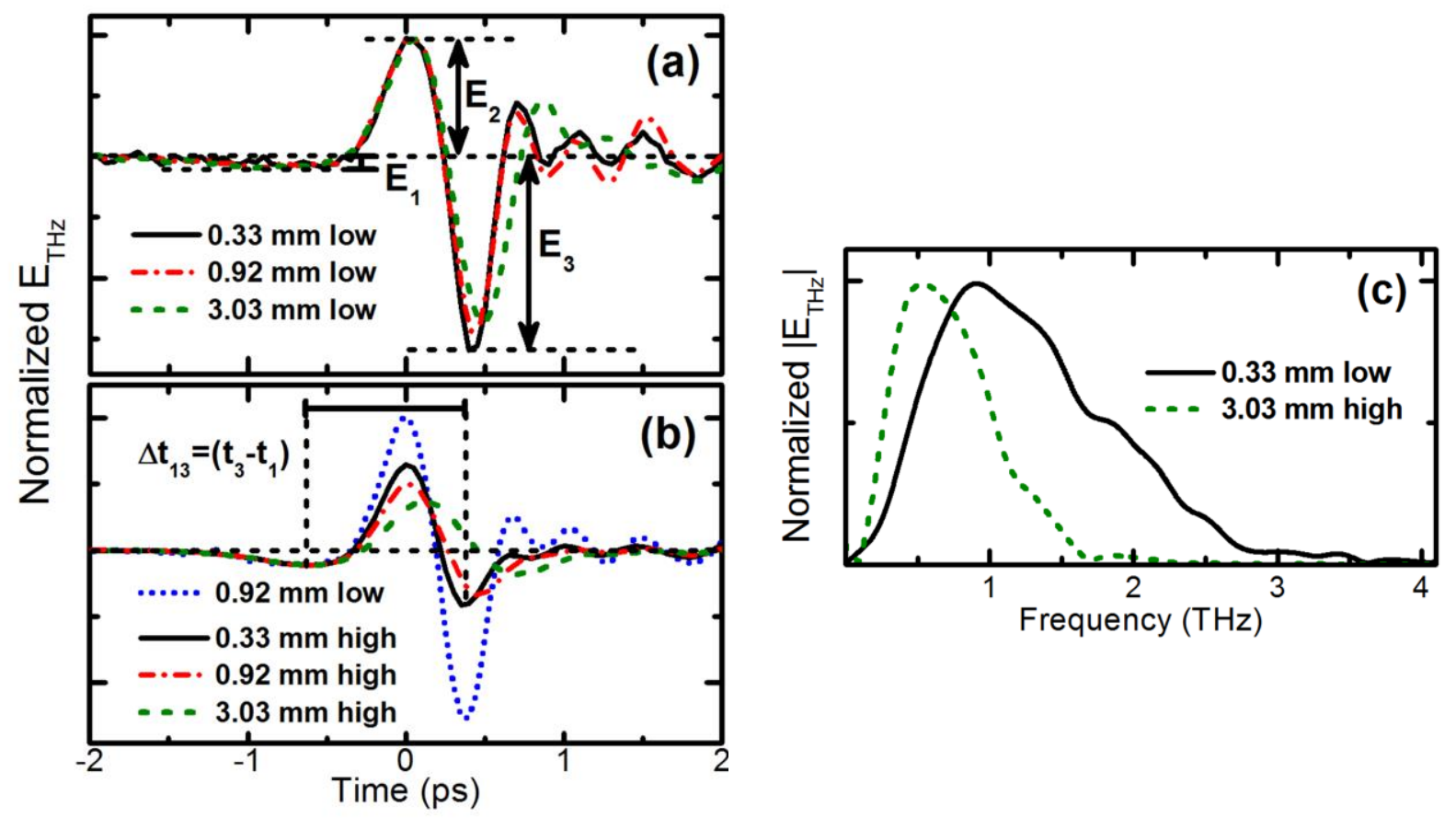

Figure 5.1: Detected THz transients for (a) low $\left(<10 \mathrm{GW} / \mathrm{cm}^{2}\right)$ pump intensity and (b) high $\left(35 \mathrm{GW} / \mathrm{cm}^{2}\right)$ pump intensity with crystal thicknesses of $0.33 \mathrm{~mm}, 0.92 \mathrm{~mm}$, and $3.03 \mathrm{~mm}$ normalized to the leading peak. (c) Broadest and narrowest generation bandwidths: $0.33 \mathrm{~mm}$ crystal with low intensity pumping and $3.03 \mathrm{~mm}$ thick crystal with high intensity pumping.

More significant changes are apparent in the high intensity waveforms; see Figure 5.1 (b). Comparing the $0.92 \mathrm{~mm}$ low intensity transient (dotted) to the high intensity transient (solid), a strong change in the relative amplitudes of $E_{2}$ and $E_{3}$ relative to $E_{1}$ is apparent with $E_{3}$ the most strongly affected. Slight stretching of the $\mathrm{THz}$ pulse is apparent for the high intensity $0.92 \mathrm{~mm}$ transient in the form of a small shift in $E_{2}$ later in time. When the crystal length is increased to $3.03 \mathrm{~mm}$ both $E_{2}$ and $E_{3}$ show a significant shift relative to $E_{1}$.

The observed increase in attenuation of the $\mathrm{THz}$ pulse at later times is an anticipated effect of MPA-induced FCA. Harrel et al. observed and modeled this time-dependent increase in FCA due to MPA using a straightforward model, which is instructive [133]. The model assumes instantaneous excitation of free carriers as a function of time-dependent pulse intensity: $N_{e}(t) \propto \int_{-\infty}^{\infty} I_{o p t}^{2}\left(t^{\prime}\right) d t^{\prime}$, where $N_{e}(t)$ is the electron number density. This assumption leads to an attenuation function, $\exp \left[-N_{e}(t) e_{\text {scale }}\right] ; e_{\text {scale }}$ is a fitting factor. Their model for low and high pump fluence is shown in Figure 5.2. The model demonstrates the effect of a time-varying 
carrier density on the generated $\mathrm{THz}$ pulse. While the qualitative behavior of the $\mathrm{THz}$ waveforms presented here, in moving from low intensity to high intensity, is in agreement with their observations and model, no shifting of the peak times with pump intensity is observed with increasing pump intensity. Based on the arguments to be presented, a transition to a regime of significant SPM takes place as the crystal length is increased. Qualitatively, SPM distorts and stretches the pump pulse resulting in a stretching of the $\mathrm{THz}$ pulse in the time domain. A stretched $\mathrm{THz}$ pulse shows slower time evolution and lower spectral frequencies. As is elucidated in the following section, the reduced spectral bandwidth observed in Figure 5.1 (c) for the case of a thick crystal at high intensity is primarily a result of 3PA induced FCA and SPM.

\subsection{Peak THz Field Amplitudes \& Analysis}
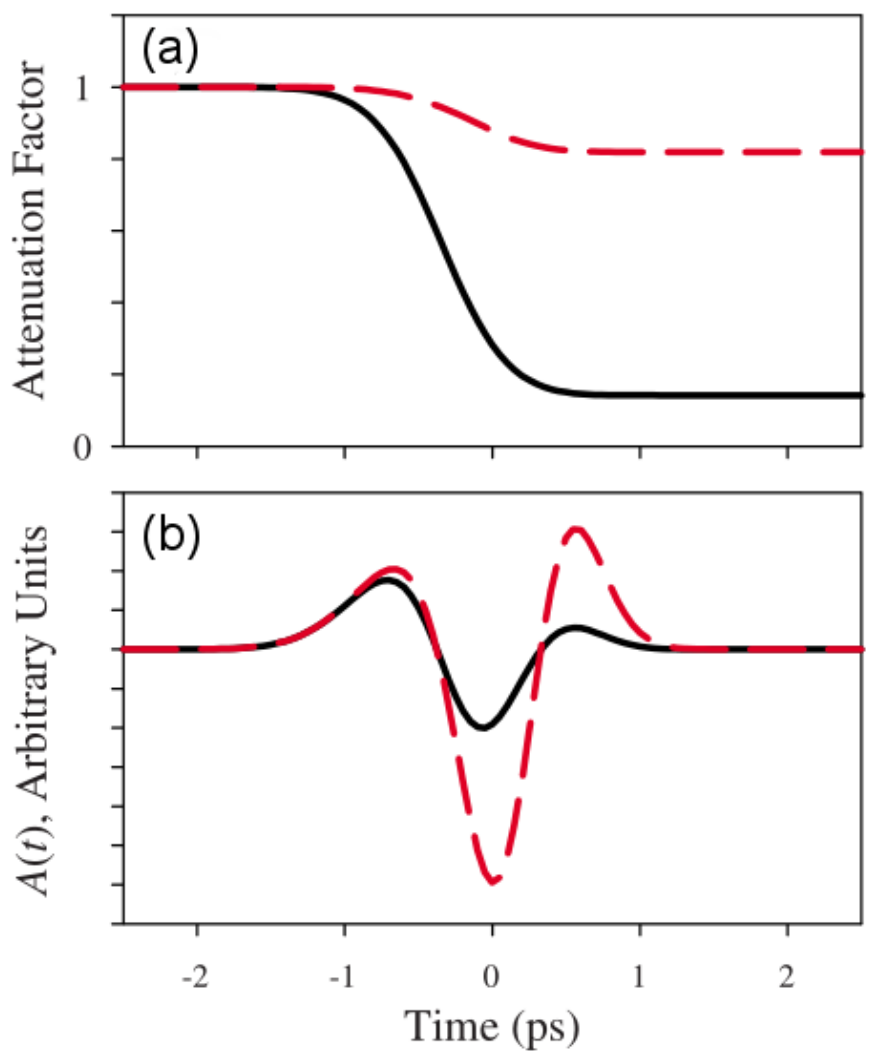

Figure 5.2: (a) Attenuation factors resulting from free-carrier absorption for high (solid black) and low (dashed red) pump fluence. (b) Effect of the attenuation factors on the generated THz pulse for low and high pump fluence. Figure reproduced from [127]. 
Figure $5.3(\mathrm{a}),(\mathrm{b})$, and (c) show the dependence of $E_{1}, E_{2}$, and $E_{3}$ (see Figure $5.1(\mathrm{a})$ ) on the internal peak-pump intensity (i.e. intensity after reflection loss from the front surface). The significant difference in the character of these plots underlines the nonuniform manner in which pump depletion due to 3PA and the resulting FCA affect the generated THz pulse; that is, a stronger rollover in emission efficiency is observed for later peaks for all three crystal thicknesses.

As expected, the data are linear at low pump intensities. Below the intensity onset of significant MPA and assuming perfect phase matching, the pump and $\mathrm{THz}$ pulses will experience linear absorption according to Beer's law: $d I(z) / d z=-\alpha_{\text {opt }} I(z)$, $d E_{T H z}(z) / d z=-\left(\alpha_{T H z} / 2\right) E_{T H z}(z)$. Also, in general $d E_{T H z} / d z \propto d_{e f f} I(z)$; see Section 2.1.1.2. $I$ is the pump intensity, $E_{T H z}$ is the THz electric field amplitude, $\alpha_{o p t}$ and $\alpha_{T H z}$ are the optical and $\mathrm{THz}$ linear absorption coefficients, and $z$ is the distance of pulse propagation within the generating crystal. The slope of the linear dependence between $E_{T H z}$ and $I$ is then defined by $\alpha_{o p t}, \alpha_{T H z}, d_{e f f}$, and the length of the crystal $L_{c}$. The slope will increase with crystal thickness up to some limit set by the linear absorption coefficients.

A transition to nonlinear dependence at sufficiently high pump intensity is consistently observed for peaks $E_{2}$ and $E_{3}$. This is qualitatively expected, for pump depletion due to MPA and resulting THz FCA, based on discussion in the previous section. MPA pump depletion can be modeled using a generalized Beer's Law with coefficients for 2PA $(\beta), 3 \mathrm{PA}(\gamma)$, and higher order coefficients; see Section 2.1.1.3. At $1280 \mathrm{~nm} 2 \mathrm{PA}$ is negligible compared to 3PA in $\mathrm{ZnGeP}_{2}$, so we have:

$$
\begin{gathered}
\frac{d I(z)}{d z}=-\alpha_{o p t} I(z)-\gamma I^{3}(z) \\
\frac{d E_{T H z}(z)}{d z}=C_{\text {scale }} d_{e f f} I(z)-\frac{\alpha_{T H z}}{2} E_{T H z}(z) .
\end{gathered}
$$



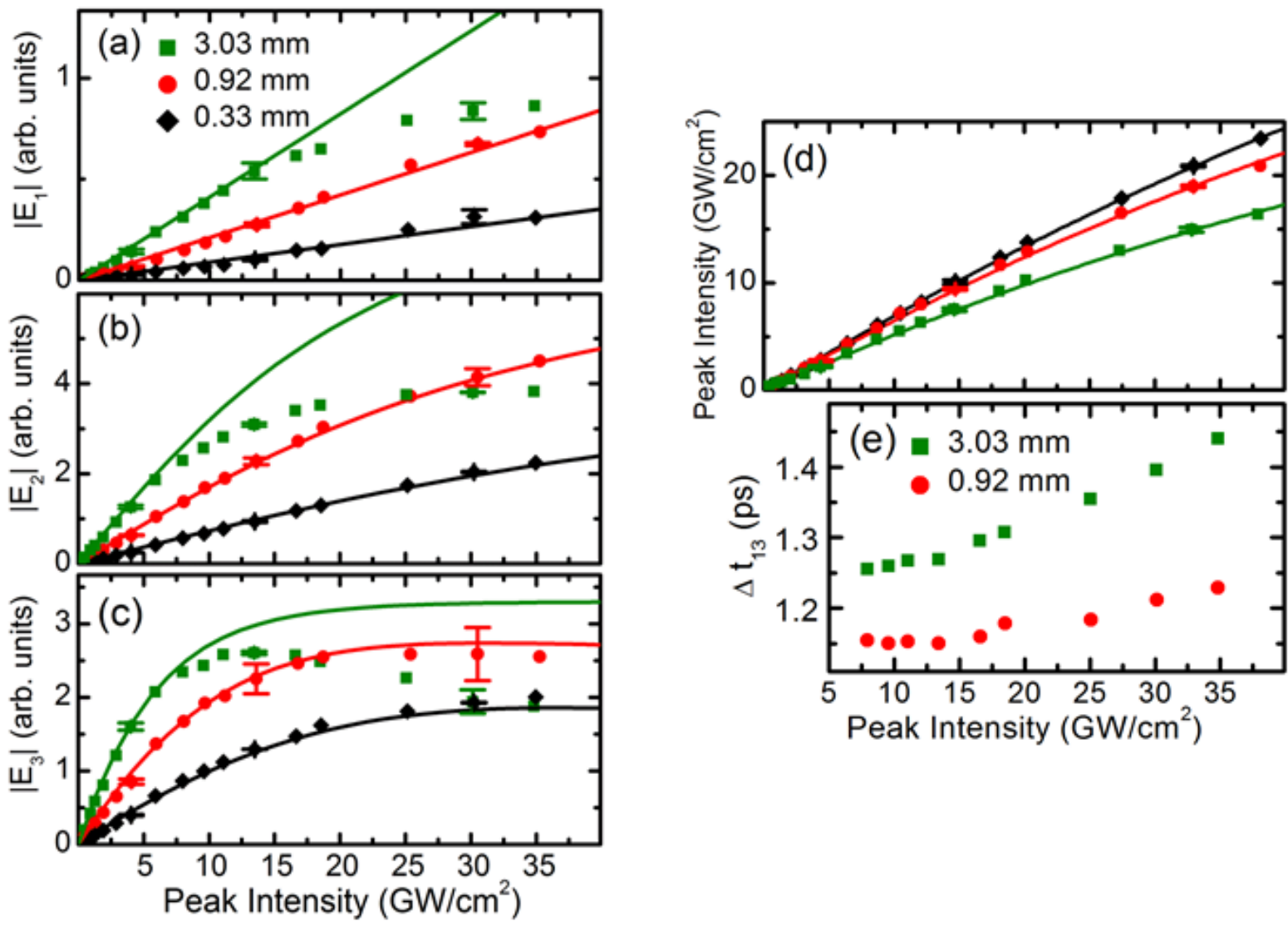

Figure 5.3: Electric-field amplitude of the Leading peak, $E_{l}$, (a) and peak-to-peak, $E_{P P}$, (b) of detected THz time-domain transients as a function of Incident peak pulse intensity, just after reflection from the crystal surface. In (a) solid lines represent linear fits to the data with only points below $15 \mathrm{GW} / \mathrm{cm}^{2}$ included in the fit for the $3.03 \mathrm{~mm}$ dependence. In (b) the lines are theoretical fits to the data accounting for pump depletion only. (c) Pump depletion: transmitted peak pulse intensity as a function of incident peak pulse intensity. The data are fit using equation (5) to determine an effective $\gamma$ value.

$C_{\text {scale }}$ is an adjustable parameter with the effect of scaling the simultaneous solution of (5.1) and (5.2). MPA-induced FCA, which is neglected in these equations, does not affect the $\mathrm{THz}$ field amplitude in the time domain uniformly; see the previous section. That is, FCA is stronger at later times across the $\mathrm{THz}$ pulse with increasing photoexcited free-carrier density. As a result, the generated $\mathrm{THz}$ electric field amplitude must be modeled at a given moment in time: $\alpha_{\mathrm{TH} z}$ becomes a function of $I(z)$ and time relative to the pump pulse [133]. To account for additional linear $\mathrm{THz}$ absorption resulting from photoexcited free-carriers, a modifying term is added the $\mathrm{THz}$ absorption term of equation (5.2). Assuming the strength of $\mathrm{THz}$ absorption is directly proportional to the free carrier concentration [134]: 


$$
\frac{d E_{T H z}(z)}{d z}=C_{\text {scale }} d_{\text {eff }} I(z)-\left\{\frac{\alpha_{T H z}}{2}+a_{F C A}\left(\alpha_{o p t} I+\frac{1}{3} \gamma I^{3}(z)\right)\right\} E_{T H z}(z)
$$

The term modifying $\alpha_{T H z}$ involves $a_{F C A}$, a second adjustable parameter compensating for the strength of FCA at a given time across the generated THz pulse.

To obtain realistic model values for $\alpha_{o p t}$ and $\gamma$, and to narrow the number of free parameters, pump depletion was measured directly by observing transmission through a sample over the full range of pump intensities and for each crystal thickness. Solving Equation (5.1) for a pulsed Gaussian beam while accounting for reflectivity from both surfaces gives a transmittance of [80]:

$$
\begin{gathered}
T=\frac{(1-R)^{2} e^{-\alpha L_{c}}}{\sqrt{\pi} P_{o}} \int_{-\infty}^{\infty} \ln \left[\sqrt{1+P_{o}^{2} e^{-2 t^{2}}}+P_{o} e^{-t^{2}}\right] d t, \\
P_{o}=\left[2 \gamma(1-R)^{2} I_{o}^{2} L_{e f f}\right]^{1 / 2}, \\
L_{e f f}=\left[1-\exp \left(-2 \alpha L_{c}\right)\right] / 2 \alpha .
\end{gathered}
$$

Here $R$ is the reflectance calculated from the index of refraction and $I_{o}$ is the incident intensity. The integral accounts for temporal variation, assuming a Gaussian pulse. Pumpdepletion data are shown in Figure 5.3 (d) where the horizontal axis gives internal peak-pump intensity at the entrance face and the vertical axis gives the internal peak-pulse intensity at the exit face of the crystal. The pump-spot size, pulse duration and power range were identical for this depletion measurement and the emission data displayed in Figure 5.3 (a)-(c). For powers < $10 \mathrm{GW} / \mathrm{cm}^{2}$ a solution to Beer's law provides a simultaneous fit for all three linear slopes for $\alpha_{\text {opt }}=1.07 \mathrm{~cm}^{-1}$. Solutions to equation (5.4) were then applied to model the full peak-intensity range for each thickness. The average modeled value of $\gamma$ for the three thicknesses was 0.01

Table 2. Modeling parameters for intensity-dependent $\mathrm{THz}$ field amplitude features extracted from the transients for various ZGP-(012) crystal thicknesses.

\begin{tabular}{ccccc}
\hline & \multicolumn{3}{c}{$C$ (Arb. Units) } & $\begin{array}{c}\alpha_{\mathrm{FCA}}(\mathrm{t}) \\
\end{array}$ \\
\cline { 2 - 4 } & $0.33 \mathrm{~mm}$ & $0.92 \mathrm{~mm}$ & $3.03 \mathrm{~mm}$ & $\left(\mathrm{~cm}^{2} / \mathrm{GW}\right)$ \\
\hline$E_{1}$ & 22.5 & $22.5 / 1.17$ & $22.5 / 1.41$ & 0 \\
$E_{2}$ & 190 & $190 / 1.17$ & $190 / 1.41$ & 0 \\
$E_{3}$ & 260 & $260 / 1.17$ & $260 / 1.41$ & 0.33 \\
\hline
\end{tabular}


$\mathrm{cm}^{3} / \mathrm{GW}^{2}$. Best fits for the three crystal thicknesses are shown as solid lines in Figure $5.3(\mathrm{~d})$ and the fitting parameters are summarized in Table 2.

The $\alpha_{T H z}$ value of $3.5 \mathrm{~cm}^{-1}$ (at $1.5 \mathrm{THz}$ ), measured by THz time-domain spectroscopy, is applied in modeling the $\mathrm{THz}$ emission data; see Section 2.4.1.

The low power linear slopes were not accurately fit for all three crystal thicknesses using a single value of $C_{\text {scale }}$. For the $3.03 \mathrm{~mm}, 0.92 \mathrm{~mm}$, and $0.33 \mathrm{~mm}$ thick crystals the values used for all peaks were $C_{\text {scale }}=1,0.855$, and 0.708 respectively. Alternatively the slopes of all crystal thicknesses could be fit using a significantly larger value of $\alpha_{o p t}=4.5 \mathrm{~cm}^{-1}$. Using either approach the results were approximately identical; the approach using separate scales required a slightly higher value of $a_{F C A}\left(0.33 \mathrm{~cm}^{2} / \mathrm{GW}\right.$ vs $\left.0.25 \mathrm{~cm}^{2} / \mathrm{GW}\right)$ to provide a match to the data within error. The discrepancy is attributable to small but non-negligible pulse stretching, reducing the peak intensity, due to linear group-delay dispersion. Non-ideal focusing for significant source thickness may also affect focusing at the detector: not every point in a thick crystal resides at the exact focal point of the collection off-axis parabolic.

A standard measure of the significance of linear dispersion is the dispersion length:

$$
L_{D}=\tau_{o}^{2} /\left(\frac{d^{2} k}{d \omega^{2}}\right)_{\omega_{o}}
$$

$\tau_{o}$ is the initial pump pulse duration and $k$ is the wavevector. Linear dispersion results in time domain chirp and broadening of the pump pulse. For a pump wavelength of $1280 \mathrm{~nm}$ and initial pulse duration of $120 \mathrm{fs}$ FWHM in ZGP $L_{D}$ is $12 \mathrm{~mm}$. For a transform limited Gaussian pulse the pulsewidth doubles at $0.6 L_{D}$. This suggests that for the 3-mm thick ZGP the excitation pulse has been stretched by $\sim 25 \%$, which is non-negligible and is expected to be the cause of the lower-than-expected slopes for thicker crystals.

The pump intensity data for peak field amplitudes $E_{1}, E_{2}$, and $E_{3}$ are modeled with the above parameters and simultaneous numerical solution of Equations (5.1) and (5.3). For all peaks the model accurately describes the efficiency rollover for the thinner $0.3 \mathrm{~mm}$ and $0.9 \mathrm{~mm}$ crystals, but the $3 \mathrm{~mm}$ thick data diverges from the model at high pump intensities. 
$E_{1}$ is linear within error for the $0.3 \mathrm{~mm}$ and $0.9 \mathrm{~mm}$ crystals over the full intensity range indicating that neither 3PA-induced pump depletion and FCA measurably affect this leading peak. $a_{F C A}$ and $\gamma$ were both set to zero in the model. Distinct behavior is observed for $E_{1}$ from the $3 \mathrm{~mm}$ crystal which shows a significant rollover at high pump intensities. 3PA pump depletion alone $\left(\gamma=0.01 \mathrm{~cm}^{3} / \mathrm{GW}^{2}\right)$ is sufficient to explain the mild rollover in $E_{2}$ observed for both thinner crystals. Both 3PA $\left(\gamma=0.01 \mathrm{~cm}^{3} / \mathrm{GW}^{2}\right)$ and FCA $\left(a_{F C A}=0.25\right)$ were necessary to model the rollover in the final peak for the two thin crystals.

The model predicts a considerably weaker rollover for the $3 \mathrm{~mm}$ thick crystal than is observed for all peaks. Figure 5.3 (e) quantifies the THz pulse stretching, discussed qualitatively in the previous section, for the $0.9 \mathrm{~mm}$ and $3 \mathrm{~mm}$ crystals. This broadening of the generated $\mathrm{THz}$ pulse with pump intensity is indicative of pump pulse stretching with increasing intensity. This is consistent with the previously discussed effects of SPM. Distortion of the pump pulse

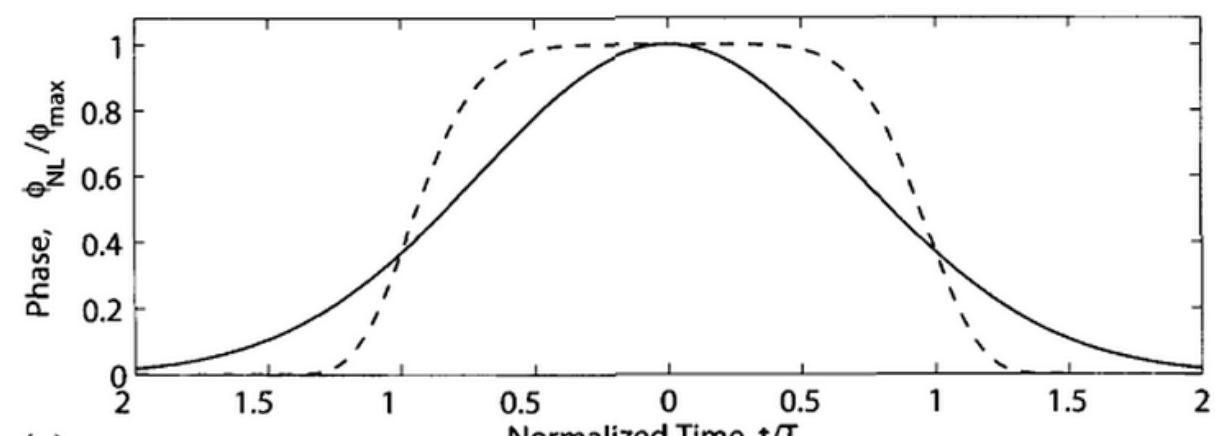

(a)

Normalized Time, $\mathrm{t} / \mathrm{T}_{0}$

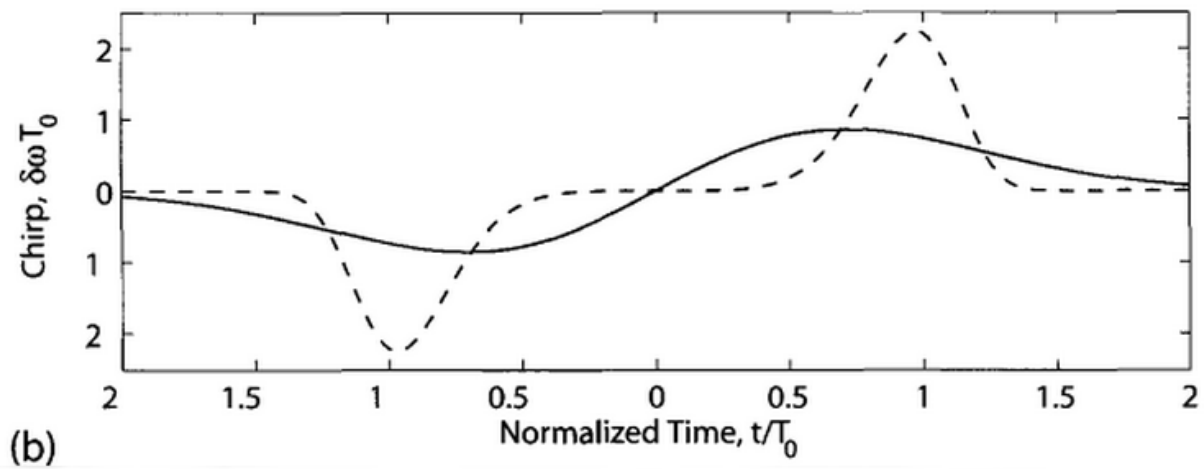

Figure 5.4: (a) The instantaneous phase normalized to the peak phase as a function of time. (b) The frequency chirp as a function of time. $T_{0}$ is the pulse duration and $t=0$ is at the peak pulse intensity. The solid line is for a Gaussian intensity profile and the dashed line is for a flat-top pulse profile. Figure reproduced from [130]. 
may diminish the pump-pulse peak intensity and the efficiency of THz optical rectification. While SPM is not here incorporated into a model describing efficiency of $\mathrm{THz}$ generation, some approximation of the onset of significant SPM and its significance relative to linear dispersion should be considered.

SPM results from time variation of the refractive index with the pump intensity profile: $n(t)=n+n_{2} I(t)=n+\Delta n(t)$, where $n_{2}$ is the nonlinear index of refraction. The time-dependent index tends to alter a pulse from its original state: pulse chirp and in the time domain plus spectral broadening and formation of "wings" in the frequency domain; see [135] for example spectra. The pulse chirp and spectral broadening tend to enhance the effects of linear groupdelay dispersion, stretching the pulse. Assuming an instantaneous material response, the effect of SPM at each time across a pulse can be quantified by the phase shift of the electric field, $\phi_{N L}(t)$ :

$$
\begin{gathered}
\phi(t)=\omega_{o} t-k x=\omega_{o} t-\frac{\omega_{o}}{C} n(t) x=\omega_{o} t-\frac{\omega_{o}}{C} n x+\phi_{N L}(t) ; \\
\phi_{N L}(t)=-\frac{\omega_{o}}{C} n_{2} I(t) x .
\end{gathered}
$$

Considering the definition of instantaneous angular frequency, it is clear that the time-varying index introduces a time-varying frequency across the pulse

$$
\omega(t)=d \phi(t) / d t=\omega_{o}+\delta \omega=\omega_{o}-\frac{\omega_{o}}{C} n_{2} \frac{d I(t)}{d t} \chi
$$

From Equations (5.6) and (5.7) it is clear that the nonlinear phase varies with the pulse intensity profile and the instantaneous frequency is defined by the time derivative of the profile. $\phi_{N L}$ and $\delta \omega$ are plotted in Figure 5.4 for Gaussian and flat-top pulse profiles [136].

A standard effective propagation length, similar to the dispersion length, estimating the significance of SPM effects can be defined at which $\phi_{N L}$ at the peak of the pulse equals 1 radian [80]:

$$
L_{N L}=\frac{c}{\omega_{o} n_{2} I_{o}}
$$

Comparing this characteristic length with the dispersion length gives the relative significance of SPM and linear dispersion effects. SPM tends to amplify this time-domain stretching with 

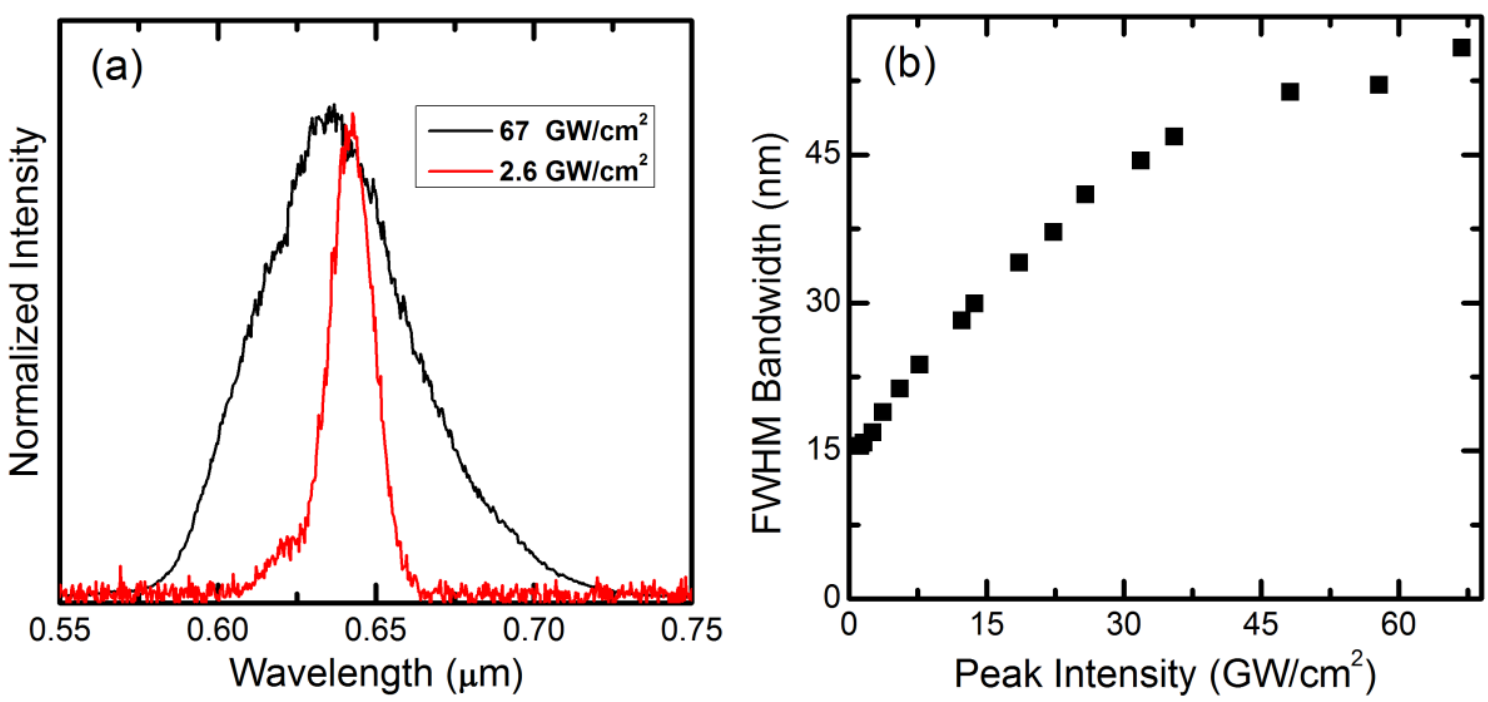

Figure 5.5: (a) Second-harmonic generation spectra of the pump pulse after passing through a $3 \mathrm{~mm}$ thick $\mathrm{ZnGeP}_{2}$ source at low peak intensity and high peak intensity. (b) Second-harmonic generation spectral fullwidth-half-maximum as a function of pump-pulse peak intensity.

additional chirp. Assuming $n_{2}=40 X 10^{-15} \mathrm{~cm}^{2} / \mathrm{W}$ [36] and an optical peak intensity of 40 $\mathrm{GW} / \mathrm{cm}^{2}$, near the maximum intensities reached in the these experiments, $L_{N L}$ is $1.3 \mathrm{~mm}$. As determined above, $L_{D}$ is $12 \mathrm{~mm}$. The significant difference between these numbers suggests that linear dispersion should produce small but significant broadening and chirp in the pump pulse with increasing crystal thickness while SPM should become increasingly dominant for thicker crystals at higher intensity pumping, contributing to stretching and frequency-domain distortion of the excitation pulse. Specifically, the effects of SPM on THz emission should be readily evident in the $3 \mathrm{~mm}$ thick crystal.

To experimentally corroborate the presumed presence and effects of SPM, the spectral bandwidth of the exit pump pulse, passing through the $3 \mathrm{~mm}$ thick crystal, was measured as a function of pump intensity using a $0.5 \mathrm{~mm}$ thick BBO crystal to frequency double the pump into a spectrometer with detection range in the visible. The second-harmonic spectral bandwidth of the exiting pump pulses, which will qualitatively mirror that of the fundamental, increased from $15-50 \mathrm{~nm}$ between $0 \mathrm{GW} / \mathrm{cm}^{2}$ and $40 \mathrm{GW} / \mathrm{cm}^{2}$. This spectral broadening also points to significant SPM of the pump pulse. Given the calculated nonlinear length $L_{N L}$ (above), indicating that SPM should become significant for $\mathrm{ZnGeP}_{2}$ crystal lengths greater than $1.3 \mathrm{~mm}$ 


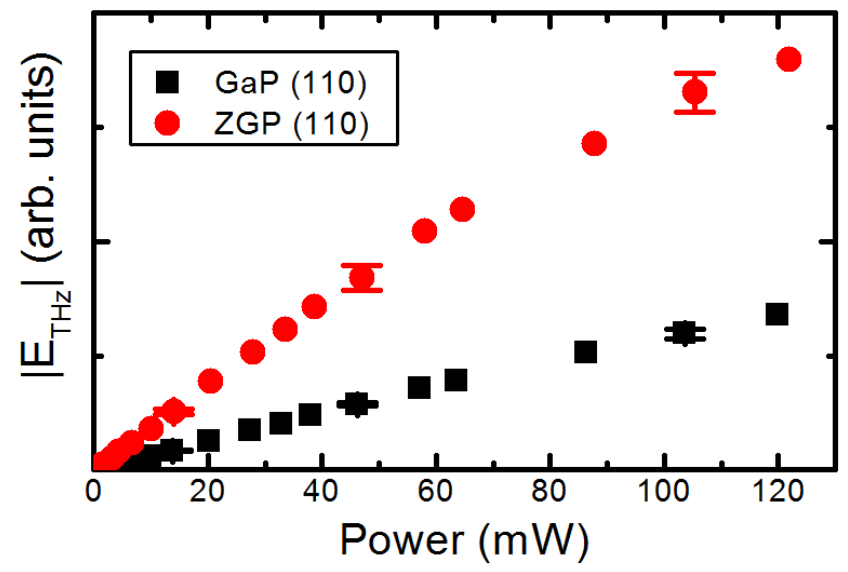

Figure 5.6: Peak-to-peak THz emission as a function of incident pump power from $0.31 \mathrm{~mm}$ thick (110)-cut GaP and $0.33 \mathrm{~mm}$ thick (110)-cut $\mathrm{ZnGeP}_{2}$; data for both crystals was collected in the ooe pump and emission geometry.

at $40 \mathrm{GW} / \mathrm{cm}^{2}$, this spectral broadening is expected in the $3 \mathrm{~mm}$ crystal. These evidences in addition to the divergence of emission data from the model suggest that SPM significantly diminishes the efficiency of $\mathrm{THz}$ generation in thick crystals and at high pump intensities. Thus, in addition to MPA induced FCA, self-phase modulation appears to pose an additional limitation to the intensity scalability of THz-optical-rectification emission efficiency, depending on the size of the nonlinear index of refraction and crystal thickness.

Finally, to directly verify the superior efficiency of $\mathrm{THz}$ optical rectification from $\mathrm{ZnGeP} 2$ to that of GaP, as expected based on the relative second-order nonlinear coefficients, the emission from (110)-cut and $\sim 0.33 \mathrm{~mm}$ thick crystals were observed over identical ranges of pump power. The emission from both crystals was observed in the ooe orientation, such that the efficiency of generation at low intensities is primarily determined by $d_{14}$ (see Section 3.1.2). Both crystals were pumped at $1.16 \mu \mathrm{m}(\sim 1.07 \mathrm{eV})$. Referring to the coherence length maps for GaP (Figure 2.2 (b)) and (110)-cut ZnGeP2 (Figure 4.1 (a)), both crystals show good phase matching at this pump wavelength and for crystal thickness within the detection bandwidth of $3 \mathrm{THz}$. The refractive indices of $\mathrm{GaP}$ and $\mathrm{ZnGeP}_{2}$ at $1.16 \mu \mathrm{m}$ are 3.09 and 3.21 respectively resulting in pump transmissivities of 0.74 and 0.72 . A rigid straight edge was used to assure that the $\mathrm{GaP}_{\text {and }} \mathrm{ZnGeP}_{2}$ samples were placed in identical positions in the pump beam path.

The data are shown in Figure 5.6. Error bars indicate experimental standard deviations including any error associated with switching between samples. Because both crystals are 
relatively thin, differences in linear near-infrared and $\mathrm{THz}$ absorption will only slightly affect the relative linear slopes at low power. For identical pump spot sizes in the two crystals $\left|E_{T H z}\right| \propto d_{14} P_{o}$, where $P_{o}$ is the pump power. Thus, the ratio of the slopes of the GaP and $\mathrm{ZnGeP}_{2}$ data is expected to be near to that of the ratio of literature $d_{14}$ values from Table 1:

$d_{14}^{Z n G e P_{2}} / d_{14}^{G a P}=1.82$. In fact, the data suggest that $\mathrm{ZnGeP}_{2}$ performs somewhat better than expected; the measured ratio of the slopes is 3.07. Some discrepancy between the measured and predicted ratio is expected: the assumption of constant $d_{14}$ across the near-infrared is only an approximation and the EO coefficients of the two materials were measured at significantly different wavelengths.

\subsection{Chapter Summary}

The work of this chapter comprises an extensive study of $\mathrm{ZnGeP}_{2}$ as a collinear optical rectification source of pulsed terahertz radiation. Depending on the application, $\mathrm{ZnGeP}_{2}$ may be a superior source compared to GaP or GaAs pumped in the infrared. Hence, moderate length crystals are useful for pumping by mode-locked fiber lasers in the telecom band.

There are three critical lengths that should be considered in the design of a terahertz source: First, the coherence length $L_{c}$, which defines the useful frequency range for velocity matching; second, the dispersion length $L_{D}$, which defines the significance of linear dispersioninduced broadening of the pump pulse; and third, the nonlinear length $L_{N L}$, which defines the significance of nonlinear distortion of the pump pulse by self-phase modulation. In addition to these critical lengths, the single and multiphoton absorption coefficients for the pump and the free-carrier absorption for the terahertz must be considered. From these considerations the useful envelope of the $\mathrm{THz}$ generation from $\mathrm{ZnGeP}_{2}$ is determined.

This work also demonstrates that for moderate length $\mathrm{ZnGeP}_{2}$ crystals ( $1 \mathrm{~mm}$ ) pumped with high intensity ( $15 \mathrm{GW} / \mathrm{cm} 2)$ the terahertz pulse shape and bandwidth are maintained, making this crystal suitable for wide-area excitation to produce intense pulses of terahertz radiation in the collinear pump geometry. 


\section{Chapter 6 Conclusions}

Given the ever-improving growth quality of ternary chalcopyrite semiconductors fueled by fundamental and practical interest and the continuing demand for efficient $\mathrm{THz}$ sources in compact systems, further interest in $\mathrm{ZnGeP}_{2}$ and $\mathrm{CdGeP}_{2}$ as $\mathrm{THz}$ sources is probable. This work provides an initial guide for their use in practical applications while providing insight into the physical mechanisms governing emission efficiency. Specifically, chalcopyrite structured semiconductors are considered as alternatives to commonly used zincblende semiconductors for efficient and broadband $\mathrm{THz}$ optical rectification, with emphasis on applications in THz TDS and TRTS (Section 2.4). In practice, generation efficiency is of clear importance for improving the signal-to-noise ratio in $\mathrm{THz}$ spectroscopy experiments, while broadband generation allows for spectroscopic measurements over a broader range of $\mathrm{THz}$ frequencies. Comparison of chalcopyrite $\mathrm{THz}$ sources to binary zincblende GaP and GaAs is of interest due to their efficient and broadband generation in combination with compact and cost-effective fiber laser pump sources $[36-39,45]$, as well as the similar attributes to their ternary counterparts; see Section 1.2. Fundamental and practical understanding obtained concerning $\mathrm{THz}$ optical rectification in chalcopyrite semiconductors is summarized and discussed in the final sections of Chapters 3-5.

In thin crystals, experiments show higher efficiency emission from $\mathrm{ZnGeP}_{2}$, at longer near-infrared wavelengths, in comparison to GaP due to larger nonlinear coefficients [46], while emission efficiency in GaAs is diminished in comparison to that of $\mathrm{ZnGeP}_{2}$ at high pump intensities due to the presence of 2PA below $\sim 1.75 \mu \mathrm{m}$. Mapping of the coherence lengths for $\mathrm{ZnGeP}_{2}$ (Section 4.1), GaAs, and GaP (Subsection 2.1.1.2) indicate that $\mathrm{ZnGeP}_{2}$ exhibits similar or superior potential for broad-bandwidth $\mathrm{THz}$ emission. Through tabulation of parameters

relevant to efficient $\mathrm{THz}$ generation for several chalcopyrite semiconductors and mapping of its coherence length, $\mathrm{CdGeP}_{2}$ is also identified as a promising $\mathrm{THz}$ source with a nonlinear coefficient about twice that of $\mathrm{ZnGeP}_{2}$ and broadband phase matching comparable to that of $\mathrm{ZnGeP}_{2}, \mathrm{GaP}$ and $\mathrm{GaAs}$ for thin crystals. $\mathrm{CdGeP}_{2}$ also shows superior phase matching at the fiber laser line of $1550 \mathrm{~nm}$ while avoiding 2PA, making it a potentially attractive $\mathrm{THz}$ source in the 
development of compact and cost-effective THz spectroscopy systems with good signal-tonoise and broad-bandwidth sensitivity.

For applications, both the orientation dependence of emission efficiency and scalability of $\mathrm{THz}$ emission with crystal length and pump intensity are of clear interest. Determination of the most efficient planes for optical rectification, and the rotation dependence within these planes (Chapter 3) [112], as well as the envelope of efficient and broadband emission as a function of intensity and crystal length (Chapter 5 ) should prove valuable to those interested in $\mathrm{ZnGeP}_{2}$ as a $\mathrm{THz}$ source. Modeling these data has also provided a sound understanding of the strong effects of birefringence on emission-efficiency while defining multiphoton pump depletion and resulting THz FCA as the primary contributors to a rollover in THz efficiency at high pump intensities. Deviation of the data from our model in thick crystals at high pump intensity is attributable to the detrimental effects of SPM. Because ZnGeP 2 exhibits a comparable or lower nonlinear index of refraction relative to GaP and GaAs respectively [36] the effect of SPM are expected to be of equal or greater significance in these materials for increasing pump intensity and crystal thickness. $\mathrm{ZnGeP}_{2}$ is routinely grown in large boules, making wide-area excitation [34,35] a promising option for avoidance of MPA-induced FCA and SPM.

Experimental time- and frequency-domain phase-matching dispersion maps, of Section 4.2, provide a first quasi-continuous view of the effects of phase mismatch on optical rectification $\mathrm{THz}$ transients while specifically demonstrating the range of efficient and broadband generation in $\mathrm{ZnGeP}_{2}$ for the (110)-ooe and (012)-eeo orientations.

While $\mathrm{CdGeP}_{2}$ presents itself as a particularly and potentially promising $\mathrm{THz}$ optical rectification source, more data and information are necessary to corroborate calculations of phase matched pump wavelengths and its considerably large reported electro-optic coefficients. The nonlinear index of refraction of $\mathrm{CdGeP}_{2}$ remains undefined. Based on a bandgap scaling law of [137] the nonlinear index should fall between that of $\mathrm{ZnGeP}_{2}$ and GaAs [36]. Considering that the efficiency of emission from $\mathrm{CdGeP}_{2}$ could potentially be greater than twice that from $\mathrm{ZnGeP}_{2}$, repetition of the experiments and modeling preformed in Chapter 5 for $\mathrm{CdGeP}_{2}$ is therefore strongly recommended for future investigation. Emitted $\mathrm{THz}$ electric field 
amplitudes from thin crystals of $\mathrm{GaP}, \mathrm{ZnGeP}_{2}$, and $\mathrm{CdGeP}_{2}$ in the same orientation (and of similar thickness) should also be measured for direct reference. Alternatively the generated $\mathrm{THz}$ pulse energies should be directly measured.

While the work of this dissertation has focused primarily on chalcopyrite semiconductors as $\mathrm{THz}$ sources, equal benefit can be obtained in a $\mathrm{THz}$ spectroscopy setup by applying these crystals in EO detection. This is because optical rectification and EO sampling share second-order nonlinear tensors; see Subsection 2.2.1. Also, for identical pump/probe wavelengths, the phase matching conditions are identical; thus, an efficient and broadband emitter should also be an efficient and broadband EO detector. It would be beneficial to demonstrate both $\mathrm{THz}$ emission and $\mathrm{EO}$ detection using $\mathrm{ZnGeP}_{2}$ and/or $\mathrm{CdGeP}_{2}$ and verify that the birefringence does not significantly complicate the use of these crystals in time-domain EO detection. 


\section{Bibliography}

1. S. X. Xia, C. H. Yang, G. Li, G. L. Zhu, B. Q. Yao, and Z. T. Lei, "Growth of high quality non-linear optical crystal zinc germanium phosphide for mid-infrared optical parametric oscillator," Laser Phys. 21, 1366-1370 (2011).

2. K. T. Zawilski, P. G. Schunemann, S. D. Setzler, and T. M. Pollak, "Large aperture single crystal ZnGeP 2 for high-energy applications," Journal of Crystal Growth 310, 1891-1896 (2008).

3. J. Lloyd-Hughes and T.-I. Jeon, "A review of the terahertz conductivity of bulk and nanomaterials," J. Infrared Millim. Te. 33, 871-925 (2012).

4. M. Hangyo, M. Tani, and T. Nagashima, "Terahertz time-domain spectroscopy of solids: a review," Int. J. Infrared Milli. 26, 1661-1690 (2005).

5. R. Ulbricht, E. Hendry, J. Shan, T. F. Heinz, and M. Bonn, "Carrier dynamics in semiconductors studied with time-resolved terahertz spectroscopy," Rev. Mod. Phys. 83, 543-586 (2011).

6. C. A. Schmuttenmaer, "Exploring dynamics in the far-infrared with terahertz spectroscopy," Chem. Rev. 104, 1759-1780 (2004).

7. T. Kampfrath, A. Sell, G. Klatt, A. Pashkin, S. Mährlein, T. Dekorsy, M. Wolf, M. Fiebig, A. Leitenstorfer, and R. Huber, "Coherent terahertz control of antiferromagnetic spin waves," Nat. Photon. 5, 31-34 (2011).

8. Z. D. Taylor, R. S. Singh, D. B. Bennett, P. Tewari, C. P. Kealey, N. Bajwa, M. O. Culjat, A. Stojadinovic, H. Lee, J.-P. Hubschman, E. R. Brown, and W. S. Grundfest, "THz medical imaging: in vivo hydration sensing," IEEE Trans. Terahertz Sci. 1, 201 -219 (2011).

9. J. Federici and L. Moeller, "Review of terahertz and subterahertz wireless communications," J. Appl. Phys. 107, 111101 (2010).

10. M. Tonouchi, "Cutting-edge terahertz technology," Nat. Photon. 1, 97-105 (2007).

11. H.-W. Hübers, "Terahertz technology: Towards THz integrated photonics," Nat. Photon. 4, 503504 (2010).

12. F. Blanchard, G. Sharma, L. Razzari, X. Ropagnol, H.-C. Bandulet, F. Vidal, R. Morandotti, J.-C. Kieffer, T. Ozaki, H. Tiedje, H. Haugen, M. Reid, and F. Hegmann, "Generation of intense terahertz radiation via optical methods," IEEE J. Sel. Top. Quant. Electron. 17, 5 -16 (2011).

13. M. C. Hoffmann and J. A. Fülöp, "Intense ultrashort terahertz pulses: generation and applications," J. Phys. D: Appl. Phys. 44, 1-13 (2011).

14. M. F. Kimmitt, "Restrahlen to T-rays -100 years of terahertz radiation," J. of Biol. Phys. 29, 77-85 (2003).

15. S. Jayaraman and C. H. Lee, "Observation of two-photon conductivity in GaAs with nanosecond and picosecond light pulses," Appl. Phys. Lett. 20, 392-395 (1972).

16. C. H. Lee, "Picosecond optoelectronic switching in GaAs," Appl. Phys. Lett. 30, 84-86 (1977).

17. G. Mourou, C. V. Stancampiano, A. Antonetti, and A. Orszag, "Picosecond microwave pulses generated with a subpicosecond laser-driven semiconductor switch," Appl. Phys. Lett. 39, 295296 (1981).

18. D. H. Auston, K. P. Cheung, and P. R. Smith, "Picosecond photoconducting Hertzian dipoles," Appl. Phys. Lett. 45, 284-286 (1984).

19. D. H. Auston, K. P. Cheung, J. A. Valdmanis, and D. A. Kleinman, "Cherenkov radiation from femtosecond optical pulses in electro-optic media," Phys. Rev. Lett. 53, 1555-1558 (1984).

20. P. R. Smith, D. H. Auston, and M. C. Nuss, "Subpicosecond photoconducting dipole antennas," IEEE J. Quant. Electron. 24, 255 -260 (1988). 
21. C. Fattinger and D. Grischkowsky, "Terahertz beams," Appl. Phys. Lett. 54, 490-492 (1989).

22. M. van Exter and D. Grischkowsky, "Carrier dynamics of electrons and holes in moderately doped silicon," Phys. Rev. B 41, 12140-12149 (1990).

23. M. C. Nuss, K. W. Goossen, J. P. Gordon, P. M. Mankiewich, M. L. O'Malley, and M. Bhushan, "Terahertz time-domain measurement of the conductivity and superconducting band gap in niobium," J. Appl. Phys. 70, 2238-2241 (1991).

24. G. Arjavalingam, N. Theophilou, Y. Pastol, G. V. Kopcsay, and M. Angelopoulos, "Anisotropic conductivity in stretch-oriented polymers measured with coherent microwave transient spectroscopy," J. Chem. Phys. 93, 6-9 (1990).

25. L. Xu, X.-C. Zhang, and D. H. Auston, "Terahertz beam generation by femtosecond optical pulses in electro-optic materials," Appl. Phys. Lett. 61, 1784-1786 (1992).

26. X.-C. Zhang, Y. Jin, and X. F. Ma, "Coherent measurement of THz optical rectification from electrooptic crystals," Appl. Phys. Lett. 61, 2764-2766 (1992).

27. X.-C. Zhang, X. F. Ma, Y. Jin, T.-M. Lu, E. P. Boden, P. D. Phelps, K. R. Stewart, and C. P. Yakymyshyn, "Terahertz optical rectification from a nonlinear organic crystal," Appl. Phys. Lett. 61, 3080-3082 (1992).

28. E. B. Petersen, W. Shi, A. Chavez-Pirson, N. Peyghambarian, and A. T. Cooney, "Efficient parametric terahertz generation in quasi-phase-matched GaP through cavity enhanced difference-frequency generation," Appl. Phys. Lett. 98, 121119-121119-3 (2011).

29. L. Hou and W. Shi, "An LT-GaAs terahertz photoconductive antenna with high emission power, low noise, and good stability," IEEE T. Electron Dev. 60, 1619-1624 (2013).

30. J. Dai, J. Liu, and X.-C. Zhang, "Terahertz wave air photonics: terahertz wave generation and detection with laser-induced gas plasma," IEEE J. Sel. Top. Quant. Electron. 17, 183 -190 (2011).

31. J. A. Fülöp, L. Pálfalvi, G. Almási, and J. Hebling, "Design of high-energy terahertz sources based on optical rectification," Opt. Express 18, 12311-12327 (2010).

32. M. C. Hoffmann, K.-L. Yeh, J. Hebling, and K. A. Nelson, "Efficient terahertz generation by optical rectification at $1035 \mathrm{~nm}$," Opt. Express 15, 11706-11713 (2007).

33. M. I. Bakunov, S. B. Bodrov, and E. A. Mashkovich, "Terahertz generation with tilted-front laser pulses: dynamic theory for low-absorbing crystals," J. Opt. Soc. Am. B 28, 1724 (2011).

34. F. Blanchard, L. Razzari, H. C. Bandulet, G. Sharma, R. Morandotti, J. C. Kieffer, T. Ozaki, M. Reid, H. F. Tiedje, H. K. Haugen, and F. A. Hegmann, "Generation of $1.5 \mu \mathrm{J}$ single-cycle terahertz pulses by optical rectification from a large aperture ZnTe crystal," Opt. Express 15, 13212-13220 (2007).

35. T. Löffler, T. Hahn, M. Thomson, F. Jacob, and H. Roskos, "Large-area electro-optic ZnTe terahertz emitters," Opt. Express 13, 5353-5362 (2005).

36. K. I. Vodopyanov, "Optical THz-wave generation with periodically-inverted GaAs," Laser Photon. Rev. 2, 11-25 (2008).

37. J.-P. Negel, R. Hegenbarth, A. Steinmann, B. Metzger, F. Hoos, and H. Giessen, "Compact and cost-effective scheme for $\mathrm{THz}$ generation via optical rectification in $\mathrm{GaP}$ and $\mathrm{GaAs}$ using novel fs laser oscillators," Appl. Phys. B 103, 45-50 (2011).

38. M. Nagai, K. Tanaka, H. Ohtake, T. Bessho, T. Sugiura, T. Hirosumi, and M. Yoshida, "Generation and detection of terahertz radiation by electro-optical process in GaAs using $1.56 \mu \mathrm{m}$ fiber laser pulses," Appl. Phys. Lett. 85, 3974-3976 (2004).

39. G. Chang, C. J. Divin, C.-H. Liu, S. L. Williamson, A. Galvanauskas, and T. B. Norris, "Power scalable compact THz system based on an ultrafast Yb-doped fiber amplifier," Opt. Express 14, 7909 (2006).

40. I. Tomita, H. Suzuki, H. Ito, H. Takenouchi, K. Ajito, R. Rungsawang, and Y. Ueno, "Terahertz-wave generation from quasi-phase-matched GaP for 1.55 um pumping," Appl. Phys. Lett. 88, 071118071118-3 (2006). 
41. J. Darmo, M. Martl, and K. Unterrainer, "Quasi phase-matched terahertz detector," Electron. Lett. 46, $788-790$ (2010).

42. L. Gordon, G. L. Woods, R. C. Eckardt, R. R. Route, R. S. Feigelson, M. M. Fejer, and R. Byer, "Diffusion-bonded stacked GaAs for quasiphase-matched second-harmonic generation of a carbon dioxide laser," Electron. Lett. 29, 1942 -1944 (1993).

43. L. A. Eyres, P. J. Tourreau, T. J. Pinguet, C. B. Ebert, J. S. Harris, M. M. Fejer, L. Becouarn, B. Gerard, and E. Lallier, "All-epitaxial fabrication of thick, orientation-patterned GaAs films for nonlinear optical frequency conversion," Appl. Phys. Lett. 79, 904-906 (2001).

44. Y.-S. Lee, W. C. Hurlbut, K. L. Vodopyanov, M. M. Fejer, and V. G. Kozlov, "Generation of multicycle terahertz pulses via optical rectification in periodically inverted GaAs structures," Appl. Phys. Lett. 89, 181104-181104-3 (2006).

45. G. Imeshev, M. E. Fermann, K. L. Vodopyanov, M. M. Fejer, X. Yu, J. S. Harris, D. Bliss, and C. Lynch, "High-power source of THz radiation based on orientation-patterned GaAs pumped by a fiber laser," Opt. Express 14, 4439-4444 (2006).

46. J. D. Rowley, J. K. Pierce, A. T. Brant, L. E. Halliburton, N. C. Giles, P. G. Schunemann, and A. D. Bristow, "Broadband terahertz pulse emission from ZnGeP2," Opt. Lett. 37, 788-790 (2012).

47. J. L. Shay and J. H. Wernick, Ternary Chalcopyrite Semiconductors: Growth, Electronic Properties, and Applications (Pergamon Press, 1975).

48. A. A. Rockett, "Current status and opportunities in chalcopyrite solar cells," Current Opinion in Solid State and Materials Science 14, 143-148 (2010).

49. K. T. Zawilski, P. G. Schunemann, T. C. Pollak, D. E. Zelmon, N. C. Fernelius, and F. Kenneth Hopkins, "Growth and characterization of large $\mathrm{CdSiP}_{2}$ single crystals," J. Cryst. Growth 312, 1127-1132 (2010).

50. G. A. Medvedkin and V. G. Voevodin, "Magnetic and optical phenomena in nonlinear optical crystals ZnGeP 2 and $\mathrm{CdGeP}_{2}$," J. Opt. Soc. Am. B 22, 1884-1898 (2005).

51. S. C. Erwin and I. Žutić, "Tailoring ferromagnetic chalcopyrites," Nat. Mater. 3, 410-414 (2004).

52. V. M. Novotortsev, A. V. Kochura, and S. F. Marenkin, "New ferromagnetics based on manganesealloyed chalcopyrites $A^{11} B^{I V} C_{2}^{V}$," Inorg. Mater. 46, 1421-1436 (2010).

53. W. Feng, D. Xiao, J. Ding, and Y. Yao, "Three-dimensional topological insulators in I-III- $\mathrm{VI}_{2}$ and IIIV-V 2 chalcopyrite semiconductors," Phys. Rev. Lett. 106, 016402 (2011).

54. M. Grundmann, The Physics of Semiconductors: An Introduction Including Nanophysics and Applications (Springer, 2011).

55. G. D. Boyd, E. Buehler, F. Storz, and J. Wernick, "Linear and nonlinear optical properties of ternary $A^{\prime \prime} B^{1 V} C_{2}{ }^{V}$ chalcopyrite semiconductors," IEEE J. Quant. Electron. 8, 419-426 (1972).

56. E. D. Palik, Handbook of Optical Constants of Solids (Academic Press, 1998).

57. P. G. Schunemann and T. M. Pollak, "Ultralow gradient HGF-grown $\mathrm{ZnGeP}_{2}$ and $\mathrm{CdGeAs}_{2}$ and their optical properties," MRS Bull. 23, 23-27 (1998).

58. G. A. Verozubova, A. O. Okunev, A. I. Gribenyukov, A. Y. Trofimiv, E. M. Trukhanov, and A. V. Kolesnikov, "Growth and defect structure of $\mathrm{ZnGeP}_{2}$ crystals," J. Cryst. Growth 312, 1122-1126 (2010).

59. K. L. Vodopyanov, F. Ganikhanov, J. P. Maffetone, I. Zwieback, and W. Ruderman, "ZnGeP 2 optical parametric oscillator with 3.8-12.4 $\mu$ m tunability," Opt. Lett. 25, 841-843 (2000).

60. M. W. Todd, R. A. Provencal, T. G. Owano, B. A. Paldus, A. Kachanov, K. L. Vodopyanov, M. Hunter, S. L. Coy, J. I. Steinfeld, and J. T. Arnold, "Application of mid-infrared cavity-ringdown spectroscopy to trace explosives vapor detection using a broadly tunable $(6-8 \mu \mathrm{m})$ optical parametric oscillator," Appl. Phys. B: Lasers O 75, 367-376 (2002).

61. K. L. Vodopyanov and P. G. Schunemann, "Broadly tunable noncritically phase-matched $\mathrm{ZnGeP}_{2}$ optical parametric oscillator with a $2 \mu \mathrm{J}$ pump threshold," Opt. Lett. 28, 441-443 (2003). 
62. W. Shi, Y. J. Ding, and P. G. Schunemann, "Coherent terahertz waves based on differencefrequency generation in an annealed zinc-germanium phosphide crystal: improvements on tuning ranges and peak powers," Opt. Commun. 233, 183-189 (2004).

63. Y. J. Ding and W. Shi, "Widely tunable monochromatic $\mathrm{THz}$ sources based on phase-matched difference-frequency generation in nonlinear-optical crystals: A novel approach," Laser Phys. 16, 562-570 (2006).

64. S. Xia, M. Wang, C. Yang, Z. Lei, G. Zhu, and B. Yao, "Vertical Bridgman growth and characterization of large ZnGeP 2 single crystals," J. Cryst. Growth 314, 306-309 (2011).

65. G. Zhang, X. Tao, S. Wang, Q. Shi, H. Ruan, and L. Chen, "Growth improvement and quality evaluation of $\mathrm{ZnGeP}_{2}$ single crystals using vertical Bridgman method," J. Cryst. Growth 352, 67-71 (2012).

66. G. A. Verozubova, A. I. Gribenyukov, V. V. Korotkova, and M. P. Ruzaikin, "ZnGeP 2 synthesis and growth from melt," Mat. Sci. Eng. B 48, 191-197 (1997).

67. B. R. Johnson, K. T. Zawilski, P. G. Schunemann, P. R. Staver, T. M. Pollak, and E. P. Chickilis, "Investigation of birefringence uniformity of mid-IR nonlinear optical crystals," in Nonlinear Frequency Generation and Conversion: Materials, Devices, and Applications XI (2012), pp. 824016-824016.

68. M. Mineo and C. Paoloni, "Comparison of THz backward wave oscillators based on corrugated waveguides," PIER Lett. 30, 163-171 (2012).

69. B. S. Williams, "Terahertz quantum-cascade lasers," Nat. Photon. 1, 517-525 (2007).

70. P. Tan, J. Huang, K. Liu, Y. Xiong, and M. Fan, "Terahertz radiation sources based on free electron lasers and their applications," Sci. China Inf. Sci. 55, 1-15 (2012).

71. J. Barros, C. Evain, L. Manceron, J.-B. Brubach, M.-A. Tordeux, P. Brunelle, L. Nadolski, A. Loulergue, M.-E. Couprie, S. Bielawski, C. Szwaj, and P. Roy, "Coherent synchrotron radiation for broadband terahertz spectroscopy," Rev. Sci. Instrum. 84, 033102-033102-4 (2013).

72. K. Reimann, "Table-top sources of ultrashort THz pulses," Rep. Prog. Phys. 70, 1597 (2007).

73. Y.-S. Lee, Principles of Terahertz Science and Technology (Springer, 2008).

74. R. W. Boyd, Nonlinear Optics (Acad. Press, 2003).

75. Y. R. Shen, The Principles of Nonlinear Optics (Wiley-Interscience, 2003).

76. P. Gu and M. Tani, "Terahertz radiation from semiconductor surfaces," in Terahertz Optoelectronics, K. Sakai, ed., Top. Appl. Phys. No. 97 (Springer Berlin Heidelberg, 2005), pp. 6398.

77. Y. H. Jin and X.-C. Zhang, "Terahertz Optical Rectification," J. Nonlinear Opt. Phys. 04, 459-495 (1995).

78. J. Faure, J. V. Tilborg, R. A. Kaindl, and W. P. Leemans, "Modelling laser-based table-top THz sources: optical rectification, propagation and electro-optic sampling," Opt. Quant. Electron. 36, 681-697 (2004).

79. G. D. Boyd, T. J. Bridges, M. A. Pollack, and E. H. Turner, "Microwave nonlinear susceptibilities due to electronic and ionic anharmonicities in acentric crystals," Phys. Rev. Lett. 26, 387-390 (1971).

80. R. L. Sutherland, D. G. McLean, and S. Kirkpatrick, Handbook of Nonlinear Optics (CRC Press, 2003).

81. A. Nahata, A. S. Weling, and T. F. Heinz, "A wideband coherent terahertz spectroscopy system using optical rectification and electro-optic sampling," Appl. Phys. Lett. 69, 2321 (1996).

82. A. S. Barker, "Dielectric dispersion and phonon line shape in gallium phosphide," Phys. Rev. 165, 917-922 (1968).

83. F. L. Madarasz, J. O. Dimmock, N. Dietz, and K. J. Bachmann, "Sellmeier parameters for ZnGaP and GaP," Journal of Applied Physics 87, 1564-1565 (2000). 
84. T. Skauli, P. S. Kuo, K. L. Vodopyanov, T. J. Pinguet, O. Levi, L. A. Eyres, J. S. Harris, M. M. Fejer, B. Gerard, L. Becouarn, and E. Lallier, "Improved dispersion relations for GaAs and applications to nonlinear optics," Journal of Applied Physics 94, 6447-6455 (2003).

85. W. J. Moore and R. T. Holm, "Infrared dielectric constant of gallium arsenide," Journal of Applied Physics 80, 6939-6942 (1996).

86. Q. Wu and X.-C. Zhang, "7 terahertz broadband GaP electro-optic sensor," Appl. Phys. Lett. 70, 1784-1786 (1997).

87. M. Rumi and J. W. Perry, "Two-photon absorption: an overview of measurements and principles," Adv. Opt. Photon. 2, 451-518 (2010).

88. R. R. Birge, "2 - One-photon and two-photon excitation spectroscopy," in Ultrasensitive Laser Spectroscopy, David Kliger, ed. (Academic Press, 1983), pp. 109-174.

89. S.-G. Park, M. R. Melloch, and A. M. Weiner, "Comparison of terahertz waveforms measured by electro-optic and photoconductive sampling," Appl. Phys. Lett. 73, 3184-3186 (1998).

90. S.-G. Park, M. R. Melloch, and A. M. Weiner, "Analysis of terahertz waveforms measured by photoconductive and electrooptic sampling," IEEE J. Quant. Electron. 35, 810-819 (1999).

91. Y. Cai, I. Brener, J. Lopata, J. Wynn, L. Pfeiffer, J. B. Stark, Q. Wu, X. C. Zhang, and J. F. Federici, "Coherent terahertz radiation detection: Direct comparison between free-space electro-optic sampling and antenna detection," Appl. Phys. Lett. 73, 444-446 (1998).

92. P. C. M. Planken, H.-K. Nienhuys, H. J. Bakker, and T. Wenckebach, "Measurement and calculation of the orientation dependence of terahertz pulse detection in ZnTe," J. Opt. Soc. Am. B 18, 313317 (2001).

93. I. Jovanovic, "Chirped-Pulse Amplification," Optik \& Photonik 5, 30-33 (2010).

94. D. R. Skinner and R. E. Whitcher, "Measurement of the radius of a high-power laser beam near the focus of a lens," J. Phys. E: Sci. Instrum. 5, 237 (1972).

95. J. Dai, J. Zhang, W. Zhang, and D. Grischkowsky, "Terahertz time-domain spectroscopy characterization of the far-infrared absorption and index of refraction of high-resistivity, floatzone silicon," J. Opt. Soc. Am. B 21, 1379-1386 (2004).

96. F. Salin, P. Georges, G. Roger, and A. Brun, "Single-shot measurement of a 52-fs pulse," Appl. Opt. 26, 4528-4531 (1987).

97. V. V. Voitsekhovskii, A. A. Volkov, G. A. Komandin, and Y. A. Shakir, "Dielectric properties of ZnGeP 2 in the far infrared," Phys. Solid State 37, 1198-1199 (1995).

98. L. Duvillaret, F. Garet, and J.-L. Coutaz, "Highly precise determination of optical constants and sample thickness in terahertz time-domain spectroscopy," Appl. Opt. 38, 409-415 (1999).

99. M. Krüger, S. Funkner, E. Bründermann, and M. Havenith, "Uncertainty and ambiguity in terahertz parameter extraction and data analysis," J. Infrared Millim. Te. 32, 699-715 (2011).

100. M. C. Beard, G. M. Turner, J. E. Murphy, O. I. Micic, M. C. Hanna, A. J. Nozik, and C. A. Schmuttenmaer, "Electronic coupling in InP nanoparticle arrays," Nano Lett. 3, 1695-1699 (2003).

101. E. Cánovas, P. Moll, S. A. Jensen, Y. Gao, A. J. Houtepen, L. D. A. Siebbeles, S. Kinge, and M. Bonn, "Size-dependent electron transfer from $\mathrm{PbSe}$ quantum dots to $\mathrm{SnO}_{2}$ monitored by picosecond terahertz spectroscopy," Nano Lett. 11, 5234-5239 (2011).

102. F. Meng, J. Li, S. K. Cushing, J. Bright, M. Zhi, J. D. Rowley, Z. Hong, A. Manivannan, A. D. Bristow, and $\mathrm{N}$. Wu, "Photocatalytic water oxidation by hematite/reduced graphene oxide composites," ACS Catal. 3, 746-751 (2013).

103. S. Kaniyankandy, S. N. Achary, S. Rawalekar, and H. N. Ghosh, "Ultrafast relaxation dynamics in graphene oxide: evidence of electron trapping," J. Phys. Chem. C 115, 19110-19116 (2011).

104. A. G. Joly, J. R. Williams, S. A. Chambers, G. Xiong, W. P. Hess, and D. M. Laman, "Carrier dynamics in $\alpha-\mathrm{Fe}_{2} \mathrm{O}_{3}(0001)$ thin films and single crystals probed by femtosecond transient absorption and reflectivity," J. Appl. Phys. 99, 053521-053521-6 (2006). 
105. J. Li, S. K. Cushing, J. Bright, F. Meng, T. R. Senty, P. Zheng, A. D. Bristow, and N. Wu, "Ag@Cu, O core-shell nanoparticles as visible-light plasmonic photocatalysts," ACS Catal. 3, 47-51 (2013).

106. J. Hebling, K.-L. Yeh, M. C. Hoffmann, B. Bartal, and K. A. Nelson, "Generation of high-power terahertz pulses by tilted-pulse-front excitation and their application possibilities," J. Opt. Soc. Am. B 25, B6-B19 (2008).

107. C.-W. Chen, T.-T. Tang, S.-H. Lin, J. Y. Huang, C.-S. Chang, P.-K. Chung, S.-T. Yen, and C.-L. Pan, "Optical properties and potential applications of $\varepsilon$-GaSe at terahertz frequencies," J. Opt. Soc. Am. B 26, A58-A65 (2009).

108. A. Schneider, M. Neis, M. Stillhart, B. Ruiz, R. U. A. Khan, and P. Günter, "Generation of terahertz pulses through optical rectification in organic DAST crystals: theory and experiment," J. Opt. Soc. Am. B 23, 1822-1835 (2006).

109. F. D. Brunner, O.-P. Kwon, S.-J. Kwon, M. Jazbinsek, A. Schneider, and P. Günter, "A hydrogenbonded organic nonlinear optical crystal for high-efficiency terahertz generation and detection," Opt. Express 16, 16496-16508 (2008).

110. M. H. Rakowsky, W. K. Kuhn, W. J. Lauderdale, L. E. Halliburton, G. J. Edwards, M. P. Scripsick, P. G. Schunemann, T. M. Pollak, M. C. Ohmer, and F. K. Hopkins, "Electron paramagnetic resonance study of a native acceptor in as-grown ZnGeP," Appl. Phys. Lett. 64, 1615-1617 (1994).

111. C. Lee and R. A. Lewis, "Optical rectification for terahertz generation," Phys. Status Solidi 8, 27612765 (2011).

112. J. D. Rowley, J. K. Wahlstrand, K. T. Zawilski, P. G. Schunemann, N. C. Giles, and A. D. Bristow, "Terahertz generation by optical rectification in uniaxial birefringent crystals," Opt. Express 20, 16968-16973 (2012).

113. G. Kanellis and K. Kampas, "Phonons optiques par réflexion infrarouge des composés ternaires $\mathrm{AgGaSe}_{2}$ et $\mathrm{AgGaTe}_{2}$," Journal de physique 38, 833 - 839 (1977).

114. G. D. Holah, J. S. Webb, and H. Montgomery, "Lattice dynamics of AgGaS 2 ," J. Phys. C: Solid State Phys. 7, 3875 (1974).

115. Nonlinear Optical Crystals: A Complete Survey (Springer, 2005).

116. A. Miller, G. D. Holah, and W. C. Clark, "Infrared dielectric dispersion of $\mathrm{ZnGeP}_{2}$ and $\mathrm{CdGeP}_{2}$," J. Phys. Chem. Solids 35, 685-693 (1974).

117. G. C. Bhar and G. Ghosh, "Temperature-dependent Sellmeier coefficients and coherence lengths for some chalcopyrite crystals," J. Opt. Soc. Am. 69, 730-733 (1979).

118. G. C. Bhar, "Refractive index interpolation in phase-matching," Appl. Opt. 15, 305_1-307 (1976).

119. M. J. Weber, Handbook of Optical Materials (CRC Press, 2010).

120. P. Vohl, "Synthesis and crystal growth of CdGeP," J. Electron. Mater. 8, 517-528 (1979).

121. E. H. Turner, E. Buehler, and H. Kasper, "Electro-optic behavior and dielectric constants of $\mathrm{ZnGeP}_{2}$ and $\mathrm{CuGaS}_{2}$," Phys. Rev. B 9, 558-561 (1974).

122. V. M. Cound, P. H. Davies, K. F. Hulme, and D. Robertson, "The electro-optic coefficients of silver thiogallate $\left(\mathrm{AgGaS}_{2}\right)$," J. Phys. C: Solid State Phys. 3, L83 (1970).

123. H. Horinaka, H. Sonomura, and T. Miyauchi, "Linear electro-optic effect of AgGaSe," Jpn. J. Appl. Phys. 21, 1485-1488 (1982).

124. O. Madelung, Semiconductors: Data Handbook (Springer Verlag, 2004).

125. M. Bettini and A. Miller, "Optical phonons in $\mathrm{ZnGeP}_{2}$ and $\mathrm{CdGeP}_{2}$," Phys. Status Solidi 66, 579-586 (1974).

126. L. Artús, J. Pascual, J. Pujol, J. Camassel, and R. S. Feigelson, "Vibrational properties of CdGeP2," Phys. Rev. B 43, 2088-2096 (1991).

127. I.-H. Choi and P. Y. Yu, "Pressure dependence of the natural birefringence in the II-IV-V 2 chalcopyrite CdGeP2," Phys. Status Solidi 235, 307-311 (2003). 
128. V. M. Novotortsev, S. A. Varnavskii, S. F. Marenkin, L. I. Koroleva, R. V. Demin, V. M. Trukhan, S. O. Klimonskii, and V. D. Kuznetsov, "Ferromagnetic material $\mathrm{CdGeP}_{2}: M n$ for spintronics," Russ. J. Inorg. Chem. 51, 1153-1156 (2006).

129. P. Mahadevan and A. Zunger, "Room-Temperature Ferromagnetism in Mn-Doped Semiconducting CdGeP2," Phys. Rev. Lett. 88, 047205 (2002).

130. D. Li, X. Qi, and S. Liu, "A theoretical analysis of optical-to-THz conversion efficiency via optical rectification," Sci. China Ser. E-Technol. Sci. 51, 2080-2088 (2008).

131. K. Wynne and J. J. Carey, "An integrated description of terahertz generation through optical rectification, charge transfer, and current surge," Opt. Commun. 256, 400-413 (2005).

132. N. C. J. van der Valk, P. C. M. Planken, A. N. Buijserd, and H. J. Bakker, "Influence of pump wavelength and crystal length on the phase matching of optical rectification," J. Opt. Soc. Am. B 22, 1714-1718 (2005).

133. S. M. Harrel, R. L. Milot, J. M. Schleicher, and C. A. Schmuttenmaer, "Influence of free-carrier absorption on terahertz generation from ZnTe (110)," J. Appl. Phys. 107, 033526-033526-5 (2010).

134. B. Gu, J. He, W. Ji, and H.-T. Wang, "Three-photon absorption saturation in ZnO and ZnS crystals," J. Appl. Phys. 103, 073105-073105-5 (2008).

135. R. H. Stolen and C. Lin, "Self-phase-modulation in silica optical fibers," Phys. Rev. A 17, 14481453 (1978).

136. G. P. Agrawal, Lightwave Technology: Telecommunication Systems (John Wiley \& Sons, 2005).

137. R. DeSalvo, A. A. Said, D. J. Hagan, E. W. Van Stryland, and M. Sheik-Bahae, "Infrared to ultraviolet measurements of two-photon absorption and $\mathrm{n}_{2}$ in wide bandgap solids," IEEE J. Quant. Electron. 32, 1324-1333 (1996). 